University of Rhode Island

DigitalCommons@URI

Open Access Master's Theses

1977

\title{
Word Meanings in Semantic Memory: Separating, Defining and Characteristic Features
}

Ageliki Nicolopoulou

University of Rhode Island

Follow this and additional works at: https://digitalcommons.uri.edu/theses

\section{Recommended Citation}

Nicolopoulou, Ageliki, "Word Meanings in Semantic Memory: Separating, Defining and Characteristic Features" (1977). Open Access Master's Theses. Paper 1631.

https://digitalcommons.uri.edu/theses/1631

This Thesis is brought to you for free and open access by DigitalCommons@URI. It has been accepted for inclusion in Open Access Master's Theses by an authorized administrator of DigitalCommons@URI. For more information, please contact digitalcommons-group@uri.edu. 
WORD MEANINGS IN SEMANTIC MEMORY:

SEPARATING, DEFINING AND

CHARACTERISTIC FEATURES

BY

AGELIKI NICOLOPOULOU

A THESIS SUBMITTED IN PARTIAL FULFILLMENT OF THE REQUIREMENTS FOR THE DEGREE OF

MASTER OF ARTS

IN

PSYCHOLOGY

UNIVERSITY OF RHODE ISLAND

1977 


\section{ABSTRACT}

Three experiments were conducted to obtain some empirical generalizations about the structure of semantic memory. In the first experiment subjects were asked to give features for five category names as well as four instances per category. Instances varied on degree of relatedness to the category. In the second experiment subjects were asked to separate the features that they had given into (a) those that were essential for the meaning of a word (defining), and (b) those that were not absolutely necessary for the word (characteristic). In the third experiment a new set of subjects were given the same five category names and 2 instances out of the previous four, each word followed by a common pool of features that were generated by its category and instances in experiment 1, and were asked to separate these features into essential, non-essential and not a property for each stimulus word. The predictions tested were that (1) subjects would produce a greater number of defining than characteristic features, (2) the first feature generated would be more frequently a defining than a characteristic feature, (3) defining features of the category would be fewer in number than defining features of the instances, and (4) the degree of relationship between the defining and characteristic features of the instance and the defining and characteristic features of its category would vary with the degree of relatedness of the instance to the category. Prediction 1 was not supported. Rather, it was found that characteristic features were more prevalent than defining features 
for both categories and instances. Prediction 2 was confirmed: there were more defining than characteristic features given as first response. Prediction 3 did not receive any support. Prediction 4 was confirmed for defining features, where it was found that decreasing levels of relatedness lowered the overlap between the instance and its category; however, the relationship between the characteristic features of the instance and its category remained at about the same high level at all degrees of relatedness.

These findings, along with the fact that the categorization of features for instance words was not altered when $\underline{S} s$ were given the category names ${ }^{1}$ features first, argue against the view that category names function as superordinates for instance in semantic memory and in favor of the view that natural language categories possess an analog structure and that category names act as "best exemplars" of each category. 


\section{ACKNOWLEDGMENT}

I would like to express my deep gratitude to those individuals who contributed substantially to the completion of this work. My thanks to Dr. Albert Silverstein, my major professor, who patiently guided this work. Through his willingness to explore areas new to him, he contributed greatly to my intellectual development. I would like to thank Dr. Peter Merenda and Dr. Wayne Velicer for their generous statistical consultation and the staff of the U.R.I. computer center for their assistance in the data analyses.

I owe special thanks to Dr. Richard Millward of Brown University, a truly generous person, who spent many hours consulting with me on difficult and crucial issues of my research. On the occasions when the project seemed overwhelming, it was he, above al1, who rekindled my enthusiasm.

I would also like to thank my officemate and dear friend Jeanne Lemkau for her contribution to my semantic memory and her moral support during endless hours at Chafee. I would finally like to thank Apostolos, Zoe, Helen and all of my family for their love and support always.

To my mother and to the memory of my father I dedicate this work. 
TABLE OF CONTENTS

Page

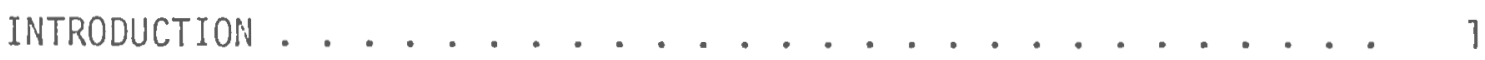

EXPERIMENT 1 ............................... 17

METHOD ............................ 17

Subjects .................. 17

Stimuli................... 18

Procedure ................ 19

RESULTS .................................. 19

EXPERIMENT 2.......................... 47

METHOD ............................. 48

Subjects ....................... 48

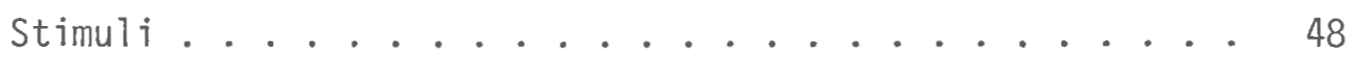

Procedure .................... 48

RESULTS ............................... 49

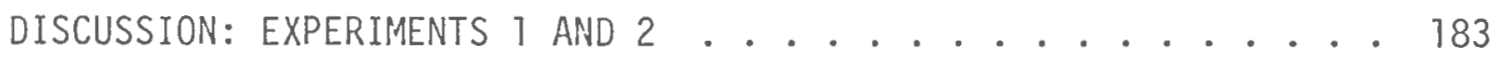

EXPERIMENT $3 \ldots \ldots$. . . . . . . . . . . . . . 187

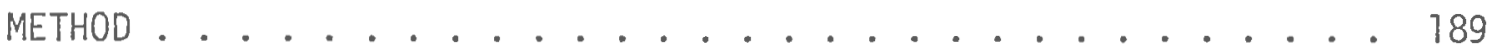

Subjects .................... 189

Stimuli ..................... 189

Procedure ................. 190

RESULTS ........................... 190

DISCUSSION: EXPERIMENT $3 \ldots \ldots$. . . . . . . . . . 193 
GENERAL DISCUSSION ..................... 196

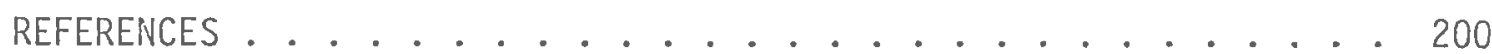

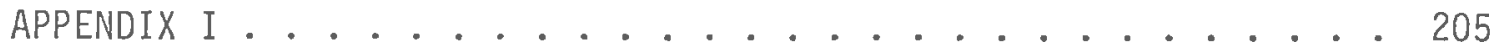
APPENDIX II ................... 223 BIBLIOGRAPHY ................... 224 


\section{LIST OF TABLES}

Table

Page

1. Frequencies and Percentages of Features from Production Memory . . . . . . . . . . . . . . . . . . .

2. Frequencies and Percentages of Essential and Non-Essential Features from Production Memory . . . . . . . . . . .

3. Means and Standard Deviations of Defining and Characteristic Features for Categories

4. Means and Standard Deviations of Defining and Characteristic Features for Instances

5. Means and Standard Deviations of Features for Instances and Categories... . . . . . . . . . . . .

6. Means and Standard Deviations of Defining and Characteristic Features for Categories and Instances . . . . . . .

7. Summary Table of Analysis of Variance for Number of Defining and Characteristic Features for Categories and Instances

8. Newman-Keuls Test of Features for Categories . . . . . 86

9. Newman-Keuls Test at Features for Instances . . . . . . 87

10. Summary Table for Partitioning of Features $X$ Categories Interaction

11. Summary Table for Partitioning of Features $X$ Instances Interaction

12. Newman-Keuls Test for Defining Features at Instances...

13. Summary Table for Partitioning of Features $X$ Categories $X$ Instances Interaction . . . . . . . . . . . 93

14. Percentages of Features from Recognition Memory . . . . 96

15. Newman-Keuls Test for Instances at $F_{1} C_{1}$. . . . . . 177

16. Newman-Keuls Test for Instances at $\mathrm{F}_{7} \mathrm{C}_{2}$. . . . . . 178 
17. Newman-Keuls Test for Instances at $F_{7} C_{4}$. . . . . . 179

18. Newman-Keuls Test for Instances at $F_{7} C_{5}$. . . . . . 180

19. Defining, Characteristic and Other Features Given as First Response for Categories and Instances . . . . . 187

20. Comparison of Defining, Characteristic and Other Features Given as the First Feature for Average of Instances and Category Names.............. 181

21. Comparison of Defining, Characteristic and 0ther Features Given as First Response for Levels of Relatedness and

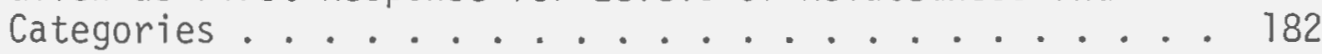

22. Chi-squares and Contingency Coefficients on the Number of Essential, Non-Essential and Not a Property Features Shared by a Category Name and its Instances . . . . . 192

23. Essential and Non-Essential Classes of Features for Category and Levels of Relatedness... . . . . . . 194 


\section{LIST OF FIGURES}

Figure

Page

1. Mean Number of Defining Features for Category Name and Levels of Relatedness for Categories . . . . . . .

2. Mean Number of Characteristic Features for Category

Name and Levels of Relatedness for Categories . . . . 84 
INTRODUCTION

In recent years psychologists have become concerned with the nature of semantic memory, which deals with word meanings and their structural representations. As aptly described by Tulving (1972), semantic memory is

"the memory necessary for the use of language. More explicitly, it is a mental thesaurus, organized knowledge a person possesses about words and verbal symbols, their meaning and referents, about relations among them, and about rules, formulas, and algorithms for the manipulation of these symbols, concepts and relations" (p.386).

The term "semantic memory" was first introduced in psychology by Quiltian (1967) in his dissertation (cited by Tulving, 1972). It originated from the necessity of assuming semantically organized material for the memory bank of the computer in an attempt to simulate a language comprehender. Subsequently, hypotheses and models of how humans might retrieve the meaning of words and facts from "semantic memory" were proposed by psychologists of the information processing approach who viewed the individual as an active agent, processing available linguistic information and possessing a highly organized permanent memory (Landauer and Freedman, 1968; Collins and Quil1ian, 1969; Meyer, 1972; Smith, Shoben and Rips, 1974; Glass and Holyoak, 1974, 1975, etc.).

Although the information processing approach represents one class of attempts to delineate the intricacies of semantic memory, this has not been the only approach undertaken in the history of American 
psychology. Earlier approaches originated with psychologists involved in the investigation of words and their associations (Bousfield, 1953; Osgood, 1952; Deese, 1962, 1965; Cofer, 1963; and Jenkins, 1964). The major research focus of this tradition has been to describe word meanings and, subsequently, to represent the organization of such meanings through constructing a network of associated words. Another approach has been a purely empirical one that attempts to describe the organization of the mental dictionary (Brown and Mciveil, 1966; Miller, 1969, 1972; and Fillenbaum and Rapoport, 1971). A variety of tasks, like tip-of-the-tongue phenomenon and semantic sorting techniques were employed to describe some of the regularities of storage and the semantic variables that categorize words as similar or dissimilar.

These research traditions share in common a view of memory as an organized body of knowledge. Semantic memory must represent an organized structure. Facts are connected to other facts in a non-random way. One word is connected to other words related to it in meaning. Accessibility and reconstruction of linguistic information is achieved without much effort, though not without error, as can be observed in human communication. Meaning of words and their relations or referents must be organized in a structure that makes their accessibility as well as their reconstruction highly possible.

A critical problem, according to the present author, that needs to be answered before any formal attempt to describe semantic memory can be undertaken is that of the representation of meaning in memory. Knowledge of how meanings of words are represented in memory will facilitate the difficult task of describing the structure of memory and 
the processes of retrieval from such a structure.

The various semantic memory models proposed in recent years are divided into network and set-theoretic models due to their different representations of word meanings. (For a detailed presentation of these models, see Appendix I.) In set-theoretic models (i.e. Meyer, 1970, 1973; Schaeffer and Wallace, 1970; Kintsch, 1972, 1974; Smith, Shoben and Rips, 1974) concepts are represented by a set of elements which might be exemplars, attributes, subsets or supersets of the concept. Semantic relations are defined in terms of operations, such as set inclusion and set exclusion. For example, the statement "a car is a vehicle" is verified by determining that the set of features defining "car" contains the set of features defining "vehicle". Furthermore, natural language concepts are assumed to have fuzzy edges whose boundaries are not sharply defined. Thus, the relation of category membership is assumed as a matter of degree. Instances show various degrees of relatedness to the category and some instances are better members of the category than others.

Network models (i.e. Collins and Quillian, 1969; Landauer and Freedman, 1968; Conrad, 1972; Glass and Holyoak, 1974, 1974/1975) assume that words, or their counterparts, exist as independent units in semantic memory connected by a network of labeled relations. Class inclusions and exclusions are specified by the graphic presentation of networks. For example, if an instance is connected to a superordinate, it signifies that the instance is contained in the category that the superordinate belongs to. However, if there is no connection between an instance and a superordinate, it is assumed that the instance is not 
contained in the category of that particular superordinate. In contrast to the set-theoretic models, semantic relations are all-or-none. A semantic component either dominates another or it does not, and a component either contradicts another or it does not. This type of representation leads to absolute rather than continuous notions of truth and falsity of semantic relations. For example, a person might be uncertain as to the truth value of "a bat is a bird", due to his ignorance or the sentence's ambiguity; but, nevertheless, an absolute dichotomy between the notions of truth and falsity is assumed.

In addition to the question of meaning representation, other empirical questions about the processes and variables affecting retrieval have been raised. Specifically, the question has been raised as to whether retrieval processes are simultaneous or parallel (Landauer and Freedman, 1968; Homa, 1973; and Meyer, 1973). There is also the question of what the nature of these processes is. More specifically investigators have asked whether retrieval of an inference that is repeated twice facilitates this retrieval step (Collins and Quillian, 1970a), what are the directional effects of inclusion relations (Loftus and Bolton, 1974), whether subjects use differential processing for various categories (Freedman and Loftus, 1974), whether the search for adjectives involves a different process than search for nouns (Grober and Loftus, 1974; and Loftus and Cole, 1974) and, lastly, what are the processes underlying rejection of false but meaningful sentences. The effect of a number of structural variables related to semantic memory findings have been investigated. Attention has been directed towards the frequency with which a particular 
instance is produced when a category name is presented (known as instance dominance) and the frequency of a category name when an instance is presented (known as category dominance) (Wilkins, 1971; Loftus and Freedman, 1972; Loftus and Suppes, 1972; Loftus, 1973; Shoben and Rips, 1973; Anderson and Reder, 1974; Glass and Holyoak, 1974; Glass, Holyoak, and O'De11, 1974; Sanford and Seymour, 1974). Another variable whose effect has been studied is relatedness, the degree to which an instance is related to its category, measured by asking subjects to rate how related or typical an instance is to its category (Rips, Shoben and Smith, 1973; Smith, Shoben and Rips, 1974; and Rosch, 1975). And lastly, category size (i.e. the number of instances that a category contains) has been scrutinized as a storage variable that could affect retrieval (Landauer and Freedman, 1968; Collins and Quillian, 1970b; Meyer, 1960; Wilkins, 1971; Freedman and Loftus, 1971, 1974; Landauer and Meyer, 1972; Homa, 1973; and Smith, Shoben and Rips, 1974).

A variety of tasks have been used to test the validity of the semantic memory models and the effects of the variables under question. Subjects have been required to identify whether common English words do or do not belong to well-known verbal categories (Landauer and Freedman, 1968; Collins and Quillian, 1970b; Wilkins, 1972; Smith and Haviland, Buckley and Sack, 1972; Loftus, 1973; Rips, Shoben and Smith, 1973; Sanford and Seymour, 1974; Anderson and Reder, 1974), to ascertain the truth or falsity of a sentence such as "a collie is a dog", or "al1/ some/few/many/no collies are dogs" (Collins and Quillian, 1969; Conrad, 1972; Rips, Shoben and Smith, 1973; Smith, Shoben and Rips, 1974; 
Holyoak and Glass, 1974, 1975), to judge or to designate whether two words have the same or different meaning (Schaeffer and Wallace, 1970), to designate whether a target word belongs to either of two designated categories (Meyer, 1973) and to produce a word that satisfies various specified restrictions, such as a word that names a member of the category "fruits" or a word that names a member of the category "animals" and also begins with the letter "Z" (Loftus, Freedman and Loftus, 1970; Freedman and Loftus, 1971, 1974; Loftus and Freedman, 1972; Glass and Holyoak, 1974; Grober and Loftus, 1974; Loftus and Bolton, 1974; Loftus and Cole, 1974; and Holyoak and Glass, 1975). Some of these tasks require subjects to identify whether or not two words have same or different meanings and whether or not a given instance is a member of a particular class or category, while other tasks require the subjects to produce a word.

The selection of such tasks reveals the underlying assumption made by these experimenters that categorization of some sort is the basic structure of word memory. For network models, the categorization is seen as an all-or-none phenomenon, while for the set-theoretic models degrees of categorization are hypothesized. It is imperative, however, to offer evidence regarding the soundness of these basic assumptions. The phenomena of chunking, categorical and subjective organization observed is free recall experiments of word lists, though widely used, are not adequate evidence to support such assumptions. The need for evidence becomes especially clear when one considers the issue of retrieval versus storage. With classification experiments the question arises whether the observed reaction time is due to getting information out of the storage based on categories or due to 
time needed to compute or infer based on bits and pieces of information stored in several different places. In other words, the observed reaction time could be due to output variables that organize the material before they are spewed out or to the structurat variables of the specific memory. If the observed times are not due to structural but output variables, then, it is a great fallacy to base models of storage on such experiments.

Sentence verification and classification experiments contain further problems. Such tasks involve not only retrieval from semantic memory and understanding, but also interpretation of the idea expressed through the sentence and experimenter's requirements in the tasks. For example, one may understand that "a chicken is a bird" without accepting that the sentence is correct. To render it as correct, one might like to see it read "broadly speaking, a chicken is a bird". Similarly, one understands that "a sparrow is a singing bird" but may not agree with the sentence. One may believe that a sparrow chirps and does not sing. Thus, interpretation and verification of such sentences depend on the definitions given by the subject to the critical words "bird" and "sing". Also in sentence like "some mountains are Alps" there is an implication in "some" that would render this sentence false for many people (i.e. if only some are, then some are not). Therefore, the subject might believe that in some instances, the experimenter wants him/her to use the broader definition of words and of others their narrower definitions. It is clear then that such tasks combine understanding and retrieval from memory with interpretation of the expressed idea leading to confounded conclusions on the structure 
of memory.

Dissatisfaction with the verification and classification tasks has been nicely expressed by Johnson-Laird (1974, p.142):

"The [semantic memory] experiments may even pass by the fundamental problem [of human semantic processing] since it is so rare in everyday life to have to ascertain the truth of a sentence such as "a dog is an animal". Of course, one can hardly understand such a sentence without verifying it. But there is a considerable distinction between these two transactions for ordinary contingent sentences like "a dog is expensive to keep". In understanding this sentence, an individual presumably retrieves some information from the lexical entry for "dog", but what? It seems that for the most important semantic retrieval problem, we have no clear idea of what is retrieved, let alone how it is retrieved."

The present research is directed to exactly this question, what is retrieved from human memory for the meanings of words.

This question of what is retrieved from semantic memory has been given attention by recently proposed models (Smith, Shoben and Rips, 1974; and Glass and Holyoak, 1974/1975). The two models whose views about meaning representations are reviewed in this paper, are the ones that include a formal and somewhat detailed treatment of word meanings and their representations in memory. Smith et a1. (1974) have proposed a feature-comparison model that stems from the set-theoretic tradition. In brief, this model assumes that a semantic category can be represented in memory as a set of features. These features vary in the extent to which they define that category. In other words, there is a continuum along which some features will be more essential in defining the category (called defining features) and others will be relatively unimportant or non-essential (called characteristic features). The features of each item are presumed to be ordered by degree of defining- 
ness with features that are more defining being at the top and features that are least defining being at the bottom. In addition, there is presumed to be an arbitrary boundary creating the distinction between defining and characteristic features. The boundary depends on the implicit weights attached to each feature. The number of defining features contained in an item's meaning are assumed to decrease as the item becomes increasingly abstract. This assumption seems unavoidable when one views meaning of words in terms of defining features, since abstract words should have defining features that encompass a number of instances. In retrieval from memory the entire set of features is accessed and for accurate judgments of class inclusions or exclusions defining features are given more attention.

A network model has been proposed by Glass and Holyoak (1974/ 1975) that has incorporated the marker structure as described by Katz (1972). Words are associated with a single marker in the attribute structure, referred to as the "defining" marker. For example, "avian" is the defining marker for "bird". Markers are best thought of as semantic properties of that word and the marker "canary" stands for an abstract concept equivalent to "possessing the essential properties of a canary" (Glass and Holyoak, p.14). However, markers are interrelated in such a way that any marker stands for, or dominates, a set of further markers associated with it. Therefore, class membership for an instance and its category is represented by the "defining" marker of the category that also dominates the defining marker of the instance, while class exclusion is represented by the lack of common marker between the word and the category in question. In fact, when 
such a common marker does not exist ambivalence arises. Contradictions are structurally represented in the system through association between markers. The structural representations of the contradictions and how some associations among markers are labeled as contradictory is not clear. However, the essential point that this model makes is that intersections are of two types - contradictory and non-contradictory.

Clearly, the marker model proposed by Glass and Holyoak (1974/ 1975) comes in opposition to the representation of meaning as outlined by the feature-comparison mode1. Markers are only defining properties and there are no significant, semantic properties of a word that can be considered "characteristic", as Smith et a1. (1974) proposed. Characteristic features are, according to Glass and Holyoak (1974/1975), "defining" markers of other words that are dominated by the "defining" markers of the word in question. It is pertinent, therefore, to review the evidence of the characteristic features assumption.

The major linguistic evidence presented by Smith et a1. (1974) is Lakoff's analysis of hedges (1972). Hedges are a class of modifiers whose major function seems to be that of qualifying predicates - some of such hedges being "technically speaking", "loosely speaking", "a true" etc. (i.e. a robin is a true bird). Lakoff hypothesized the existence of characteristic features to explain why sentences with different hedges show different degrees of acceptability as meaningful sentences. In other words, if the hedge "a true" in the sentence "a robin is a true bird" is replaced by "loosely speaking", the new sentence is not acceptable as a meaningfur one.

Further linguistic evidence is drawn from Sapir (1944) and Bierwisch (1971) in their analysis of relative adjectives. Basically 
they propose that when a relative adjective modifies a particular subordinate term the sentence is understood in relation to the characteristic feature, implied by the modifier, contained in the meaning of the superordinate term. To illustrate this point, Smith et al. uses this example:

Consider Sentence A:

(A) That bird is big.

In order to evaluate the truth of this sentence, one needs to compare the size of the designated bird with some size norm. Following Bierwisch's argument, this norm often might be the average size of the direct superordinate class; that is, the average size of a bird. Hence, sentences $B$ and $C$ are paraphases of $A$ :

(B) That bird is big for a bird.

(C) That bird's size is bigger than the average size of a bird.

Thus, a sentence like A contains an implicit comparison to a particular dimension value that characterizes the class to which the subject noun belongs, and this comparison is made explicit in C. Phrased somewhat differently, the superordinate class contains characteristic features (e.g. a particular average size) and constructions like $A$ are understood in relation to these characteristic values (Smith et a1., 1974, p.218).

Experimental evidence for the characteristic features assumptions is presented by Rosch (1973) and Rips et al. (1973). Subjects were presented with a set of instance-category pairs (e.g. fruit apple, fruit - fig, etc.) and were asked to rate how typical or how good an example of a category various instances were. It was found that agreement among subjects was high, especially in ranking the "best examples" of categories and that these results were highly replicable (Rosch, 1974). Moreover, the typicality ratings were reported as being 
in excellent congruence with the results of Lakoff's (1972) hedge analysis, in that instances rated as very typical (e.g. "robin" and "sparrow" for the category "bird") are those that are judged as meaningful when modified in a sentence by the hedge "a true", while instances judges to be atypical (e.g. "chicken" and "duck") are acceptable with the hedge "technically speaking". In light of these results, Smith et al. (1974) concluded that typicality ratings reflect the extent to which the characteristic features of a superordinate are similar to the features of an instance. The defining features of a superordinate cannot exercise much influence on such ratings because all instances contain these features.

Further results that support the characteristic feature assumption are presented by Rosch (1974). She had a group of subjects generate sentences for superordinate terms. An example of a sentence generated for the superordinate "bird" is "the tree has about twenty birds perched in it". Rosch then substituted an instance for the superordinate term in the constructed sentence, where this instanced varied in how typical it was of the superordinate (e.g. "robin" or "chicken" might be substituted for "bird" in the above example). Then another group rated the altered sentences for acceptability as meaningful sentences. The major finding was that rated acceptability decreased as typicality decreased. The explanation offered by Rosch was in terms of characteristic features of the superordinate. The sentence generated by the first group had a congruence between the characteristic features of the superordinate (e.g. "bird") and the predication in the sentence (e.g. perch in trees). It was inferred that this congruence 
was violated when atypical instance was substituted, since an atypical instance does not share the specific characteristic features implied by the predicate of the sentence.

It should be noted that the evidence offered thus far for the characteristic feature assumption is indirect. The assumption of characteristic features has been invoked to explain various results. As one might surmise from the indirectness of the evidence, different interpretations of such results have been offered and, consequently, the distinction between characteristic and defining features remains in some doubt.

One different explanation has been offered by Glass and Holyoak (1974/1975) who claimed that Lakoff's (1972) analysis of hedges can be reinterpreted in light of dual definitions for common English words. One is a popular definition and another is a technical definition, first agreed upon for some specific purpose by a select group, and eventually imposed on the general public. The definition of a bird as a small flying animal with wings is an example of a popular definition, while the biological definition of a bird is an example of a technical definition. Thus, the hedge "a true" signifies an instance of the category that fulfills the requirements of both the technical and popular definitions (as "robin" is for "bird"). The hedge "technically speaking" is used when an instance fulfills only the technical definition (e.g. "chicken" for "bird"), and the hedge "loosely speaking" is used when an instance satisfies only the popular definition (e.g. "bat" for "bird").

In view of this alternative explanation the present experiments 
were designed to investigate more directly the characteristic-features assumption. They were addressed to the question of whether both defining and characteristic features possess the qualities attributed to them. More specifically, the degree to which relatedness norms for instances reflect differences in the overlap of both types of features for the instance and the category name was investigated. Relatedness norms reflect the degree of relationship of the instance to its category. Such norms were obtained by Rosch (1975) who instructed subjects to rate, on a 7-point scale, the extent to which each instance (e.g. "robin" or "vulture") represented their idea or image of the meaning of the category term (e.g. "bird"). Lakoff (1972) in his analysis of hedges suggests that relatedness norms reflect the extent to which some instances share with the category both defining and characteristic features (a highly related instance), while others share only defining features (a moderately related instance) or characteristic features (a low related instance).

In Experiment 1, frequencies of features were collected for several semantic categories and their instances selected to represent different levels of relatedness. In Experiment 2, the subjects of the first experiment separated the features that they had given into defining and characteristic categories. In Experiment 3, a new group of subjects was presented with many of the features generated in Experiment 1 and was asked to separate them into defining and characteristic categories.

The central hypotheses of the study were the following:

(1) Defining features of a word are more central to word 
meaning than characteristic features;

(2) The number of defining features in an item's meaning increases or decreases with the degree of abstractness;

(3) Relatedness norms reflect the structure of semantic categories that can be analyzed to specific kind(s) of features that a category shares with its instances. As the specific kind(s) of features varies, relatedness measure of the instance to the category also varies.

The following specific predictions, as derived respectively from the three hypotheses, were tested:

$\left(A_{1}\right)$ Subjects would give more defining than characteristic features for a word when asked to provide properties or features for that word because defining features carry more weight in defining a word.

$\left(A_{2}\right)$ There would be more defining features given as the first feature of a word than characteristic features.

(B) The number of defining features provided for an instance would be greater than the number of defining features given for its superordinate.

(C) The correlation of defining and characteristic features of an instance with the defining and characteristic features of its category would vary with the degree of relatedness of the instance to the category. More specifically (based on Lakoff (1972), $\left(C_{7}\right)$ : highly related instances of a category would show a correlation between the defining and characteristic features of the instance and those of the defining and characteristic features of their category: $\left(C_{2}\right)$ : moderately related instances would show a correlation only between their 
defining features and those of their category; and $\left(\mathrm{C}_{3}\right)$ : low related instances would show a correlation between their characteristic features and those of their category name. 


\section{EXPERIMENT 1}

Before attempting to examine the internal structure of mental representations of semantic categories, it was necessary to obtain frequencies of features for a number of categories and their instances. Such frequencies must be obtained empirically because features that appear in dictionary definitions do not necessarily reflect the operating knowledge of word meanings by any specified population, since they are intended for special groups, i.e. scientific dictionaries, children's dictionaries, etc. It would be incautious to assume that most users of a word know its exact dictionary definition.

METHOD

\section{Subjects}

One hundred and five volunteer students from several introductory psychology classes at the University of Rhode Island participated in this study. Onty native speakers of English were included as found by asking the subjects to write down their country of birth and languages spoken frequently. This criterion did not eliminate any of the participating subjects.

Sex of the subjects was not treated as a variable following the research in this area (Landauer and Freedman, 1968; Collins and Quillian, 1969; Meyer, 1971; Smith et a1., 1974 etc.) showing that asking subjects to define common English words is not influenced by the sex of the subjects. Similarly, age of the subjects was not treated as a 
variable, since all subjects were of typical college age.

A11 subjects received extra credit for their participation in this task. Although the subjects were rewarded volunteers the representation of the sample was not restricted, since of the subjects asked to participate $100 \%$ of them took part in this experiment. Such reward was not expected to influence the subjects' verbal responses in any systematic way as there were no right or wrong answers. Stimuli

The category names for which features were to be gathered were chosen from Rosch's (1975) categories on which relatedness norms appear for a number of their instances (see Rosch, 1975, for reliability measures). Selection proceeded in the following manner: Categories were excluded if (a) their name was not a single word; (b) instances of a category could possibly overlap with instances from another category (e.g. "toy" and "weapon"); and (c) instances of a category was likely to be more familiar to one sex of subjects than another. The selected categories were: vegetable, fruit, bird, vehicle and furniture.

For each of these five categories four instances were selected from Rosch's norms to represent different levels of relatedness. However, instances across categories were utilized to represent same levels of relatedness (see Table 1).

Sixteen random orders of the 25 stimulus words were randomly selected with the restriction that two instances of the same category would be separated by a minimum of two non-instances. In this case, category names were treated as instances. 


\section{Procedure}

Subjects were tested in groups in their classes. They were given a 25-page booklet that had one stimulus word per page. They were also provided with a written copy of the instructions that read:

This study has to do with the question of what properties or features one has in mind when seeing a word. Let's take the word "robin" as an example. If you are asked to give the properties or features that you think of when you see the word "robin", you might say: "a robin is two-legged, has wings, is brown and red, perches in trees, is untamed, etc.". Similarly, if you are asked to give the features or properties that come in mind with "apple" you could say: "an apple is round, is yellow, is red, is green, has hard skin, shiny skin, soft inside, etc.".

When you turn this page, you will see a number of wellknown words, one word per page. Under each word are a number of spaces marked off. Think of the features or properties that come in mind when seeing each word and write them down in the provided spaces. Write one property or feature in each space. There is no limit of how many features you can give for a particular word, but do give all features that you can think of. There is no time limit for this task, but, please, work efficiently and quickly.

$\underline{\text { Results }}$

A11 responses made by each subject for categories and instances were tallied. The basic data, presented in Table 1 for each of the 25 stimuli, contain responses given with a total frequency of 10 or more. Category names appear first in the tables, followed by their instances arranged in a decreasing order of relatedness. The features have been ordered so that most frequent features appear first and least frequent 1ast. The two measures recorded are absolute frequencies in the first column and percentages in the second. 
FROM PRODIUTION MEMORY

\section{CATEGORY 1 \\ VEG PTABLP}

Frequencies percentages

1. is nutritious (healthy)

2. is green

3. is eतible

4. grows in gardens

5. is many varieties

6. eaten fresh

7. has vitamins

8. is yeliow

9. is a plant

10. is various color:

11. eaten at meals

12. is red.

13. can he cooked

14. is hara

15. grow: from soil

16. is a food

17. Grows from soil. (ground)

18. has many shapes

19. tastes gooi

20. is soft

21. can be cultivated
47

34

29

26

26

25

2.2

20

19

17

16

15

14

13

13

13

13

12

12

10
44.76

37.14

32.38

27.62

24.76

24.76

23.81

20.95

19.05

18.10

16. 19

15. 24

14.29

13.33

12.38

12.383

12. 38

12.38

11.43

11.43

9.52 
SPINACH

Frequencies Percentages

1. is greon

2. is a vegetable

3. has ]eaves

4. is nutritious (healthy)

5. can be cooked

6. has minerals (iron)

7. tastes bar

8. j.s inushy

9. has vitamins

10. tastes good

11. grows from soil

12. faten at meals

13. childien do not likə it.

14. has yceen leaves

15. grows in garảens

16. eaten raw
99

78

57

33

30

20

18

16

14

14

1.3

13

13

12

10

10
94.21

74.29

54.29

31.43

28.57

19.05

17. 14

15.24

13.33

13.33

12. 38

12.38

12.38

11.43

9.52

9.52 
TOM TO

Freguencies

102

60

57

50

39

36

35

30

29

26

22

21

17

16

14

10

10

10

10
Percentages

97.14

57.14

5.29

47.62

37.14

34.29

33.33

28.57

27.62

24.76

20.95

20.00

16.19

15.24

13.3 .3

9.52

9.52

9.52

9.52 


\section{PARSLEY}

Frequencies

1. is grcen

2. used for decoratiny foor

3. is a spice

4. used for seasoning

5. is a vegetable

8. can be dried up (flakes)

7. is $\sin 11$

10. has little or no taste

11. has stem

12. is edible

13. eaten raw
88

45

27

25

2.1

17

16

14

1.3

13

10
Percentages

83.81

42.96

25.71

23.81

20.00

16.19

15.24

13.3 .3

12. 38

12.38

12.38 
Frequencies Percentages

1. is yellow

69

2. is a flower

65

3. is a weed

56

4. has stem

5. has yellow flowers

6. grows in lawns

7. has green stem

8. qrows in grass

9. grows in the $511 \mathrm{mmor}$

10. blown away (for good luck)

11. has white fluff

$$
\text { (attached to seeds) }
$$

12. grows in the spring

13. has fuzzy seeds

14. has yellow petals

15. mầe into wine

16. has green leaves

17. removed from lawns (not liked)

18. is short

17. is peetty

20. is a food

21. is yreen

22. is white

23. is eriblo
20

43

34

33

25

2.4

2.3

20

16

16

15

15

14

14

13

13

12

11

10
64.75

61.90

53.33

40.95

32.38

31.43

23.81

22.86

19.05

19.05

18.10

15.24

15.24

14.29

34.29

13.33

13. 3.3

12.38

12.33

11.43

10.48

$9.5 ?$
21.90 


\section{CATBGORY 2 \\ PRUIT}

\section{Frequencies Percentages}

1. is juicy

2. grows on trees

3. is nutritious (healthy)

4. is swret

5. has many colors

6. tastes good

7. has seedis

8. is clible

9. many varieties

10. has vitamins

11. has skin

12. jrows on bushes

13. is soft (inside)

14. is sour

15. is various shapes

16. makes salads

17. is red

18. makes desserts

19. is various sizes
46

44

43

30

24

24

2.4

22

22.

16

15

14

1.3

12

11

10

10

10

10
4.3 .81

41.90

40.95

28.57

22.86

22.86

22.86

20.95

20.95

15. 24

14.29

13. 3.3

12. 38

11.43

10.58

9.52

9.52

9.52

9.52 
PI.IIM

Frequencies percentages

1. is purple

2. has a pit (seed)

3. is a Eruit.

4. is juicy

5. has skin

6. is soft inside

7. is small

8. is round (almost)

7. is sweet

10. grows on trees

11. is sour

12. is rea

13. tastes qood

14. grows in summer

15. can he cooked

16. is edihle

17. has shinny skin
67

66

87.62

6.3 .81

62.86

59

49

41

36

38

33

30

21

19

19

17

11

10

10
56.19

46.67

39.05

34.29

36.19

31.43

28.57

20.00

18.10

18.10

16. 19

10.48

9.52

9.52 
WATPRMION

Frequencias Percentages

1. has black and white seeds

92

87.62

2. is large (hig)

64

60.95

3. is juicy

63

60.00

4. grows in sumer

49

46.67

5. is red insidde

47

44.76

6. is a fruit

44

41.90

7. is green

41

39.05

8. is rinkish-red

.39

37.14

3. has green skin

37.14

10. has sind

29.52

11. is sweet.

27

25.71

12. is oval

26

24.76

13. contains water

2.5

23.81

14. tastes good

2.2

20.95

15. qrows in vines

21

20.00

16. is heavy

18

17.14

17. has white rind

15.24

19. stops thisst

19. is soft insido

12.38

20. has yreen rind

12.38

21. is finle

22. is rCd

10.48

23. is sticky

11

10.48 
COOSFREERY

Frequencies

26

22

20

17

17

16

10
Percentages

30.48

24.76

20.95

20.95

16.19

16.19

7. is round (almost)

15.24

8. is edible
9.52 
AVOCADO

Frequencies Percentages

1. is green

82

78.10

2. has a pit

R2.

78.10

3. js a fruit.

38

36.19

4. is a vegetable

24

22.86

5. is pear-shaped

22

20.95

6. has skin

20

19.05

7. is soft inside

$18 \cdot 10$

8. is table

18

17.14

9. has hacd skin

18

17.14

10. grows on trees

15

14.29

11. grows plants from pit

14

13.33

12. is a plant

12

11.43

13. tastes bad

11

10.48

14. can be eaten raw

11

10.48 


\section{CATGOSY 3}

BIRD

Frequencies

1. flins

2. has (two) wings

3. has beak (bill)

4. has (two) legs

5. has feathers

6. 1ays eggs

7. chirps

3. is small (1ittle)

9. huilds nests

10. eats worms

11. is multicolored

12. sings

13. perches in treos (hranches)

14. lives in nests

15. is an animal

16. has (two) feet

17. many species (types)

13. Migrates (flies south in winter)

13. is domestic (tamed)

20. is wild (untamed)

2.1. eats seeds

2.2. lives in trees

2.3. has (two) eyes
91

77

48

48

Percentages

86.67

73.33

45.71

45.71

42.86

28.57

28.57

24.76

23.81

45.71

2.2. 86

21.90

20.00

16.19

16.19

15.24

15.24

14.29

14.29

12.38

10.48

11

10.48

10

9.52 
page 32

SPARROW

Frequencies

Percentages

1. is a hird

81

77.14

2. is $\sin 11$ (1it+10)

68

64.76

3. has (t.wo) wings

58

55.24

4. EIi6s

48

45.71

5. has (two) logs

40

38.10

6. is hrown

36

34.29

7. has heak (bill)

22

20.95

8. sings

18

17.14

9. chirps

17

16.19

10. eats worns

17

16.19

11. has feathers

16

15.24

12. Iives in nests

14

13.33

13. moves fast

10

9.52

14. eats seeds

10

9.52

15. Lives in trees

10

9.52 
Frequencies Percentages

1. is a bird

r. has (two) logs

9. is a houso pet (domesticatad)

10. is multi-colored

11. flies

12. is green

13. has feathers

14. has large beak

15. lives in tropical areas

16. is large (big)

17. can be traincd

19. has (two) eyes
76

67

41

39

38

37

32.

29

72.38

63.81

39.05

37.14

36.19

35.24

30.48

27.62

24.76

20.95

19.05

19.05

16.19

16.19

15. 24

14. 29

13

12.38

11 
page 34

VULTURE

Frequencies

1. is a bird

2. preys on dead (or almost dead)

flesh

3. is large (big)

4. is black

5. has (two) wings

6. has heak (bi11)

7. fliss

8. is ugly

9. circles around proy (victim)

10. Iives in desert.

11. has large beak

12. has (two) legs

13. has feathers

14. has claws

15. is nreditory (bird of prey)

16. is bad (evil, mean)

17. has (two) eyes

18. symbol of death

19. is carnivorous
46

52

46

38

36

30

30

25

24

21

19

17

15

14

14

14

12

11

11
Percentages

43. 81

49.52

43.81

36.19

34.29

28.57

28.57

23.81

22.86

20.00

18.10

16.19

14.29

13.33

13.33

13.33

11.43

10.48

10.48 
Frequencies

89

2. wadales (walks funny)

3. lives in cold weather

$$
\text { (winter, snow) }
$$

4. is a hird

5. resembles tuxedo

6. has beak (bill)

7. swims

8. is an animal

9. cannot fly

10. Iives in Antartica

11. Las (two) legs

12. lives in North pole

13. catch fish

14. has wobbed feet

15. has (two) wings

16. is small (li.ttle)

17. Eound in zoo

18. Found in water
Percentages

$$
84.76
$$

56.19

$$
50.48
$$

44.76

38.10

27.62

24.76

$18 \cdot 10$

$18 \cdot 10$

16.19

14. 29

13.33

13.33

12.38

12.38

12.38

10.48

9.52 
CATERORY 4

FU VITURF
Percentages

1. made of wool

5. has $(1-4)$ legs

6. is supportive (put things on)

7. made of (covered with) cloth

9. a variety of sizes

9. made of plastic

14. is various colors

15. many types

16. made of cushions

17. Eound in most builäings
52

32

31

31

17

14

14

14

12

12

11

11

10

2. \\ 1

 \\ 7}

7

16

15

14

4

2

1

10
49.52

30.48

29.52

29.52

16.19

16. 19

15.24

14.29

13. 33

13. 33

13.33

11.43

11.43

10.48

10.48

9.52

9.52 


\section{Frequencies \\ Percentages}

10. is spluare

11. found in kitchen

12. is hard

13. has smooth (finish) top

14. a variety of sizes

15. made of plastic

16. is rectangular

17. used for sitting on (or at)

18. is a variety of shapes

19. made of glass

20. used for studying
86

63

57

48

38

30

26

25

25

2.0

$$
81.90
$$

60.00

54.29

45.71

36.19

28.57

24.76

2.3 .81

2.3 .81

19.05

18

17.14

16

15.24

15

14.29

14

13.33

14

13. 3.3

13

13. 68

12

11.43

11

10.48

10

9.52

10

9.52 
page 38

BOOKC.SP:

1. holds books

2. has shelves

3. made of wood

4. made of metal

5. found in 1 ibraries

6. holds trinkets (ornaments, candles)

7. is tall (high)

8. is a piece of furniture

9. found against (olose to) a wall

10. is hrown

11. holds objects

12. is large (wide)

13. found in stury room

14. used for homes
Frequencies

91

63

61

24

23

17

15

14

14

14

11

11

10

10
Percentages

86.67

60.00

$58 \cdot 10$

2.2 .86

21.90

$16 \cdot 19$

14.29

13.33

13.33

13. 3.3

10.48

10.48

9.52

9.52 
STOOL

Frequencies Percentages

1. has $(1-4)$ legs

79

60

57

32

4. used as a step (stand on)

5. is a type of seat

(small chair)

27.63

6. used as a'footrest

27

25.71

7. has a round top

23

21.90

8. j.s short (10w)

22

20.95

9. found in bars

22

20.95

10. is small (narrow)

20

19.05

11. has no back

19

18

18

17

17

12

75.24

57.14

54.29

30.48

12. is a piece of furniture

13. made of metal

14. is tall (high)

15. is hard

16. can he uncomfortable
18.10

17. 14

17. 14

16. 19

16.19

11.4 .3 


\section{WASPOBASK FT}

Frequencies percentages

1. holds waste (garhage)

82

78.10

2. made of motal

35

3.3 .33

3. is round

30.48

4. holds (oli) papers

28

16.67

5. made of plastic

2.5

23.81

6. is smelly (dirty)

20

19.05

7. is a container

20

19.05

3. is cylinärical

19.05

9. is various colors

11. emptied when full

12. is small (nasrow)

13. found near (under) desks

15. can be decorated 


\section{CAT FOOFY 5 \\ VFHICLE}

Freguencies Percentages

$\begin{array}{lcc}\text { 1. means of transpotation } & 70 & 66.67 \\ \text { 2. has wheels } & 51 & 48.57 \\ \text { 3. nefds a fare } & 22 & 20.95 \\ \text { 4. carries people (passengers) } & 16 & 15.24 \\ \text { 5. has engine (motor) } & 15 & 14.29 \\ \text { 6. is various colors } & 15 & 14.29 \\ \text { 7. is various sizes } & 15 & 14.29 \\ \text { 8. covers distance } & 15 & 14.29 \\ \text { 9. is driven } & 14 & 13.33 \\ \text { 10. is various shapes } & 13 & 12.38 \\ \text { 11. is dangerous (accidents) } & 12 & 11.43 \\ \text { 12. is fast } & 12 & 11.43 \\ \text { 13. made of metals } & 11 & 10.48 \\ \text { 14. has steering wheel } & 11 & 10.48 \\ \text { 15. is expensive } & 10 & 9.52\end{array}$


$C A R$

1. has wheels

2. meansi of transportation

3. takes yas

4. has engine (motoc)

5. is vacious colors

6. has steering wheel

7. is expensive

3. has (2 to 4) doors

9. is vacious sizes

10. has glass windows

11. is a vehicle

12. has seats

13. is dangerous (accidents)

14. is various makes

15. is fast

16. is various styles

17. has metalis frame

18. is driven

19. gnes on roads (highways, driveways)

20. has trunk

21. has 1 ights

22. nerds repairs

23. has ruhber tires

24. is fun for cruising
Frejuoncies

71

$5 \%$

42.

38

33

33

31

31

31

2.7

27

25

2.3

23

22

20

18

16

14

14

1.3

13

13

12
13.3 .3

percentages

67.62

43.52

40.00

36.19

31.43

31.43

29.52

29.52

29.52

28.42

25.71

23.81

21.90

21.90

20.95

19.05

17.14

15. 24

13.33

12. 38

12. .38

12. 38

11.43 
25. has horn

26. has a radio

27. has brakes

28. is a symbol of prestiqe (status) 10

29. is convenient.
12.

12

10

10
11.43

11.43

9.52

9.52

9.52 
TROLLEY

Frequencies Percentages

1. goes on tracks

2. means of transportation

3. found in San Francisco

4. carries people (passengers)

5. is old-fashioned

6. is a train like

7. has seats

8. run by elecrticity

9. attached to wire overhead

10. has wheels

11. found in cities

12. needs a conductor (driver)

13. has a bell

14. is noisy

15. has (glass) windows

16. is various makes

17. is open

18. is a vehicle

19. Found in Roston

20. goes on roads (highways,

drivenays)

21. goos uphilis
64

56

52

.34

32

27

2.3

22

20

20

18

17

15

14

13

12

11

11

11

10

10
19.05

53.33

49.52

32.38

30.48

25.71

21.90

20.95

19.05

19.05

17.14

16. 19

14.29

13.33

12.38

11.43

10.48

10.48

10.48

9.52

9.52 
SCOOTES

Frequenaies percentages

1. has wheels

?. chilaren's toy

3. has steering handle

4. may have engina (motor)

5. means of transportation

6. is red

7. is pushed with one foot

8. is a vehicle

9. made of metal

10. takes gas

11. is fast.

12. is driven

13. has seats
92

50

41

3.3

2.7

25

23

18

15

13

12

10

10
87.62

47.62

39.05

31.43

25.71

23.81

21.90

17.14

14.29

12.38

11.43

9.52

9.52 
SKATES

Frequencies Percentages

1. has sharp blades

4. has laces

5. used for hockey

6. used on ice

7. used for sport

8. is shoe like

9. is black

10. is fun

11. made of leather

12. means of transportation

13. can glide
77

42

34

34

29

28

41

21

20

18

14

13

12
73.33

40.00

32.38

32.38

27.62

26.67

20.00

20.00

19.05

17.14

13.33

12.38

11.43 


\section{EXPERIMENT 2}

As previously mentioned, Smith, Shoben and Rips' (1974) feature comparison model assumes that features which define a word, vary along a continuum of importance, from very-important-in-defining (defining features) to unimportant-in-defining (characteristic features). These features are presumed to be ordered according to degree of definingness with defining features at the top and characteristics at the bottom. In retrieval from memory, the entire set of features is retrieved including both defining and characteristic features. Since defining features are more essential for the meaning of a word and are also ordered first, one would expect that when asking subjects to produce features that define a word in a limited time span, defining features would be both greater in number than characteristic features and given earlier.

It is possible, however, that the total set of features that define some words contain more characteristic than defining features. Consequently, defining features could be less frequent in number than characteristic features when subjects are asked to define a word even if defining features are more central to word meaning. Even in such a case, however, one would expect the defining features to be given earlier if they are more basic of words' subjective definitions. Therefore, defining features would be more frequently given as first responses when subjects are asked to generate features that pertain to a specific word. 
An additional assumption made by feature models (H. Clark, 1970; Meyer, 1970; and Smith et al., 1976) is that the number of defining features contained in an item's meaning decreases as the item becomes increasingly abstract; for example, "robin" contains more defining features than "bird". Since frequencies of features were gathered in Experiment 1, this assumption vis-a-vis production memory could be tested, by asking these same subjects to separate the produced features into defining and characteristic categories.

METHOD

\section{Subjects}

Ninety-five students from Experiment 1 participated in this study. The ten subjects from the previous study that did not participate were absent. Subjects received course credit for their participation and of the students present in classes all of them took part in this experiment. For reasons described in detail in the Method section of Experiment 1, sex and age of the subjects were not treated as variables.

Stimuli

Stimuli were the same booklets used for Experiment 1, containing five categories and four instances per category. The order of presentation of the stimuli for each booklet was reversed from the last experiment.

Procedure

Subjects were tested in groups in their classrooms. Each subject was given the booklet that he/she had filled out in Experiment 1 . 
They were presented with a new set of instructions that they were asked to read. Specific instructions read:

This study has to do with separating the features or properties that you previously gave for a word into two categories: (1) properties that are essential for the meaning of the word, and (2) properties that are not absolutely necessary for the meaning of the word, but which further explain it. For example, remember the word "robin". The features we hypothesized that this word contains were: "a robin is two-legged, has wings, is brown and red, perches in trees and is untamed". Trying to separate the features of "robin" into necessary ones for the meaning of the word, one could decide that "is two-legged, has wings, is brown and red" are essential for the meaning of "robin". However, "perches in trees, is untamed" could be decided as not necessary to the meaning of the word, but further explaining it. Similarly, for "apple" we hypothesized that its features could be: "an apple is round, is yellow, is red, is green, has hard skin, shiny skin, soft inside". The necessary features for "apple" could be: "is round, has hard skin" but the not absolutely necessary features could be: "is red, is yellow, is green, shiny skin, soft inside".

Now, in front of you, you have the features that you gave for each word. Read the features or properties of each word carefully and try to separate them into essential for the meaning of the word and those not absolutely necessary for the meaning of the word. If you decide that a property is necessary put a $(+)$ sign next to it, but if you decide that a property or feature is not always necessary put a (-) sign next to it. There is no time limit for this task, but, please, work quickly and efficiently.

\section{RESULTS}

Responses given by each subject were tallied and the data are presented in Table 2. This table follows the order of presentation of features from Table 1 of Experiment 1. The two recorded measures are absolute frequencies and percentages in parentheses.

On the number of defining and characteristic features that each subject gave for five category names and four instances per category, a 
FREQIJENCLES AND PFRCENTAGES OF ESSENIIAL AND NON-ESSFNTIAL FEATURES FROM PRODUCTION MEMORY

\section{CATEGOPY 1 \\ VEGE'TABLE}

Essential

1. is nutritious (hea Ithy)

2. is green

3. is edible

4. grows in gardens

5. is many varieties

6. eaten fresh

7. has vitamins

8. is yellow

9. is a plant

10. is various colors

11. eaten at meals

12. is red

13. can be cooked

14. is hard

15. qrows from soil

16. is a food

17. grows in soil (ground)

18. has many shapes

19. tastes good

20. is soft

21. can be cultivated

\begin{tabular}{|c|c|c|c|}
\hline 27 & $(28.42)$ & 15 & $(15.79)$ \\
\hline 20 & $(21.05)$ & 17 & $(17.89)$ \\
\hline 14 & $(14.74)$ & 15 & $(15.79)$ \\
\hline 16 & $(16.84)$ & 11 & $(11.58)$ \\
\hline 13 & $(13.68)$ & 10 & $(10.53)$ \\
\hline 2.1 & $(22.11)$ & 3 & $(3.16)$ \\
\hline 14 & $(14.74)$ & 10 & $(10.53)$ \\
\hline 11 & $(11.58)$ & 11 & 111.58 \\
\hline 4 & $(4.21)$ & 13 & $(13.68)$ \\
\hline 7 & $(7.37)$ & 11 & $(11.58)$ \\
\hline 16 & $(16.84)$ & 0 & $(0.00)$ \\
\hline 11 & $(11.58)$ & 5 & $(5.26)$ \\
\hline 12 & $(12.63)$ & 2 & $(2.11)$ \\
\hline 9 & $(9.47)$ & 4 & $(4.21)$ \\
\hline 5 & $(5.26)$ & 8 & $(8.42)$ \\
\hline 5 & $(5.26)$ & 8 & $(8.42)$ \\
\hline 4 & $(4.21)$ & 7 & $(7.37)$ \\
\hline 4 & $(4.21)$ & 9 & $(9.47)$ \\
\hline 11 & $(11.58)$ & 0 & $(0.00)$ \\
\hline 8 & $(8.42)$ & 3 & $(3.16)$ \\
\hline$r$ & $(7.37)$ & 3 & $(3.16)$ \\
\hline
\end{tabular}

Non-essential 
22. has Ieaves 
SETNACH

\section{Essential}

1. is green

2. is a vegetable

3. has leaves

4. is nutritious (healthy)

5. can he cooked

6. has minerals (iron)

7. tastes bat

8. is mushy

9. has vitamins

10. tastes good

11. grows from soil

12. eaten at meals

13. chiluren do not like it

14. has green leaves

15. grows in gacdens

16. eaten raw

\begin{tabular}{|c|c|c|c|}
\hline 7 & $(7.37)$ & 83 & $(87.37)$ \\
\hline 6 & $(6.32)$ & 65 & $(68.42)$ \\
\hline 6 & $(6.32)$ & 46 & $(48.42)$ \\
\hline 26 & $(27.37)$ & 4 & $(4.21)$ \\
\hline 26 & $(27.37)$ & 1 & $(1.05)$ \\
\hline 10 & $(10.5 .3)$ & 8 & $(8.42)$ \\
\hline 15 & $(15.79)$ & 1 & $(1.05)$ \\
\hline 7 & $(7.37)$ & $B$ & $(8.42)$ \\
\hline 7 & $(7.37)$ & 4 & $(4.21)$ \\
\hline 11 & $(11.58)$ & 1 & $(1.05)$ \\
\hline 10 & $(10.53)$ & 3 & $(3.16)$ \\
\hline 9 & $(9.47)$ & 2 & $(2.11)$ \\
\hline 9 & $(9.47)$ & 1 & $(1.05\rangle$ \\
\hline 1 & $(1.05)$ & 3 & $(3.16)$ \\
\hline 4 & $(4.21)$ & 2 & $(2.11)$ \\
\hline 7 & $(7.37)$ & 1 & $(1.05)$ \\
\hline
\end{tabular}


TOMATO

1. is red (when ripe)

2. is juicy

3. has seeds

4. eaten raw

5. is alnost round

6. grows in gardens

7. is a vegetable

8. is green (when not ripe)

9. is skin-covered

10. makes sauce

11. is a plant

12. is a Eruit

13. grows in vines

14. can be cooked

15. is shinny

16. tastes good

17. is edible

13. made into juice

17. is smooth
Fssential

$25(26.32)$

$26 \quad(27.37)$

$19(20.00)$

$41(43.16)$

$5 \quad(5.26)$

$17 \quad(17.89)$

$6 \quad(6.32)$

$16(16.84)$

$8 \quad(8.42)$

$19(20.00)$

$9 \quad(9.47)$

$3(3.16)$

$7 \quad(7.37)$

$10(10.53)$

$6 \quad(6.32)$

$10 \quad(10.53)$

$5 \quad(5.26)$

$8 \quad(8.42)$

$4 \quad(4.21)$
Non-essential

$66(64.47)$

$27(2.8 .42)$

$32(33.68)$

$2 \quad(2.11)$

$30(31.58)$

$14(14.74)$

$27 \quad(28.42)$

$10 \quad(10.53)$

$19(20.00)$

$3(3.16)$

$10(10.5 .3)$

$16(16.84)$

$9 \quad(9.47)$

$1(1.05)$

$8 \quad(8.42)$

$0 \quad(0.00)$

$3 \quad(3.16)$

2 (2.11)

$6 \quad(6.32)$ 
PARSLF,

1. is green

2. used for decorating food

3. is a spice

4. used for seasoning

5. is a vegetable

8. can be dried up (flakes)

9. is small

10. has little or no taste

11. has stem

12. is edible

13. eaten raw

\section{Fssential}

$10(10.53)$

$31(32.63)$

$6 \quad(6.32)$

$7 \quad(7.37)$

$2(2.11)$

$7 \quad(7.37)$

$7 \quad(7.37)$

$11(11.58)$

$1(1.05)$

$6 \quad(6.32)$

$9 \quad(9.47)$
Non-essential

$68 \quad(71.58)$

$10(10.53)$

$17(17.89)$

$13(13.68)$

$7 \quad(7.37)$

$6 \quad(6.32)$

$7 \quad(7.37)$

$2(2.11)$

$10 \quad(10.53)$

$5 \quad(5.26)$

$1(1.05)$ 


\section{Essential}

1. is yellow

2. is a flower

3. is a weed

4. has stem

5. has yellow flowers

6. grows in lawns

7. has green stem

8. grows in grass

9. grows in summer

10. blown away (for good luck)

11. has white fluff

$$
\text { (attached to seeds) }
$$

12. grows in spring

13. has fuzzy seeds

14. has yellow petal

15. made into wine

16. has green leaves

17. remover from lawns

$$
\text { (not liked) }
$$

18. is short

19. is pretty

20. is a food

21. is green

2.. is white

23. is edible

$\begin{array}{llll}13 & (13.68) & 50 & (52.63) \\ 9 & (9.47) & 51 & (53.68) \\ 13 & (13.68) & 39 & (41.05) \\ 10 & (10.53) & 29 & (30.53) \\ 4 & (4.21) & 27 & (28.42) \\ 21 & (22.11) & 11 & (11.58) \\ 3 & (3.16) & 18 & (18.95) \\ 11 & (11.58) & 8 & (3.42) \\ 16 & (16.84) & 3 & (3.16) \\ 12 & (12.63) & 6 & (6.32)\end{array}$

$8 \quad(8.42) \quad 11(91.58)$

$11(11.58) \quad 6 \quad(6.32)$

$10 \quad(10.53) \quad 3 \quad(3.16)$

$\begin{array}{llll}1 & (1.05) \quad 9 & (9.47)\end{array}$

$11(11.58) \quad 0 \quad(0.00)$

$\begin{array}{llll}3 & (3.16) \quad 9 & (9.47)\end{array}$

$13(13.68) \quad 1 \quad(1.05)$

$\begin{array}{cccc}6 & (6.32) & 7 & (7.37) \\ 8 & (8.42) & 3 & (3.16) \\ 10 & (10.53) & 1 & (1.05) \\ 2 & (2.11) & 9 & (9.47) \\ 2 & (2.11) & 8 & (8.42) \\ 7 & (7.37) & 1 & (1.05)\end{array}$


page 56

\section{Essential}

1. is juj.cy

2. grows on trees

3. is nutritious (healthy)

4. is sweet

5. has many colors

6. tastes good

7. has seeds

8. is edible

9. many varieties

10. has vitamins

11. has skin

12. grows on bushes

13. is soft (inside)

14. is sour

15. is various shapes

16. makes salads

17. is IEd

18. makes äesserts

19. is various sizes

\section{CATEGORY 2 \\ FPUIT \\ CATEGORY 2}

\begin{tabular}{|c|c|c|c|}
\hline 21 & $(22.11)$ & 20 & $(21.05)$ \\
\hline 19 & $(20.00)$ & 22 & $(23.16)$ \\
\hline 32 & $(33.68)$ & 1 & $(1.05)$ \\
\hline 16 & $(16.84)$ & 12 & $(12.63)$ \\
\hline 18 & $(18.95)$ & 5 & $(5.26)$ \\
\hline 19 & $(20.00)$ & 4 & $(4.21)$ \\
\hline 6 & $(6.32)$ & 16 & $(16.84)$ \\
\hline 6 & $(6.32)$ & 14 & $(14.74)$ \\
\hline 9 & $(9.47)$ & 11 & $(11.58)$ \\
\hline 9 & $(9.47)$ & 6 & $(6.32)$ \\
\hline 1 & $(1.05)$ & 12 & $(12.63)$ \\
\hline 6 & $(6.32)$ & 8 & $(8.42)$ \\
\hline 5 & $(5.26)$ & 7 & $(7.37)$ \\
\hline 7 & $(7.37)$ & 4 & $(4.21)$ \\
\hline 7 & $97.37)$ & 4 & $(4.21)$ \\
\hline 9 & $(1.05)$ & 1 & $(1.05)$ \\
\hline 7 & $97.37)$ & 3 & $(3.16)$ \\
\hline 10 & $(10.53)$ & 0 . & $(0.00)$ \\
\hline 6 & $(6.32)$ & 4 & $(4.21)$ \\
\hline
\end{tabular}

$20 \quad(21.05)$

Non-essential

\section{.}


PI, UM

1. is purple

2. hasia pit (a sefd)

3. is a fruit.

4. is juicy

5. has skin

6. is soft inside

7. is small

8. is round (almost)

7. is sweet

10. grows on trees

11. is sour

12. is red

13. tastes good

14. grows in summer

15. can he cooked

16. is edible

17. has shinny skin

$$
\text { Essential }
$$$$
23(24.21)
$$$$
2.7(28.42)
$$$$
5 \quad(5.26)
$$$$
30 \quad(31.58)
$$$$
13(13.68)
$$$$
18 \quad(18.95)
$$$$
10(10.53)
$$$$
2(2.11)
$$$$
20 \quad(21.05)
$$$$
17(17.89)
$$$$
16(16.84)
$$$$
6 \quad(5.32)
$$$$
12(12.63)
$$$$
12(12.63)
$$$$
7 \quad(7.37)
$$$$
1(1.05)
$$$$
5 \quad(5.26)
$$

Non-essential

$58 \quad(61.05)$

$31(32.63)$

$55 \quad(57.89)$

$20 \quad(21.05)$

$29(30.53)$

$16(16.84)$

$19(20.00)$

$30 \quad(31.58)$

$9 \quad(9.47)$

$8 \quad(8.42)$

$2(2.11)$

$8 \quad(8.42)$

$1 \quad(1.05)$

$2 \quad(2.11)$

$0 \quad(0.00)$

$6 \quad(5.32)$

4 (1t.21) 
WNTRMELON

Fssential

1. has black and white seeds

2. is lacqe (big)

3. is juicy

4. grows in summer

5. is red inside

6. is a fruit

7. is green

8. is pinkish-red

9. has green skin

10. has rind

11. is sweet

12. is oval

13. contains water

14. tastes good

15. grows in vines

16. is heavy

17. has white rind

18. stops thirst

19. is soft inside

20. has green rind

21. is edihle

2.2. is red

23. is sticky
$28(29.47)$

$23(24.21)$

$28 \quad(29.47)$

$35(36.84)$

$7 \quad(7.37)$

$4 \quad(4.21)$

$5 \quad(5.26)$

$11(11.58)$

$8 \quad(8.42)$

$8 \quad(8.42)$

$2.1 \quad(22.11)$

$4 \quad(4.21)$

$9 \quad(9.47)$

$17(17.89)$

$10(10.53)$

$11(11.58)$

$5 \quad(5.26)$

$11(11.58)$

$6 \quad(6.32)$

$3(3.16)$

$8 \quad(8.42)$

$2(2.11)$

$8 \quad(8.42)$
Non-essential

$56 \quad(58.95)$

$34(35.79)$

$28 \quad(29.47)$

$8 \quad(8.42)$

$34 \quad(35.79)$

$33(34.74)$

$30 \quad(31.58)$

$26(2.7 .37)$

$26 \quad(27.37)$

$19(20.00)$

$5 \quad(5.26)$

$21(22.11)$

$13(13.68)$

$2(2.11)$

$8 \quad(8.42)$

$5 \quad(5.26)$

$10 \quad(10.53)$

$1 \quad(1.05)$

$4(4.21)$

$10 \quad(10.53)$

$3 \quad(3.16)$

$7 \quad(7.37)$

$2 \quad(2.11)$ 
GOOSERERRY

\begin{tabular}{cccc} 
Fissential & \multicolumn{3}{c}{ Non-essential } \\
8 & $(8.42)$ & 20 & $(21.05)$ \\
6 & $(6.32)$ & 20 & $(21.05)$ \\
0 & $(0.00)$ & 19 & $(20.00)$ \\
18 & $(18.95)$ & 1 & $(1.05)$ \\
1 & $(1.05)$ & 14 & $(14.74)$ \\
9 & $(9.47)$ & 6 & $(6.32)$ \\
1 & $(1.05)$ & 12 & $(12.63)$ \\
4 & $(4.21)$ & 4 & $(4.21)$
\end{tabular}

1. qrows on bushes

2. is a kind of herry

3. is a Eruit

4. can be cooked

5. is small

6. is red

7. is cound (almost)

8. is fdible 
A VOCADO

1. is green

2. has a pit

3. is a Eruit

4. is a vegetable

5. is pear-shaped

6. has skin

7. is soft insine

8. is edible

9. has hard skin

10. grows on trees

11. grows plants from pit

12. is a plant

13. tastes bad

14. can be eaten raw

\section{Fssential}

$17(17.89)$

$16(16.84)$

$4 \quad(4.21)$

$5 \quad(5.26)$

$4 \quad(4.21)$

$2(2.11)$

$7 \quad(7.37)$

$7(7.37)$

$1(1.05)$

$10(10.53)$

$12(12.6 .3)$

$4 \quad(4.21)$

$10 \quad(10.53)$

$9 \quad(9.47)$
Non-essential

$59(62.11)$

$21(22.11)$

$30(31.58)$

$14(14.74)$

$15(15.79)$

$15 \quad(15.79)$

$10(10.53)$

$8 \quad(8.42)$

$14(14.74)$

2. (2.11)

$0 \quad(0.00)$

$7(7.37)$

$1 \quad(1.05)$

$0(0.00)$ 
CATBCORY 3

BI RD

Fssential

$33(34.74)$

$3(3.16)$

$11(11.58)$

3. has heak (bill)

4. has (two) legs

5. has feathers

6. Lays eggs

7. chirps

8. is small (little)

9. builds nests

10. eats worms

11. is multi-colored

12. sings

13. perches in trees (branches)

14. Iives in nests

15. is an animal

16. has (two) feet

17. many species (types)

18. migrates (Elies south

$$
\text { in winter) }
$$

19. is domestic (tamed)

20. is wild (untamed)

21. eat.s seeds

22. Iives in trees

23. has (two) eyes
$4 \quad(4.21)$

$2(2.11)$

$8 \quad(8.42)$

$24(25.26)$

$15(15.79)$

$18 \quad(18.35)$

$6 \quad(6.32)$

$13(13.68)$

$3 \quad(3.16)$

$18 \quad(18.95)$

$6 \quad(6.32)$

$9 \quad(9.47)$.

$3(3.16)$

$10 \quad(10.53)$

$11(11.58)$

$12(12.63)$

$11(11.58)$

$10(10.53)$

$4 \quad(4.21)$
$9(9.47)$
Non-essentia 1

$47(49.47)$

$64(67.37)$

$33(34.74)$

$39(41.05)$

$39(41.05)$

$18 \quad(18.95)$

$5 \quad(5.26)$

$7 \quad(7.37)$

$4 \quad(4.21)$

$18 \quad(18.95)$

$8 \quad(8.42)$

$18 \quad(18.95)$

$1 \quad(1.05)$

$10(10.53)$

$7 \quad(7.37)$

$11(11.58)$

$5 \quad(5.26)$
2 (2.11)

$1(1.05)$

$0 \quad(0.00)$

$1 \quad(1.05)$

$2(2.11)$

$6 \quad(6.32)$ 
SPARROW

1. is a hird

2. is small (little)

3. has (two) wings

4. flies

5. has (two) legs

6. is brown

7. has beak (bill)

8. sings

9. chirps

10. eat.s worms

11. has feathers

12. lives in nests

13. moves fast

14. eats seeds

15. lives in trees
Essential

2. (2.11)

$19(20.00)$

$6 \quad(6.32)$

$15(15.79)$

2 (2.11)

$10(10.53)$

$6 \quad(6.32)$

$10(10.53)$

$12(12.63)$

$13(13.48)$

$1(1.05)$

$10(10.53)$

$6 \quad(6.32)$

$7 \quad(7.37)$

$7 \quad(7.37)$
Non-essential

$72(75.79)$

$40 \quad(42 . .11)$

$46(48.42)$

$27 \quad(28.42)$

$34 \quad(35.79)$

$23(24.21)$

$14(14.74)$

$6 \quad(6.32)$

$4 \quad(4.21)$

$4 \quad(4.21)$

$14(14.74)$

$1 \quad(1.05)$

$2 \quad(2.11)$

$1 \quad(35.79)$

$0 \quad(0.00)$ 
PARROT

1. is a bicd

2. can talk

3. is colorful

4. has beak (bill)

5. Lives in cage

6. has (two) wings

7. can mimick (imitate)

8. has (two) legs

7. is a house pet (domesticated)

10. is multi-colored

11. flies

12. is green

13. has feathers

14. bas large beak

15. Lives in tropical areas

16. is large (big)

17. can be trained

18. has (two) eyes

$$
\text { Fisential }
$$$$
2(2.11)
$$$$
32(33.68)
$$$$
10(10.53)
$$$$
4 \quad(4.21)
$$$$
30(31.58)
$$$$
2(2.11)
$$$$
14(14.74)
$$$$
3(3.16)
$$$$
22(23.16)
$$$$
12(12.6 .3)
$$$$
6 \quad(6.32)
$$$$
9(9.47)
$$$$
1(1.05)
$$$$
1 \quad(1.05)
$$$$
4 \quad(4.21)
$$$$
10(10.53)
$$$$
8 \quad(8.42)
$$

$1 \quad(1.05)$
Non-essential

$68 \quad(71.58)$

$27 \quad(28.42)$

$23(24.21)$

$31(32.63)$

$2(2.11)$

$33(34.74)$

$15(15.79)$

$23(24.21)$

$2(2.11)$

$8 \quad(8.42)$

$12(12.63)$

$10 \quad(10.53)$

$14(14.74)$

$13(13.68)$

$8 \quad(8.42)$

$5 \quad(5.26)$

$2(2.11)$

$8 \quad(8.42)$ 
VIILTURF

1. is a bird

2. preys on dead for almost dead) flesh

3. is large (big)

4. is black

5. has (two) wings

6. has heak (bi11)

7. Elies

8. is ugly

9. circles around prey (victim)

10. Iives in desert.

11. has large beak

12. has (two) legs

13. has feathers

14. has claws

15. is preditory (bird of prey)

16. is bad (evil, mean)

17. has (two) eyes

18. symbol of death

19. is carnivorous

\section{Fissential}

$4 \quad(4.21)$

Non-essential

$72(75.79)$

$22(23.16)$

$23(24.21)$

$14(14.74)$

$26(27.37)$

$8 \quad(8.42)$

$28(29.47)$

$2(2.11)$

$32(33.68)$

$8 \quad(8.42)$

$20(21.05)$

$14(14.74)$

$13(13.68)$

17 (17.89)

$8 \quad(8.42)$

$11(11.58)$

$1(1.05)$

$10 \quad(10.53)$

$8 \quad(8.42)$

$7 \quad(7.37)$

$12(12.63)$

$0 \quad(0.00)$

$16(16.84)$

2 (2.11)

$12(12.63)$

$1 \quad(1.05)$

$11(11.58)$

$5 \quad(5.26)$

$8 \quad(8.42)$

$10(10.53)$

$3 \quad(3.16)$

$1 \quad(1.05)$

$10 \quad(10.53)$

$11(11.58)$

$0(0.00)$

$2(2.11)$

$6 \quad(6.32)$ 
[F.MIIIN

Essential

1. is black and white

2. wadrles (walks funny)

3. Lives in cold weather

$$
\text { (winteI, snow) }
$$

4. is a bicd

5. resembles tuxedo

6. has beak (bi11)

7. swims

8. is an animal

9. cannot fly

10. Iives in Antartica

11. has (two) leqs

12. Iives in North Pole

13. catch fish

14. has webbed feet

15. has (two) wings

16. is small (1ittle)

17. Found in zoo

18. found in water
$10(10.53)$

$33(34.74)$

$25 \quad(26.32)$

$1(1.05)$

$24 \quad(25.26)$

$10(10.53)$

$17 \quad(17.89)$

$3 \quad(3.16)$

$7 \quad(7.37)$

$9 \quad(9.47)$

$2(2.11)$

$9 \quad(9.47)$

$10(10.53)$

$3 .(3.16)$

$2 \quad(2.11)$

$3(3.16)$

$10(10.53)$

$9 \quad(9.47)$
Non-essential

72. (75.79)

$19(20.00)$

$23(24.21)$

$44 \quad(46.32)$

$11(11.58)$

$16(16.84)$

$6 \quad(6.32)$

$15(15.79)$

$9 \quad(9.47)$

$6 \quad(6.32)$

$13(13.68)$

$4 \quad(4.21)$

$1 \quad(1.05)$

$10 \quad(10.53)$

$10(10.53)$

$15(15.74)$

$0 \quad(0.00)$

$1 \quad(1.05)$ 
CATESORY 4

FUENITUKF,

\section{Essential}

$26(27.37)$

$22(23.16)$

$20 \quad(21.05)$

3. is confortahel

4. used for sitting on (or at)

$17(17.89)$

$8 \quad(8.42)$

5. has 1-4 legs

6. is supportive (put things on)

7. made of (covered with) cloth

8. a variety of sizes

9. made of plastic

10. made of metal

11. used for decoration

12. a variety of sizes

13. is functional (useful)

14. is various colors

15. many types

16. mane of cushions

17. found in most buildings
$7 \quad(7.37)$

$13(13.68)$

$12(12.63)$

$10(10.53)$

$11(11.58)$

$8 \quad(8.42)$

$8 \quad(8.42)$

$7 \quad(7.37)$

$8 \quad(8.42)$

$5 \quad(5.26)$

8. (8.42)

$6 \quad(6.32)$
Non-essential

$23(24.21)$

$6 \quad(6.32)$

$7(7.37)$

$12(12.63)$

$9(9.47)$

$7 \quad(7.37)$

$3(3.16)$

$1(1.05)$

$2 \quad(2.11)$

$2(2.11)$

$5(5.26)$

$4 \quad(4.21)$

$4(4.21)$

$3(3.16)$

$4 \quad(4.21)$

$2(2.11)$

2 (2.11) 
TABLE

1. has $(1-4)$ legs

2. usea for eating

3. made of wood

4. has flat surface

5. is supportive (put things on)

6. is a piece of furniture

7. is round

8. surrounded by chairs

9. made of metal

10. is square

11. found in kitchen

12. is hard

13. has smooth (finish) top

14. a variety of sizes

15. made of plastic

16. used for sitting on (or at)

17. is a variety od shapes

18. made of glass

19. used for studying

20. is rectangular

\section{Essential}

$11(11.58)$

$46 \quad(48.42)$

$31 \quad(32.63)$

$9 \quad(9.47)$

$22(23.16)$

$8 \quad(8.42)$

$13(13.68)$

$15(15.79)$

$17 \quad(17.89)$

$10 \quad(10.5 .3)$

$12(12.63)$

$5 \quad(5.26)$

$10(10.53)$

$10(10.53)$

$9 \quad(9.47)$

$10 \quad(10.53)$

$6 \quad(5.32)$

$7 \quad(7.37)$

$8 \quad(8.42)$

$7 \quad(7.37)$
Non-essentia 1

$68(71.58)$

$10 \quad(10.53)$

$24(25.26)$

$35(36.84)$

$13(13.68)$

$19(20.00)$

$10(10.53)$

$4(4.21)$

$7 \quad(7.37)$

$8 \quad(9.42)$

$2 \quad(5.11)$

$9 \quad(9.47)$

$4 \quad(4.21)$

$4 \quad(4.21)$

$4 \quad(4.21)$

$1(1.05)$

4 (4.2.1)

$3 \quad(3.16)$

$1(1.05)$

$4 \quad(4.21)$ 
BOOKCASF.

5. found in libraries

6. holds trinkets (ornaments,

$$
\text { candles) }
$$

7. is tall (high)

8. is a piece of furniture

9. found against (close to)

$$
\text { a waIl }
$$

10. is brown

11. holas objects

12. is large (wide)

13. found in study room

14. used in homes
Fssential

$31 \quad(32.63)$

$12(11.43)$

$40 \quad(42.11)$

$17(17.89)$

17 (17.89)

Non-essential

$52(54.74)$

$47(49.47)$

$20(21.05)$

$5 \quad(5.26)$

$3(3.16)$

$10(10.53) \quad 6 \quad(5.32)$

$12(11.43) \quad 2 \quad(2.11)$

$4(4.21) \quad 6 \quad(6.32)$

$9(9.47) \quad 4 \quad(4.21)$

$10(10.53) \quad 3 \quad(3.16)$

$\begin{array}{lll}9 & (9.47) \quad 2 \quad(2.11)\end{array}$

$10(10.53) \quad 0 \quad(0.00)$

$\begin{array}{llll}7 & (7.37) & 1 & (1.05)\end{array}$

$\begin{array}{llll}9(9.47) & 0 & (0.00)\end{array}$ 
STOOL

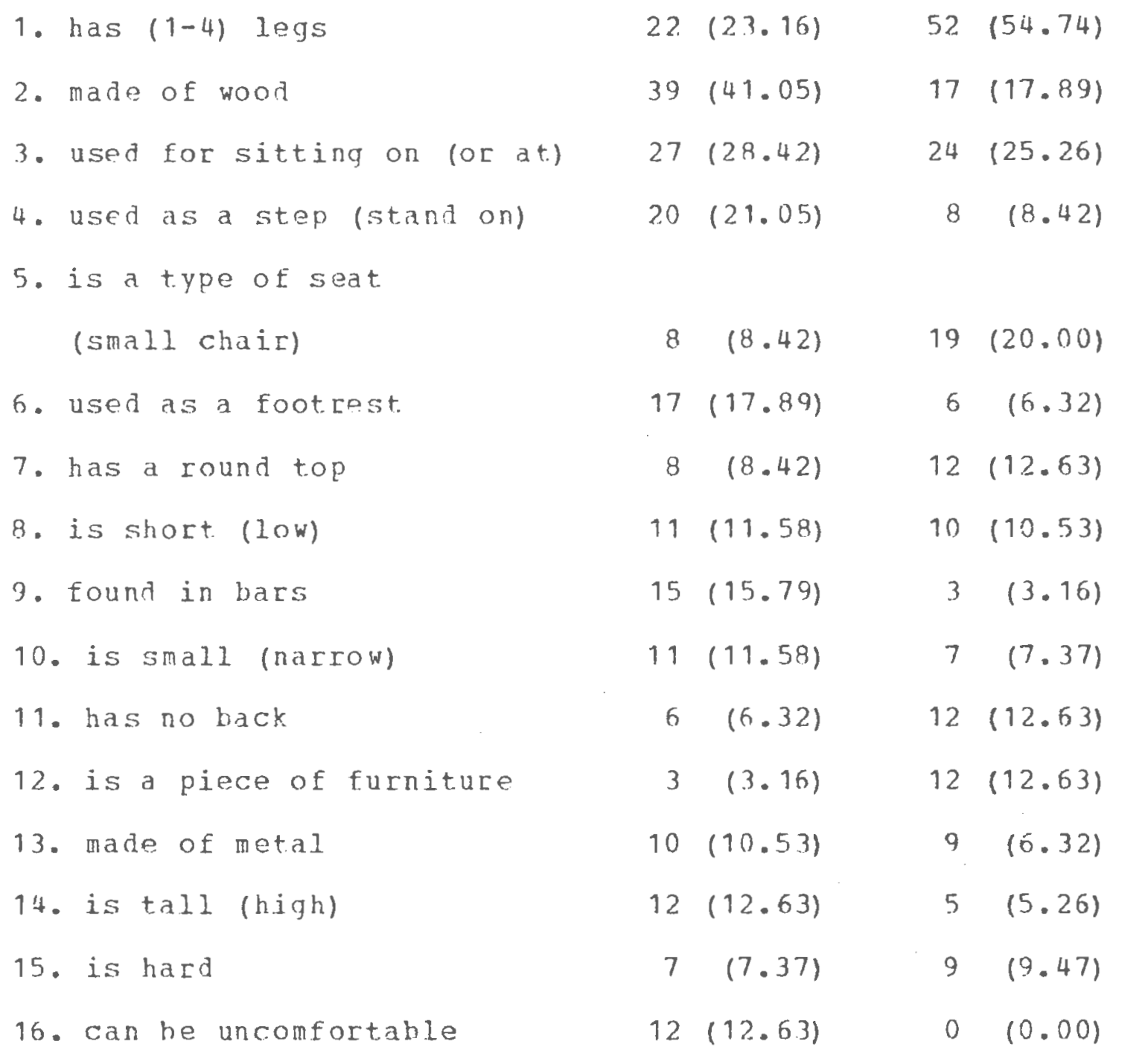


WASTEBASKET

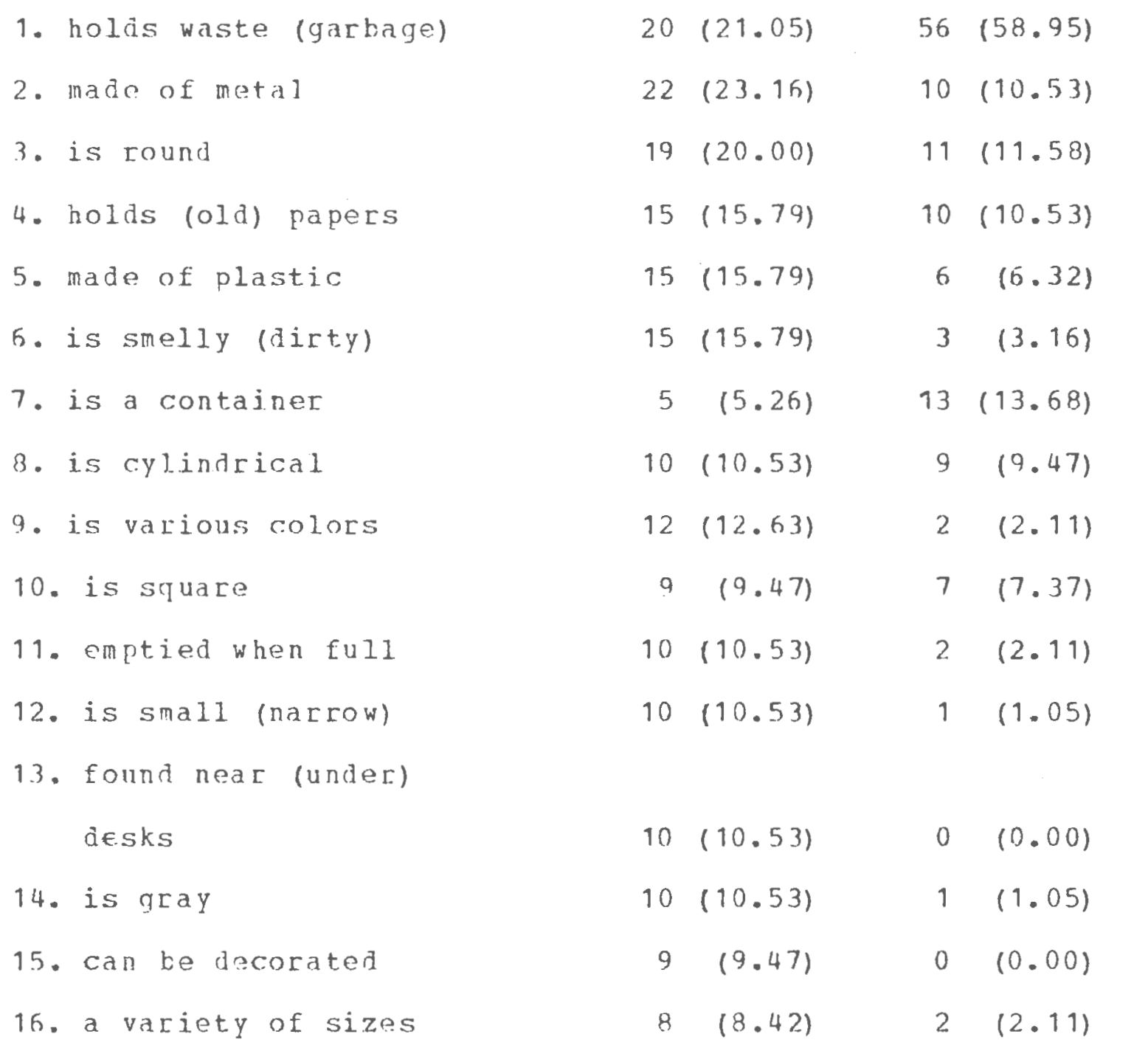

$$
\begin{array}{ll}
\text { Fssential } & \text { Non-essential } \\
20(21.05) & 56(58.95)
\end{array}
$$$$
22(23.16) \quad 10(10.53)
$$$$
19(20.00) \quad 11(11.58)
$$$$
15(15.79) \quad 10(10.53)
$$$$
15(15.79) \quad 6 \quad(6.32)
$$$$
15(15.79) \quad 3 \quad(3.16)
$$$$
5(5.26) \quad 13(13.68)
$$$$
10(10.53) \quad 9 \quad(9.47)
$$$$
12(12.63) \quad 2(2.11)
$$$$
\begin{array}{llll}
9 & (9.47) \quad 7.37)
\end{array}
$$$$
\begin{array}{lll}
0 & (10.53) \quad 2.11)
\end{array}
$$$$
\begin{array}{lll}
0(10.53) & 1 & (1.05)
\end{array}
$$$$
10(10.53) \quad 1 \quad(1.05)
$$$$
\begin{array}{llll}
9(9.47) & 0 & (0.00)
\end{array}
$$$$
8 \quad(8.42) \quad 2 \quad(2.11)
$$ 
page 71

\section{CATEGORY 5 \\ VFHICLE}

\section{Fssential}

1. means of transportation

2. has wheels

3. needs a fare

4. carries people (passengers)

5. has engine (motor)

6. is various colors

7. is various sizes

8. covers distance

9. is driven

10. is various shapes

11. is dangerous (accidents)

12. is fast

13. made of metals

14. has steering wheel

15. is expensive

$\begin{array}{llll}8 & (8.47) & 56 & (58.95) \\ 11 & (11.58) & 38 & (40.00) \\ 10 & (10.53) & 11 & (11.58) \\ 8 & (8.42) & 5 & (5.26) \\ 8 & (6.32) & 7 & (7.37) \\ 9 & (9.47) & 6 & (6.32) \\ 11 & (11.58) & 4 & (4.21) \\ 5 & 5.26 & 10 & (10.53) \\ 4 & (4.21) & 9 & (9.47) \\ 7 & (7.37) & 6 & (5.32) \\ 12 & (12.63) & 0 & (0.00) \\ 8 & (8.42) & 2 & (2.11) \\ 7 & (7.37) & 4 & (4.21) \\ 4 & (4.21) & 7 & (7.37) \\ 8 & (8.42) & 0 & (0.00)\end{array}$


$\mathrm{CAR}$

1. has wheels

2. means of transportation

3. takes qas

4. has engine (motor)

5. is various colors

6. has steering wheel

7. is expensive

8. has (2 to 4) doors

9. is various sizes

10. has glass windows

11. is a vehicle

12. has seats

13. is dangerous (accidents)

14. is various makes

15. is fast

16. is various styles

17. has metalic frame

18. is driven

19. goes on roads (nighways, driveways)

20. has trunk

21. has lights

22. needs repairs

23. has rubber tires

24. is fun for cruising

\section{Essential}

$$
8 \quad(8.42)
$$

$10(10.53)$

$20(21.05)$

$7 \quad(7.37)$

$24(25.26)$

$10 \quad(10.53)$

$22(23.15)$

$9 \quad(9.47)$

$22(2.3 \cdot 16)$

$5 \quad(5.26)$

$5 \quad(5.26)$

$8 \quad(8.42)$

$20 \quad(21.05)$

$14(14.74)$

$15(15.74)$

$5 \quad(5.26)$

$5 \quad(5.26)$

$7 \quad(7.37)$

$8 \quad(8.42)$

$6 \quad(6.32)$

$5 \quad(5.26)$

$12(12.63)$

$1(1.05)$

$10(10.53)$
Non-essential

$57(60.00)$

$37(38.95)$

$19(20.00)$

$29 \quad(30.53)$

$7 \quad(7.37)$

$20 \quad 21.05\}$

$6 \quad(6.32)$

$17(17.89)$

$7 \quad(7.37)$

$19(20.00)$

$19(20.00)$

$6 \quad(6.32)$

$1(1.05)$

$6 \quad(6.32)$

$4 \quad(4.21)$

$19(20.00)$

$12(12.63)$

$8 \quad(8.42)$ 
paqe 73

25. has horn

$7 \quad(7.37)$

$5 \quad(5.26)$

26. has a radio

$8 \quad(8.42)$

$3 \quad(3.16)$

27. has hrakes

$5 \quad(5.26)$

$5 \quad(5.26)$

28. is a symbol of prestiqe (status)

29. is convenient

$$
7 \quad(7.37)
$$

$3 \quad(3.16)$

$7 \quad(7.37)$

$3(3.16)$ 
paqe 74

TROLLEY

\section{Essential}

1. goes on tracks

2. means of transportation

3. Found in san Francisco

4. carries people (passengers)

5. is old-fashioned

6. is train-like

7. has seats

8. run by electricity

9. attached to a wire overhead

10. has wheels

11. found in cities

12. needs a conductor (driver)

1.3. has a bell

14. is noisy

15. has (glass) windows

16. is various makes

17. is open

18. is a vehicle

19. found in Boston

20. qoes on roads (highways. driveways)

21. goes uphills

\begin{tabular}{|c|c|c|c|}
\hline 8 & $(8.42)$ & 50 & 52.631 \\
\hline 21 & $(22.11)$ & 30 & (31. 5 \\
\hline 38 & $(40.00)$ & 8 & $(8.42)$ \\
\hline 15 & $(15.79)$ & 14 & $(14.74)$ \\
\hline 26 & $(27.37)$ & 4 & $(4.21)$ \\
\hline 5 & $(5.26)$ & 20 & $(23.16)$ \\
\hline 10 & $(10.53)$ & 10 & $(10.53)$ \\
\hline 7 & $(7.37)$ & 11 & $(11.58)$ \\
\hline 5 & $(5.26)$ & 13 & $(13.68)$ \\
\hline 4 & $(4.21)$ & 12 & $(12.63)$ \\
\hline 8 & $(8.42)$ & 6 & $(6.32)$ \\
\hline 10 & $(10.53)$ & 5 & $(5.26)$ \\
\hline 8 & $(8.42)$ & 5 & $(5.26)$ \\
\hline 8 & $(8.42)$ & 3 & $(3.16)$ \\
\hline 4 & $(4.21)$ & 9 & $(9.47)$ \\
\hline 10 & $(10.53)$ & 1 & $(1.05)$ \\
\hline 6 & $(6.32)$ & 4 & $(4.21)$ \\
\hline 1 & $(1.05)$ & 9 & $(9.47)$ \\
\hline 9 & $(9.47)$ & 2 & $(2.11)$ \\
\hline
\end{tabular}

$3(3.16)$

$6 \quad(6.32)$

$10(10.53)$

$0 \quad(0.00)$ 
SCOTTER

1. has wheels

2. children's toy

3. has steering handle

4. has engine (motor)

5. means of transportation

6. is red

7. is pusher with one foot

8. is a vehicle

9. made of metal

10. takes gas

11. is fast.

12. is driven

13. has seats

$$
\text { Essential }
$$$$
11(11.58)
$$$$
\text { 32. (33.68) }
$$$$
7 \quad(7.37)
$$$$
19(20.00)
$$$$
10(10.5 .3)
$$$$
18 \quad(18.95)
$$$$
15(15.79)
$$$$
4 \quad(4.21)
$$$$
9 \quad(9.47)
$$$$
7 \quad(7.37)
$$$$
11(11.58)
$$$$
8 \quad(8.42)
$$$$
2(2.11)
$$

Non-essential

$73 \quad(76.84)$

$12(12.63)$

$32 \quad(33.68)$

$12(12.63)$

$13(13.68)$

$4 \quad(4.21)$

$6 \quad(5.32)$

$12(12.63)$

$6 \quad(5.32)$

$5 \quad(5.26)$

$1(1.05)$

2. (2.11)

$8 \quad(8.42)$ 
SKATES

1. has sharp blades

2. has wheels

3. is white

4. has laces

5. used for hockey

6. used on ice

7. usen for sport

8. is shoe like

9. is rlack

10. is $\mathrm{fun}$

11. made of leather

12. means of transportation

13. can glide

\section{Pissential}

$11(11.58)$

$8 \quad(8.42)$

$26 \quad(27.37)$

$9 \quad(9.47)$

$16(16.84)$

$14(14.74)$

$16(16.84)$

$7 \quad(7.37)$

$15(15.79)$

$14(14.74)$

2 (2.11)

$9 \quad(9.47)$

$5 \quad(5.26)$
Non-essential

$58 \quad(61.05)$

$32(33.68)$

$8 \quad(8.42)$

$22(23.16)$

$7 \quad(7.37)$

$13(13.68)$

$4 \quad(4.21)$

$11(11.58)$

$4(4.21)$

$3(3.16)$

$11(11.58)$

$3(93.16)$

$6 \quad(6.32)$ 
features $x$ categories $x$ instances $(2 \times 5 \times 5)$ repeated measures factorial design was performed. All three variables were repeated. Features given for category names were treated as a level of instances. Instances were ordered with category name $\left(C_{n}\right)$ as the first level and instances in a decreasing order of relatedness, with instance 1 ( $I_{7}$ ) being the highly related instance and instance $4\left(I_{4}\right)$, the low related instance.

An F max test showed that the variances were homogeneous $[F \max (50,94)=11.03]$. Means and standard deviations of the analysis are presented in Tables 3, 4, 5, and 6. Graphic presentation of the means of the analysis are presented in Figures 1 and 2.

The analysis yielded a significant effect of features, $F(1,94)$ 9.27, $p<.001$. (See Table 7 for summary table of the analysis.) It was found that characteristic features $\left(\overline{\mathrm{X}}_{2}=3.77\right)$ were more prevalent than defining features $\left(\bar{X}_{2}=3.22\right)$.

A significant effect of number of features given for categories was also found, $F(4,376)=30.83, p<.001$. A Newman-Keuls test (see Table 8) revealed that number of features given for category fruit $\left(\overline{\mathrm{X}}_{2}=3.13\right)$ was significantly less than from features for category vehicle $\left(\bar{X}_{5}=3.68\right)$ and bird $\left(\bar{X}_{3}=4.07\right)$. Features given for category furniture $\left(\bar{X} C_{4}=3.22\right)$ were significantly less than features for vehicle and bird. Lastly, features for category vegetable $\left(\bar{X} C_{j}=3.40\right)$ were significantly less than those for bird.

A significant effect of instances was found, $F(4,376)=35.71$, $p<.001$. A Newman-Keuls test (see Table 9) showed that number of features given for category name $\left(\bar{X}_{n}=3.37\right)$ was significantly less than 


\section{TABLE 3}

Means and Standard Deviations of Defining and Characteristic Features for Categories

\section{Categories}

Fruit

Bird

Furniture

Vehicle
Defining

Feature

Means

SD

Means

SD

Means

SD

Means

SD

Means

SD

3.29

1.80

3.01

1.73

4.28

2.07

1.48

3.42
4.23
3.87

1.97

2.06

3.93

3.68

Sum

3.40

3.13

1.80

4.07

3.22

1.98 
TABLE 4

\begin{abstract}
Means and Standard Deviations for Defining and Characteristic Features for Instances
\end{abstract}

Instances

Defining Characteristic

Feature Feature Sum

Category Name

Means

2.73

4.01

3.37

SD

1.65

2.00

Instance 1

Means

3.70

4.11

3.90

$S D$

1.92

2.02

Instance 2

Means

3.60

4.17

3.88

SD

1.89

2.04

Instance 3

Means

2.78

3.05

2.92

SD

1.66

1.74

Means

3.33

3.51

3.42

Instance 4

SD

1.82

1.87 
TABLE 5

$$
\begin{gathered}
\text { Means and Standard Deviations of Features for } \\
\text { Instances and Categories }
\end{gathered}
$$

\section{$\underline{\text { VEGETABLE }}$ FRUIT BIRD FURNITURE}

$\begin{array}{lllllll}\begin{array}{l}\text { Category } \\ \text { Name }\end{array} & \text { Means } & 3.25 & 3.54 & 4.47 & 2.81 & 2.78 \\ & \text { SD } & 1.80 & 1.19 & 2.11 & 1.68 & 1.67 \\ & & & & & & \\ I_{1} & \text { Means } & 2.87 & 3.49 & 3.80 & 3.88 & 5.47 \\ & \text { SD } & 1.69 & 1.87 & 1.95 & 1.97 & 2.34 \\ & & & & & & \\ I_{2} & \text { Means } & 4.38 & 4.76 & 3.90 & 2.95 & 3.43 \\ & \text { SD } & 2.09 & 2.18 & 1.97 & 1.72 & 1.85 \\ I_{3} & \text { Means } & 2.66 & 1.35 & 4.17 & 3.25 & 3.15 \\ & \text { SD } & 1.63 & 1.16 & 2.04 & 1.80 & 1.77 \\ I_{4} & \text { Means } & 3.83 & 2.49 & 4.02 & 3.19 & 3.57\end{array}$




\section{TABLE 6}

Means and Standard Deviations for Defining and Characteristic Features for Categories and Instances

$$
\text { Defining Features }\left(F_{1}\right)
$$

VEGETABLE

$$
\underline{C_{n}} \stackrel{I_{1}}{\underline{I_{2}}} \stackrel{I_{3}}{\underline{I_{4}}} \quad \underline{C_{n}} \stackrel{I_{1}}{\underline{I_{2}}} \stackrel{I_{3}}{\underline{I_{4}}}
$$

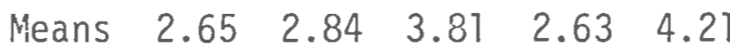

$\begin{array}{lllll}2.67 & 3.45 & 4.75 & 1.47 & 2.69\end{array}$

SD

$\begin{array}{lllll}2.22 & 1.19 & 1.91 & 1.56 & 2.09\end{array}$

$\begin{array}{lllll}2.39 & 1.75 & 1.86 & 1.46 & 1.54\end{array}$

BIRD

$$
\underline{C_{n}} \stackrel{I_{1}}{I_{2}} \quad I_{3} \quad I_{4} \quad C_{n} \quad I_{1} \underline{I_{2}} \quad I_{3} \quad I_{4}
$$



SD

$\begin{array}{lllll}2.28 & 2.04 & 2.51 & 2.93 & 2.40\end{array}$
FURNITURE

$$
\begin{array}{lllll}
1.70 & 2.02 & 1.84 & 1.73 & 1.68
\end{array}
$$

\section{VEHICLE}

$\underline{C_{n}} \stackrel{I_{1}}{I_{2}} \underline{I_{3}} \underline{I_{4}}$



SD $\quad \begin{array}{lllll}2.34 & 3.86 & 1.94 & 1.67 & 2.06\end{array}$ 
TABLE 6 (Continued)

Characteristic Features $\left(F_{2}\right)$

VEGETABLE

$\underline{\text { FRUIT }}$

$\underline{\mathrm{C}_{\mathrm{n}}} \stackrel{\mathrm{I}_{1}}{\mathrm{I}_{2}} \underline{\mathrm{I}_{3}} \stackrel{\mathrm{I}_{4} \quad \mathrm{C}_{\mathrm{n}}}{\underline{\mathrm{I}_{1}}} \underline{\mathrm{I}_{2}} \stackrel{\mathrm{I}_{3}}{\mathrm{I}_{4}}$

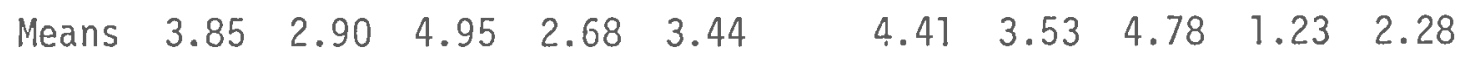

$\begin{array}{lllllllllll}\text { SD } & 2.75 & 1.71 & 3.06 & 2.06 & 2.62 & 3.31 & 2.51 & 2.82 & 1.75 & 2.14\end{array}$

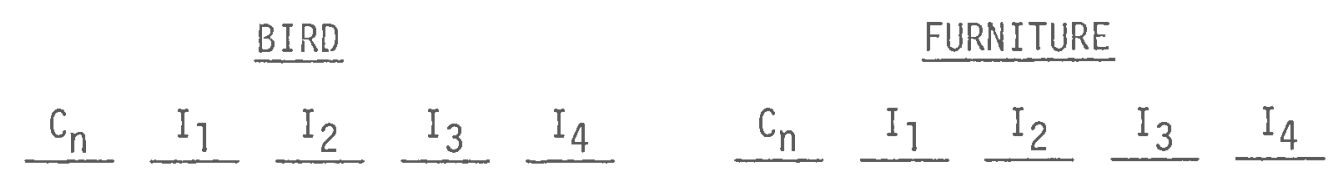

$\begin{array}{lllllllllll}\text { Means } & 4.78 & 3.40 & 3.67 & 3.84 & 3.65 & 4.08 & 4.95 & 3.77 & 4.07 & 4.29\end{array}$

$\begin{array}{lllllllllll}\text { SD } & 2.98 & 2.49 & 2.46 & 2.73 & 2.33 & 2.96 & 3.01 & 2.77 & 2.45 & 2.86\end{array}$

VEHICLE

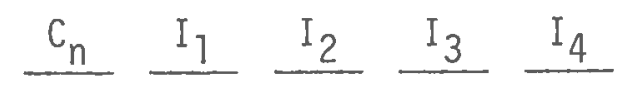

$\begin{array}{llllll}\text { Means } & 2.94 & 5.76 & 3.66 & 3.44 & 3.87\end{array}$

$\begin{array}{llllll}\text { SD } & 2.81 & 3.94 & 2.29 & 2.52 & 2.77\end{array}$ 




Figure 1. Mean number of defining features given for levels of relatedness (i.e., category name and each of the four instances) for the 5 categories. 


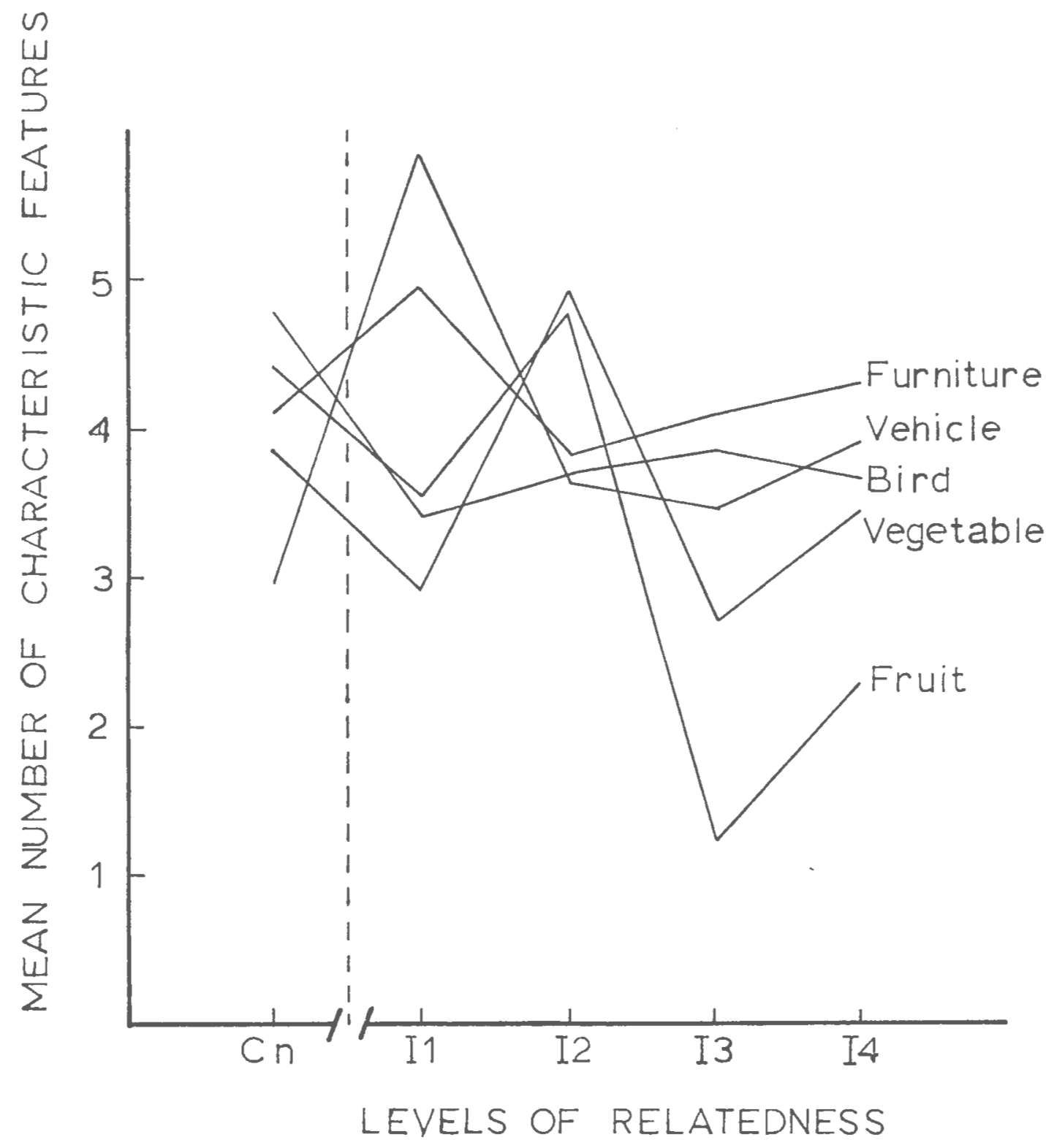

Figure 2. Mean number of characteristic features given for levels of relatedness (i.e., category name and each of the four instances) for the 5 categories. 


\section{TABLE 7}

Summary Table of Analysis of Variance for Number of Defining and Characteristic Features for Categories and Instances

\begin{tabular}{|c|c|c|c|c|c|}
\hline Source & SS & $d f$ & MS & $\mathrm{F}$ & $p$ \\
\hline Subjects & 4873.15 & 94 & 51.84 & & \\
\hline Features & 347.63 & 1 & 347.63 & 9.27 & $<.001$ \\
\hline SF & 3525.21 & 94 & 97.50 & & \\
\hline Categories & 562.00 & 4 & 140.50 & 30.83 & $<.007$ \\
\hline SC & 1713.65 & 376 & 4.56 & & \\
\hline Instances & 640.17 & 4 & 160.03 & 35.71 & $<.001$ \\
\hline SI & 1684.74 & 376 & 4.48 & & \\
\hline $\mathrm{FC}$ & 774.67 & 4 & 193.67 & 25.95 & $<.007$ \\
\hline SFC & 2305.90 & 376 & 7.4 .6 & & \\
\hline FI & 183.08 & 4 & 45.77 & 10.48 & $<.001$ \\
\hline SFI & 1641.64 & 376 & 4.37 & & \\
\hline CI & 1990.60 & 16 & 124.91 & 37.91 & $<.001$ \\
\hline SCI & 4935.98 & 1504 & 3.28 & & \\
\hline FCI & 185.87 & 16 & 71.62 & 2.82 & $<.007$ \\
\hline SFCI & 6188.97 & 1504 & 4.11 & & \\
\hline Tota 1 & 32053.20 & 4749 & & & \\
\hline
\end{tabular}




\section{TABLE 8}

Newman-Keuls Test of Features for Categories

$\begin{array}{llllllll} & \text { Fruit } & \text { Furn. Veget. Veh. } & \text { Bird } \\ \text { Means } & 3.13 & 3.22 & 3.40 & 3.68 & 4.07 & \text { Sc } 9.99 & (r, 376)\end{array}$

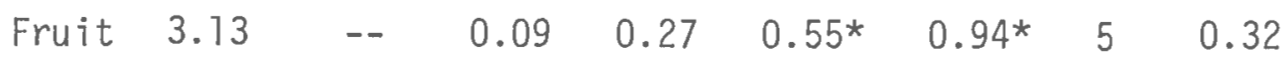

Furn. $3.22 \quad--\quad 0.18 \quad 0.46^{*} \quad 0.85^{*} \quad 4 \quad 0.30$

$\begin{array}{lllllll}\text { Veget. } 3.40 & - & 0.28 & 0.67^{\star} & 3 & 0.28\end{array}$

$\begin{array}{llllll}\text { Veh. } 3.68 & - & 0.39 & 2 & 0.25\end{array}$

Bird $\quad 4.07$

*indicates significant mean pairs 


\section{TABLE 9}

Newman-Keuls Test for Features at Instances

$$
\mathrm{I}_{3} \quad \mathrm{C}_{\mathrm{n}} \quad \mathrm{I}_{4} \quad \mathrm{I}_{2} \quad \mathrm{I}_{7}
$$

Means $2.92 \quad 3.37 \quad 3.42 \quad 3.88 \quad 3.90 \quad r \quad S \bar{I} 9.99(r, 376)$

$I_{3} 2.92 \quad--\quad 0.45 * \quad 0.50 * 0.96 * 0.98 * 50.27$

$\begin{array}{llllllll}C_{n} & 3.37 & -- & 0.05 & 0.51 * & 0.53 * & 4 & 0.25\end{array}$



$\begin{array}{llllll}\mathrm{I}_{2} & 3.88 & -- & 0.02 & 2 & 0.19\end{array}$

I) 3.90

*indicates significant mean pairs 
features for Instance $2\left(\bar{X}_{2}=3.88\right)$ and Instance $1\left(\bar{X}_{1}=3.90\right)$. Number of features given for Instance $3\left(\bar{X}_{3}=2.92\right)$ was less than features given for category name and a 11 instances. Features for Instance 4, the low related instance $\left(\bar{X}_{4}=3.42\right)$, were found to be significantly less than features given for Instance 2 and 1 , the high related instances.

Number of defining and characteristic features given for different categories, Features $X$ Categories interaction, yielded a significant effect, $F(4,376)=25.95, p<.001$. Subsequent simple main effects test for defining and characteristic features given for different categories (see Table 10) found that only for category furniture there were more characteristic features given than defining, $F(4,376)=$ 32.84, $p<.001$. The respective means for the category furniture were: $\bar{X} F_{7} C_{4}=2.20$ and $\bar{X} F_{2} C_{4}=4.23$. Defining features given at different categories were found to be significant, $F(4.376)=35.75, p<.001$. Similarly, characteristic features given for different categories were found to be significant, $F(4.376)=9.04, p<.001$.

A significant effect was found for number of defining and of characteristic features given for category name and instances, features $X$ instances interaction $[F(4,376)=10.48, p<.001]$. Subsequent simple main effects test of overa 17 features given for category name and instances (see Table 11) revealed that for category name $[F(4,376)=$ 22.26, $\mathrm{p}<.001]$, Instance $1[\mathrm{~F}(4,376)=2.28, \mathrm{p}<.001]$ and Instance 2 $[F(4,376)=4.41, p<.001]$ there were more characteristic features given than defining (see Table 4 for appropriate means). Simple main effects test for defining and characteristic features given for 
TABLE 10

Sumnary Table for Partitioning of Features $X$ Categories Interaction

F at levels of $C$

\begin{tabular}{|c|c|c|c|c|c|}
\hline Source & SS & $d f$ & MS & $F$ & $P$ \\
\hline FC & 774.67 & 4 & 193.67 & 25.95 & $<.001$ \\
\hline $\mathrm{F}$ at $\mathrm{C}_{1}$ & 26.95 & 1 & 6.73 & 0.90 & \\
\hline $\mathrm{F}$ at $\mathrm{C}_{2}$ & 13.44 & 1 & 3.36 & 0.45 & \\
\hline $\mathrm{F}$ at $\mathrm{C}_{3}$ & 40.03 & 1 & 10.00 & 1.34 & \\
\hline $\mathrm{F}$ at $\mathrm{C}_{4}$ & 980.24 & 1 & 254.06 & 32.84 & $<.001$ \\
\hline$F$ at $C_{5}$ & 61.65 & 1 & 15.41 & 2.06 & \\
\hline SFC & 2805.90 & 376 & 7.46 & & \\
\hline \multicolumn{6}{|c|}{$\mathrm{C}$ at levels of $\mathrm{F}$} \\
\hline FC & 774.67 & 4 & 193.67 & 25.95 & $<.001$ \\
\hline$C$ at $F_{1}$ & 1066.69 & 4 & 266.67 & 35.75 & $<.001$ \\
\hline$C$ at $F_{2}$ & 269.68 & 4 & 67.42 & 9.04 & $<.001$ \\
\hline SFC & 2805.90 & 376 & 7.46 & & \\
\hline
\end{tabular}


TABLE 11

Summary Table for Partitioning of Features $X$ Instances Interaction

F at levels of I

Source

FI

$F$ at $C_{n}$

$F$ at $I_{1}$

$F$ at $I_{2}$

$\mathrm{F}$ at $\mathrm{I}_{3}$

$F$ at $\mathrm{I}_{4}$

SFI

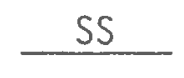

183.08

389.12

39.92

77.16

17.31

5.58

1641.64

I at levels of $\mathrm{F}$

FI

183.08

$I$ at $F_{1}$

389.63

I at $F_{2}$

436.62

SF I

1641.64
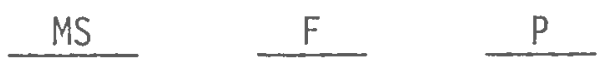

$\underline{d f}$

4

45.77

10.48

$<.001$

97.28

22.26

$<.001$

9.98

2.28

4.41

19.29

4.32

0.98

1.39

0.31

4.37 
category name and instances (see Table 11) showed that characteristic features given for instances and category name were significantly different from each other, $F(4,376)=24.97, p<.001$. Defining features for instances and category names were also found to be significantly different, $F(4.376)=22.28, p<.001$. In fact, defining features given for category names $\left(\overline{X F}_{1} C_{n}=2.73\right)$ were less than defining features for Instance $1\left(\overline{\mathrm{XF}}_{1} \mathrm{I}_{1}=3.70\right)$, Instance $2\left(\overline{\mathrm{XF}}_{7} \mathrm{I}_{2}=3.60\right)$ and Instance 4 $\left(\overline{X F}_{7} I_{4}=3.33\right.$ ), but not from Instance $3\left(\bar{X}_{7} I_{3}=2.78\right)$ (see Table 12 for Newman-Keuls test).

Finally, the analysis yielded a significant features $X$ categories $X$ instances interaction, $F(16,1504)=2.82, p<.001$. Subsequent simple main effects test (see Table 13) showed that number of defining features given at categories $X$ instances were significantly different, $F(16,1504)=12.86, p<.001$, as well as number of characteristic features given at categories $X$ instances, $F(16,1504)=20.17, p<.001$ (see Table 5 for appropriate means). A subsequent simple main effects test (see Table 13) showed that number of defining features given for a level of relatedness for different categories were significantly different. Similarly, characteristic features given for a level of relatedness for different categories were found to be significant. Furthermore, defining features given for different categories and their instances were found to be significantly different except for the bird category (see Table 13 continued). Newman-Keuls tests were performed on the significant differences to see whether the number of defining features given for the category name of a specific category was significantly less than the number of defining features given for the 


\section{TABLE 12}

Newman-Keuls Test for Defining

Features at Instances

$\begin{array}{lllll}\mathrm{C}_{\mathrm{n}} & \mathrm{I}_{3} & \mathrm{I}_{4} & \mathrm{I}_{2} & \mathrm{I}_{1}\end{array}$

Means $2.73 \quad 2.78 \quad 3.33 \quad 3.60 \quad 3.70 \quad r \quad S r$ at $F_{1} .99(r, 376)$

$\begin{array}{lllllllll}C_{n} & 2.73 & - & 0.05 & 0.60 * & 0.87 * & 0.97 * & 5 & 0.44\end{array}$

$\begin{array}{llllllll}\mathrm{I}_{3} & 2.78 & -- & 0.55 * & 0.82 * & 0.92 * & 4 & 0.42\end{array}$

$\begin{array}{lllllll}\mathrm{I}_{4} & 3.33 & -- & 0.27 & 0.37 * & 3 & 0.39\end{array}$

$\begin{array}{llllll}I_{2} & 3.60 & -- & 0.10 & 2 & 0.35\end{array}$

$\mathrm{I}_{1} \quad 3.70$

*indicates significant mean pairs 
TABLE 13

Summary Table for Partitioning of Features $X$ Categories $X$ Instances Interaction

\section{CI at levels of $F$}

Source

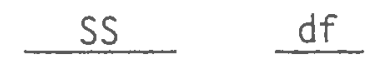

FCI

CI at $F_{T}$

$C I$ at $F_{2}$

SFCI
185.87

847.20

1329.22

6188.97

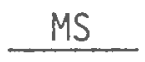

11.62

52.95

83.08

4.12

C at levels of FI

$\begin{array}{lrrrrr}\text { FCI } & 185.87 & 16 & 11.62 & 2.82 & <.001 \\ \mathrm{C} \text { at } \mathrm{F}_{1} \mathrm{C}_{n} & 333.80 & 4 & 83.45 & 20.26 & <.001 \\ \mathrm{C} \text { at } \mathrm{F}_{1} \mathrm{I}_{1} & 381.67 & 4 & 95.42 & 23.17 & <.001 \\ \mathrm{C} \text { at } \mathrm{F}_{1} \mathrm{I}_{2} & 374.97 & 4 & 93.74 & 22.76 & <.001 \\ \mathrm{C} \text { at } \mathrm{F}_{1} \mathrm{I}_{3} & 459.87 & 4 & 114.97 & 27.91 & <.001 \\ \mathrm{C} \text { at } \mathrm{F}_{1} \mathrm{I}_{4} & 363.88 & 4 & 90.97 & 22.09 & <.001 \\ \mathrm{C} \text { at } \mathrm{F}_{2} \mathrm{C}_{n} & 183.69 & 4 & 45.92 & 11.15 & <.001 \\ \mathrm{C} \text { at } \mathrm{F}_{2} \mathrm{I}_{1} & 542.72 & 4 & 135.68 & 32.94 & <.001 \\ \mathrm{C} \text { at } \mathrm{F}_{2} \mathrm{I}_{2} & 155.75 & 4 & 38.94 & 9.45 & <.001 \\ \mathrm{C} \text { at } \mathrm{F}_{2} \mathrm{I}_{3} & 500.59 & 4 & 125.15 & 30.38 & <.001 \\ \mathrm{C} \text { at } \mathrm{F}_{2} \mathrm{I}_{4} & 216.18 & 4 & 54.04 & 13.12 & <.001 \\ \text { SFC } & 6188.87 & 1504 & 4.12 & & \end{array}$


TABLE 13 (Continued)

I at levels of FC

\begin{tabular}{|c|c|c|c|c|c|}
\hline Source & SS & $d f$ & MS & $F$ & $\mathrm{P}$ \\
\hline FCI & 185.87 & 16 & 11.62 & 2.82 & $<.001$ \\
\hline I at $F_{1} C_{1}$ & 203.80 & 4 & 50.95 & 12.36 & $<.001$ \\
\hline I at $F_{1} C_{2}$ & 552.01 & 4 & 138.00 & 34.49 & $<.001$ \\
\hline I at $F_{7} C_{3}$ & 9.30 & 4 & 2.32 & 0.56 & \\
\hline I at $\mathrm{F}_{7} \mathrm{C}_{4}$ & 83.98 & 4 & 30.99 & 5.09 & $<.001$ \\
\hline I at $F_{7} C_{5}$ & 392.02 & 4 & 98.00 & 23.78 & $<.001$ \\
\hline I at $\mathrm{F}_{2} \mathrm{C}_{1}$ & 307.85 & 4 & 76.96 & 18.68 & $<.001$ \\
\hline I at $\mathrm{F}_{2} \mathrm{C}_{2}$ & 834.68 & 4 & 208.67 & 50.64 & $<.001$ \\
\hline I at $F_{2} C_{3}$ & 108.13 & 4 & 27.03 & 6.56 & $<.001$ \\
\hline I at $\mathrm{F}_{2} \mathrm{C}_{4}$ & 74.26 & 4 & 18.56 & 4.50 & $<.001$ \\
\hline I at $F_{2} C_{5}$ & 441.32 & 4 & 110.33 & 26.78 & $<.001$ \\
\hline SFCI & 6188.97 & 1504 & 4.12 & & \\
\hline
\end{tabular}


instances of that category (see tables 15-18). The above (inequality) relation held only for the following cases: (a) category "vegetable" for $\mathrm{I}_{2}$ and $\mathrm{I}_{4}$, (b) category "fruit" for $\mathrm{I}_{2}$, (c) category "furniture" for $I_{1}$, and (d) category "vehicle" for $I_{7}$.

To further investigate whether defining features are central to word meanings a chi-square test was performed on the first response given for category names and instances to see whether defining features, characteristic features or other idiosyncratic responses were more frequently given (see Table 19). The result of the chi-square showed that defining features were more frequently given first than would be expected by chance $\left(x^{2}=1,091.21, d f=2, p<.001\right)$. The first feature given for instances was also compared against the first feature given for categories (see Table 20) and it was found that defining features were more frequently given first for instances than for categories $\left(x^{2}=115.64, d f=2, p<.001\right)$. Lastly, for all levels of relatedness defining features were more frequently given first to instances than were given to the category names (see Table 21). 
TABLE 14

PERCENTAGES OF FEATURES FROM RECOGNITION MEMORY

\section{CATEGORY 1}

VEGETABLE ( N=157)

Fssential Non-essential Not a property
1. is various colors 62.9
35.3
0.6
2. is a herb
16.2
56.9
25.7
3. has yellow flowers 4.2
83.2
11.4
4. is red
6.6
86.8
5.4
5. has green stem
31.7
65.3
1.8
6. has fuzzy seeds
6.6
83.8
8.4
7. has faible leaves
19.2
73.7
6.0
8. is a feuit
9.0
26.9
$62.3 *$
9. grows from soil
74.9
21.6
2.4
10. can be cultivated 66.5
29.3
3.0
11. is a spice
6.6
64.1
25.7
12. hasi green leaves 28.7
66.5
1.8
13. can be cooked
52.1
45.5
0.6
14. grows in gardens
54.5
43.7
0.6
15. is a food
84.4
14.4
0.0
16. makes sauce
13.8
75.4
9.0
17. tastes goor
18.6
77.2
3. 0

Footnote: * indicates statistical significance $(p<.05)$ between the distributions of essential. non-essential and not a property categorjes when category names were presented 
prior and after instances.

19. used for decorating

food

19. can be dried up

20. tastes bad

21. eaten in salads

22. is a weed

23. is green

24. grows in lawns

25. has yellow petals

26. grows in grass

4.2

27. made into pie

6.6

28. is nutritious

$$
\text { (healthy) }
$$

70.7

29. is mushy

5.4

30. is many varieties

68.3

31. is small

32. made into wine

33. remover from lawns

$$
\text { (not liked) }
$$

4. 2

5.4

1.8

5.4

34. is hard

8.4

35. is yellow

7.8

36. is a flower

37. has stem

33.5

38. grows in soil

$$
\text { (ground) }
$$

64.7

39. used for cooking
85.0

7.8

76.0

3.0

82.6

15.0

74.3

1.8

45.5

49.7

83.2

3.6

52.1

42.5

68.9

2.5 .7

59.9

34.1 *

12.6

79.0

0.6

25.9

3.6

29.3

0.6

89.8

4.8

58.7

34.7

46.7

49.7

84.4

9.0

83.2

7.2

51.5

38.9 *

61.7

3.6

33.5

6.6

54.5

1.8 
40. eaten at meals

55.7

41. tastes bitter

42. eaten fresh

43. has vitamins

44. is juicy

45. is skin-covered

46. is pretty

47. has many shapes

48. is almost round

49. children do not

28.1

72.5

12.6

29.3

1.8

50.9

3.6

$$
3.6
$$

1 ike it

50. is a plant

51. has lacy leaves

52. grows in spring

53. is a wild flower
42.5

89.8

70.1

25.1

82.6

65.3

84.4

43.1

84.4

74.9

29.3

84.9

71.3

49.7

54. has white fluff.

(attached to seeds) 2.5

55. used for seasoning

19. 2

1.8

7.8

4.8

44.3

34.1

$14 \cdot 4$

1. 8

56. is edible

78.4

19.2

57. blown away

$$
\text { (for good luck) } \quad 1.2
$$

33.5

64.1

58. is shiny

1.2

86.8

10.8

59. is short.

1.8

88.6

8.4

60. contains acia

51.6

77.8

8. 4

61. has 1ittle or

no taste

4.8

73.7

20.4 
62. grows in vines

12.6

71.9

14.4

63. has minerals
(iron)
50.9
45.5
2.4

64. qrows in the sumer

29.3

25.7

31.1
67.1

1.8

65. eaten raw

66. grows in fields
71.9

1.2

60.5

6.0 


\section{SEINACH $(N=90)$}

Fssential Non-essential Not a property

1. is various colors 10.0

2. is a herb

3. has yellow Elowers

4. is red

5. has green stem

6. has fuzzy seeds

7. has edible leaves 70.0

8. is a fruit 1.1

9. grows froin soj.l 75.6

10. can be cultivater 50.0

11. is a spice

12. has green leaves

13. can be cooked

14. grows in gardens 52.2

15. is a food

16. makes sauce

90.0

17. tastes good

18. used for decorating
11.1

12.2

3.3

22.2

4.4

84.4

5.6
30.0

2.2. 2

26.7

8.9

23.3

26.7

23.3

13.3

18.9

38.9

16.7

14.4

45.6

44.4

6.7

38.9

65.6

62.2

53.3

65.6

65.6

20.0

8.9

35.6
56.7

$54.48 \%$

61.1

84.4 *

10.0

61.1

3.3

82.2

3.3

8.9

73.3

0.0

2. 2

1.1

1. 1

52.2

8.9 *

24. grows in lawns

35.6

2.3 .3

32.2

28.9

10.0

72.2

4.4

56.7 
25. has yellow petals 7.8

26. qrows in lawns

6.7

27. made into pie

14.4

2.8. is nutritious

(healthy)

78.9

2.9. is mushy

$15 \cdot 6$

30. is many varieties 25.6

31. is small

6.7

32. made into wine

1.1

33. removed from lawns

$$
\text { (not liked) }
$$

$3 \cdot 3$

34. is hard

35. is yellow

36. is a flower

$5 \cdot 6$

37. has stem

50.0

38. grows in soil

$$
\text { (ground) }
$$

65.6

39. used for cooking

41.1

40. eaten at meals

53.3

41. tastes hitter

4.4

42. eaten fresh

23.3

43. has vitamins

44. is juicy

45. is skin-coverd

46. is pretty

47. has many shapes

48. is almost round

78.9

12.2

$8 \cdot 9$

$13 \cdot 3$

26.7

$4 \cdot 4$
14.4

40.0

51.7

16.7

6.3 .3

$75.6 *$

0.0

35.6

27.8

18.9

77.8

18.9

70.0

31.1

64.4

17.8

$75.6 *$

17.8

74.4

2.7 .8

10.0

5.6

52.2

4.4

43.3

1. 1

71.1

2.2 .2

63.3

11. 1

15.6

$2.2 *$

43.3

42.2

18.9

70.0

65.6

18.9

34.4

36.7

33.3

60.0 
49. chilaren do not

like it $\quad 15.6$

50. is a plant

77.8

51. has lacy laaves

37.8

52. qrows in spring

27.8

53. is a wild flower

8.9

54. has white fluff

$$
\text { (attached to seeds) } 5.6
$$

55. used for seasoning 8.9

56. is edihle

82.2

57. hlown away

(for good luck)

$$
2.2
$$

61. has little or

no taste

62. grows in vines

63. has minerals

64. grows in the

$$
\text { summer }
$$

65. eaten raw

66. grows in fields

67 . is a vegetahle
34.4

7. 8

10.0

7.8

4.4

76.7

30.0

$14 \cdot 4$

30.0

92.2
15.6

50.0

65.6

37.8

67.8

14.4

13.3

$5.6 \%$

28.9

7.8

64.4 (

23.3

67.8

33.3

0.0

15.6

78.9

40.0

21.1

24.4

40.0

72.2

2. 2

18.9

11. 1

13.3

15.6

4.4

1. 1 


$$
\operatorname{TOMATO}(\mathrm{N}=77)
$$

Fssential Non-essential Not a property

1. is various colors 15.6

2. is a herb

3. has yollow flowers 13.0

4. is red

5. has green stem 59.7

6. has fuzzy seeds 2.6

7. has edible leaves 2.6

8. is a fruit

9. grows Erom soil

10. can be cultivated 54.5

11. is a spice

12. has green leaves 57.1

13. can be cooker 50.6

14. grows in gardens 54.5

15. is a food

16. makes sauce

17. tastes good

18. used for decorating

$$
\text { food }
$$

19. can he dried up

20. is many varieties

21. eaten in salads

22. is a weed

23. is green

24. grows in lawns

$$
10.4
$$$$
2.6
$$$$
0.0
$$

45.5

0.0

13.0

5.2
45.5

37.7

24.7

22.1

18.?

35.1

19.5

31.5

19.5

19.5

40.3

22. 1

36.4

48.1

39.0

0.0

70.1

62.2

1. 3

5.2

75.3

59.7

36.4

6.5

3.9

70.1

3.9

1.3

2.6

0.0

49.4

2.6

70.1

6.5

68.8

20.8

26.0

67.5

50.6

49.4

1. 3

49.4

89.6

9.1

24.7

62.3

58.4 
25. has yellow petals 10.4

26. qrows in grass

1.3

27. made into pie

2.6

28. is nutritious

$$
\text { (healthy) }
$$

29. is mushy

71.4

24.7

30. is many varieties

31. is 5 mall

32. made into wine

33. cemoved from lawns

$$
\text { (not liked) }
$$

0.0

34. is hard

2.6

6.5

36. is a flower

37. has stem

5. ?

61.0

38. grows in soil

(q cound)

68.8

44.2

39. usen for cooking

46.8

$2 \cdot 6$

51.9

79.2

66.2

72.7

9.1

26.0

64.9

47. has many shapes
35. is yellow

26.0

29.9

32.5

62.3

66.2

63.6

24.7

66.2

40.3

71.4

19.5

2.6 *

9.1

10.4

$7.8 *$

77.9

15.6

83.1

28.6

68.8

32.5

5.5 .8

24.7

68.8

28.6

10.4

18. 2

54.5

1.3

46.8

5.2

44.2

53.2

48.1

0.0

20.8

0.0 *

28.6

5. 2

22.1

5.2

67.5

22. 1

40.3

33.8

32.5

0.0 
49. children do not.

$$
\text { like it }
$$

50. is a plant

51. has lacy leaves

52. grows in spring

53. is a wild flower

2.6

54. has white flutf

(attached to seeds) 0.0

55. used for seasoning 22. 1

56. is edihle

84.4

57. blown away

$$
\text { (for good luck) }
$$

1.3

58. is shiny

28.6

3.9

59. is short

60. contains acid

66.2

61. has little or

no taste

3. 9

39.0

58.4

48.1

45.5

31.2

61.0

67. is a vegetable

68. grows in the spring

66. grows in fields

$$
68.8
$$

28.6

$$
14.3
$$$$
5.2
$$

36.4

51.9

50.6

19.5

16.9

$80.5 *$

11.1

87.0

55.8

22.1

13.0

1.3

7.8

89.6

59.7

10.4

37.7

55.8

19.5

9.1

65. eaten raw

40.3

54.5

24.7

36.4

$27 \cdot 3$

$11.7 *$

45.5

6.5

50.6

2.6

45.5

20.8

15.6

22. 1

45.5 20.8 


$$
\text { PARSLFY }(N=90)
$$

Essential Non-essential Not a property

\begin{tabular}{|c|c|c|c|}
\hline 1. is various colors & 3.3 & 37.8 & 56.7 \\
\hline . is a herb & 78.9 & 15.6 & 4.4 \\
\hline 3. has yellow flowers & 4.4 & 28.9 & 64.4 \\
\hline 4. is red & $2 \cdot 2$ & 13.3 & 83.3 \\
\hline - has green stem & 70.0 & 2.3 .3 & 4.4 \\
\hline 6. has Euzzy seeds & 4.4 & 28.9 & 65.6 \\
\hline 7. has edible leaves & 74.4 & 22.2 & 3.3 \\
\hline 8. is a fruit & 4.4 & $13 \cdot 3$ & 82.2 \\
\hline 9. grows from soil & 67.8 & 27.8 & 3.3 \\
\hline 10. can be cultivatei & 40.0 & 52.2 & 7.8 \\
\hline 11. is a spice & 54.4 & 27.8 & 17.8 \\
\hline 12. has green leaves & 72.2 & 20.0 & 7.8 \\
\hline 13. can he cooked & 42.2 & 55.6 & 2.2 \\
\hline 14. grows in garden:s & 52.2 & 47.8 & 0.0 \\
\hline 15. is a food & 65.6 & 27.8 & 5.6 \\
\hline 16. makes sauce & 7.8 & 56.7 & 34.4 \\
\hline 7. tastes goor & $12 \cdot 2$ & 78.9 & 8.9 \\
\hline
\end{tabular}

19. used for decorating

$\begin{array}{llll}\text { food } & 44.4 & 52.2 & 3.3\end{array}$

19. can be dried up 48.9

$42.2 \quad 7.8$

20. tastes bad

$3 \cdot 3$

71.1

25.6

21. edten in salads 36.7

$62.2 \quad 1.1$

22. is a wecd

3.3

25.6

$71.1 *$

23. is green

81. 1

16.7

1. 1

24. grows in lawns

4.6

35.6

56.7 
25. has yellow petals 2.2

26. grows in grass

10.0

1.1

27. made into pic

28. is nutritious

$$
\text { (healthy) }
$$

45.6

2.2

29. is mushy

30. is many varieties

34.4

31. is small

43.3

32. made into wine

33. removed from lawns

$$
\text { (not liked) }
$$

$3 \cdot 3$

1.1

1. 1

7.8

71.1

37. has stem

38. grows in soil

(ground)

39. used for cooking

64.4

47.8

40. eaten at meals

27.8

7.8

41. tastes bitter

36.7

52.2.

5.6

5.6

2.2

48. is almost round

49. children do not

$$
20.0
$$

42.2

23.3

48.9

25.6

45.6

50.0

21.1

34.4

61.1

15.7

82.2

27.8

70.0

36.7

55.6

26.7

1. 1

30.0

4.4

51.1

1. 1

70.0

2.2

53.3

38.9

57.8

5.6

44.4

3.3

35.6

58.9

15.6

77.8

24.4

73.3 
50. is a plant 77.8

51. has 1acy leaves 73.3

52. grows in spring 21.1

53. is a wild flower 4.4

54. has white Eluff

$$
\text { (attached to seers) } 1.1
$$

55. used for seasoning 63.3

56. is edible

82.2

57. blown away

(for good luck)

1. 1

4.4

42.2

11.1

60. contains acid

61. has little or

no taste

22.2

4.4

62. grows in vines

54.4

63. has minerals

64. grows in the summer
63.3

58.9

58.9

0.0

0.0
68. grows in the spring 0.0

69. grows in the summer

65. eaten raw

38.9

66. grows in fields

35.6

0.0

67. is a vegetable

27.8
16.7

5.6 *

2.2

12.2

58.9 *

77.8

2.2

1.1

70.0

72.2

4.4

35.6

2.?

74.4

7.8
64.4

6.7 


\section{DANDALION $(N=77)$}

\section{Essential Non-essential Not a property}

\begin{tabular}{|c|c|c|c|}
\hline 1. is various colors & 7.8 & 31.2 & 59.7 \\
\hline 2. is a herb & 16.9 & 29.9 & 53.2 \\
\hline 3. has yellow flowers & 85.7 & 9.1 & 5.2 \\
\hline 4. is red & 0.0 & 11.7 & 88.3 \\
\hline 5. has green stem & 72.7 & 19.5 & 5.2 \\
\hline 6. has fuzzy seeds & 64.9 & 15.6 & 16.9 \\
\hline 8. is a fruit & 1.3 & 18.2 & 80.5 \\
\hline 9. grows from soil & 70.1 & 14.3 & 13.0 \\
\hline 10. can be cultivated & 27.3 & 48.1 & 24.7 \\
\hline 11. is a spice & 3.9 & 17.5 & 72.7 \\
\hline 12. has green leaves & 5.3 .2 & 2.3 .4 & 22.1 \\
\hline 13. can be cooked & 28.6 & 49.4 & 22.1 \\
\hline 14. grows in gardens & 2.8 .6 & $54 \cdot 5$ & 15.6 \\
\hline 15. is a food & 24.7 & 44.2 & 31.2 \\
\hline 16. makes sauce & 3.9 & 20.8 & 72.7 \\
\hline 17. tastes good & 11.7 & 59.7 & 28.6 \\
\hline
\end{tabular}

18. used for decorating

food

19. can he dried up

20. tastes bad

21. eaten in salads

22. is a weed

23. is green

24. grows in lawns

64.9

25. has yellow petals 63.6
3. 9

11.7

3.9

24.7

67.5

16.9
36.4

59.7

40.3

46.8

57.1

37.7

44.2

29.9

20.8

10.4

31.2

49.4

31.2

3.9

16.9

16.9 
26. grows in grass

$$
80.5
$$

16.9

2.6

27. made into pie

2.6

29.9

67.5

28. is nutritious

(hea lthy)

24.7

3.9

29. is mushy

30. is many varieties

31. is smal1

31.2

32. made into wine

33. removed from lawns

$$
\text { (not liked) }
$$

51.9

34. is hard

$$
2.6
$$

74.0

35. is yellow

36. is a flower

37. has stem

38. grows in soil

$$
\text { (ground) }
$$

75.3

39. user for cooking

18.2

14.3

22.2

18.2

2.8 .6

3. 9

5.2

45. is skin-covered

46. is pretty

47. has many shapes

20.8

7.8

22. 1
42.9

39.0

26.0

45.5

61.0

49.4

18.2

16.9

$18 \cdot 2$

11.7
3.9

40.3

$79.2 *$

7.8

19.5

1.3

36.4

68.8

41.6

6.5

5

6.5

40.3

36.4

32.5

36.4

33.8

59.7

72.7

11.7

51.9

$39.0 *$

49. chilaren do not 
like it

1.3

50. is a plant

68.8

51. has lacy leaves

18.2

52. yrows in spring

71.4

53. is a wild flower 50.6

54. has white fluff

(attached to seeds)

$$
57.1
$$

55. used for seasoning 7.8

56. is edihle

50.6

57. blown away

(for good luck)

26.0

5.2

19.5

14.3

60. contains acid

61. has little or

$$
\text { no taste }
$$

2.6

0.0

22.2

63. has minerals

64. grows in the

$$
\text { summer }
$$

65. eaten raw

19.5

53.2

9.1

66. grows in fields

46.8
55.8

42.9

18.2

11.7

27.3

53.2

2.6 .0

1.3

24.7

2.2. 1

24.7

18. 2

39.0

51.9

26.0

20.8

53.2

19.5

36.4

57. 1

58.4

20.8

36.4

44.2

48.1

46.8

7. 8

92.2

42.9

35. 1

45.5

6.5

45.5

35. 1

40.3

5.2

22.1

64.9 
CATEGORY 2

FRUIT $(\mathrm{N}=166)$

Essential Non-essential Not a property

1. has many colors

53.0

42.8

42.8

$75 \cdot 3$

4. is edible

5. has sour skin

6. makes salads

7. is nutritious

$$
\text { (healthy) }
$$

8. tastes good

9. stops thirst.

10. is pear-shaped

11. is sticky

12. is a plant

13. many varieties

14. is a kind of berry

15. is green

16. can be cooked

17. used for pies

18. is mushy

19. is red inside

20. has black and white

21. has shiny skin

6.6

86.1

5.4

22. is a vegetable

8.4

24.7

65.1 


\begin{tabular}{|c|c|c|c|}
\hline 23. contains water & 42.2 & 51.8 & $4.8 *$ \\
\hline 24. is purple & 2.4 & 85.5 & 10.2 \\
\hline 5. has green rind & 4.8 & 86.1 & 7.8 \\
\hline 26. has a pit & 15.1 & 78.9 & 4.8 \\
\hline 27. is heavy & 3.0 & 77.7 & 17.5 \\
\hline 28. is oval & 4.2 & $85 \cdot 5$ & 7.8 \\
\hline ?9. is yellow & 5.4 & $86 \cdot 1$ & 7.2 \\
\hline 30. has green skin & 5.4 & 84.9 & 8.4 \\
\hline 31. grows in vines & 12.7 & 79.5 & 6.0 \\
\hline 32. is sweet & 15.7 & 80.7 & 1.2 \\
\hline 33. makes jam & 15.1 & 79.5 & $3 \cdot 6$ \\
\hline 34. is juicy & 25.9 & 68.7 & 3.6 \\
\hline 35. tastes bad & 1.8 & 74.7 & $21 \cdot 1$ \\
\hline 36. grows on bushes & $15 \cdot 1$ & 75.9 & 7.2 \\
\hline 37. is sour & 3.6 & 90.4 & 4.8 \\
\hline 38. has white rind & 4.2 & 80.7 & 13.9 \\
\hline 39. is small & 6.0 & 88.6 & 4.2 \\
\hline 40. is red & 6.6 & 84.3 & 7.2 \\
\hline 41. has qray skin & 4.2 & 69.9 & 2.3 .5 \\
\hline 42. is round (almost) & 12.0 & 82.5 & 3.0 \\
\hline 43. has seeds & 40.4 & 57.8 & 0.6 \\
\hline 44. grows in summer & $32 \cdot 5$ & 62.0 & 2.4 \\
\hline 45. is food & 78.9 & $18 \cdot 1$ & 0.6 \\
\hline 46. makes desserts & 25.3 & 69.3 & 3.6 \\
\hline 47. is expensive & 6.6 & 81.3 & 9.0 \\
\hline 48. has vitamins & $64 \cdot 5$ & $32 \cdot 5$ & $1.2 *$ \\
\hline - is soft inside & 14.5 & 81.3 & 2.4 \\
\hline
\end{tabular}


50. is various sizes 55.4

51. has rind

13.3

52. has hard skin

53. is pinkish-red

54. is large (big)

55. grows plants

from pit

56. is various shapes 58.4

6.0

5.4

3.6
42.2

1. 2

80.7

4.8

86.7

4.8

86.7

6.0

86.7

7.2

69.9

3.6

37.3

0.6 
page 115

\section{PLUM ( N=92)}

Fssential non-essential Not a property

$\begin{array}{lccr}\text { 1. has many colors } & 2.3 .9 & 43.5 & 32.6 \\ \text { 2. has skin } & 83.7 & 16.3 & 0.0 \\ \text { 3. grows on trees } & 89.1 & 9.8 & 1.1 \\ \text { 4. is edible } & 87.0 & 13.0 & 0.0 \\ \text { 5. has sour skin } & 41.3 & 47.8 & 10.9 \\ \text { 6. makes salads } & 8.7 & 60.9 & 30.4\end{array}$

7. is nutritious

(healthy)

72.8

27.2

0.0

8. tastes good

26.1

71.7

2.2

9. stops thirst

12.0

75.0

13.0

10. is pear-shaped

3.3

2.3 .9

$72.8 *$

11. is sticky

15.2

69.6

15.2

12. is a plant

38.0

21.7

40.?

13. many varieties

44.6

46.7

8.7

14. is a kind of berry

15. is green

16. can be cooked

17. used for pies

18. is mushy

19. is red insire

20. has black and

white seeds

21. has shiny skin

22. is a vegetable

$$
10.9
$$

2.2

32.6

15.2

37.0

$28 \cdot 3$

2. 2

32.6

2.2
34.8

38.0

50.9

77.2

56.5

51.1

$53.3 *$

59.8

$4 \cdot 3$

7.6

6.5

20.7

22.8

75.0

59.8

6.5

14.1

82.6 


\begin{tabular}{|c|c|c|c|}
\hline 2.3. contains water & 54.3 & 33.0 & 7.6 \\
\hline 24. is pucple & 55.4 & 44.6 & 0.0 \\
\hline 25. has qreen rind & 4.3 & 21.7 & 73.9 \\
\hline 25. has a pit. & 91.5 & 17.4 & 1.1 \\
\hline 27. is heavy & 1.1 & 37.0 & 62.0 \\
\hline 28. is oval & 5.3 .3 & 39.1 & 7.6 \\
\hline 29. is yellow & 3.3 & 37.0 & 59.8 \\
\hline 30. has green skin & 4.3 & 29.3 & 66.3 \\
\hline 31. grows in vines & 1. 1 & 28.3 & 69.6 \\
\hline 32. is sweet. & 32.6 & 60.9 & 6.6 \\
\hline 33. makes jam & 21.7 & 67.4 & 10.9 \\
\hline 34. is juicy & 52.2 & 46.7 & 0.0 \\
\hline 35. tastes bad & 2.2 & 52.2 & 45.7 \\
\hline 36. grows on hushes & 4.3 & 20.7 & 73.9 \\
\hline 37. is sour & 10.9 & 64.1 & 25.0 \\
\hline 38. has white rind & 1.1 & 23.9 & 75.0 \\
\hline 39. is small & 34.8 & 65.2 & 0.0 \\
\hline 40. is red & 33.7 & 41.3 & 25.0 \\
\hline 41. has gray skin & 4.3 & 23.3 & 68.5 \\
\hline 42. is round (almost) & 50.0 & 41.3 & 8.7 \\
\hline 43. has seeds & 41.3 & 2.5 .0 & 33.7 \\
\hline 44. grows in summer & 39.1 & 55.4 & 4.3 \\
\hline 45. is food & 88.0 & 10.9 & 1.1 \\
\hline 46. makes desserts & 19.6 & 80.4 & 0.0 \\
\hline 47. is expensive & 6.5 & 68.5 & 23.9 \\
\hline 48. has vitamins & 68.2 & 33.7 & 1. $1 *$ \\
\hline 49. is soft inside & 58.7 & 39.1 & 0.0 \\
\hline
\end{tabular}


50. is various sizes 50.5

51. has rind

52. has hard skin

5.3. is pinkish-red

54. is large (big)

55. grows plants

from pit

56. is various shapes

57. is a fruit

8.7

28.3

5.4

47.8

27.2

94.7
42.4

1. 1

23.9

68.5

23.9

67.4

48.9

2.8

65.2

29.3

39.1

12.0
45.7

27.2

5.4

0.0 
page 118

WATERMELON ( $N=75)$

Essential Non-essential Not a property

1. has many colors

13.3

28.0

58.7

2. has skin

68.0

2.2 .7

9.3

3. grows on trees

0.0

10.7

89.3

4. is edible

84.0

1.3 .3

2.7

5. has sour skin

18.7

42.7

37.3

6. makes salads

18.7

54.7

26.7

7. is nutritious

(healthy)

8. tastes good

9. stops thirst

10. is pear-shaped

11. is sticky

12. is a plant

13. many varieties

14. is a kind of berry

15. is green

16. can be cooked

17. used for pies

18. is mushy

19. is red inside

20. has black and whje
21. has shiny skin

22. is a vegetable

23. contains water
68.0

53.3

40.0

28.0

1.3

22.7

58.7

25.3

4. 0

49.3

6.7

5.3

14.7

76.0

28.0

9.3

81.3
41.3

5.3

54.7

2.7

57.3

13.3

24.0

5.3 .3

74.7

22.7

21.3 *

44.0

76.0

17.3

40.0

10.7

44.0

48.0

48.0

46.7

57.3

2.5 .3

18.7

4. 0

25.3

6.7

49.3

21.3

14.7

76.0

17.3

1. 3 


\begin{tabular}{|c|c|c|c|}
\hline 24. is purple & 2.7 & 14.7 & 82.7 \\
\hline 25. has green rind & 69.3 & 18.7 & 12.0 \\
\hline 26. has a pit & 10.7 & 17.3 & 69.3 \\
\hline 27. is heavy & 36.0 & 60.0 & 1.3 \\
\hline 28. is oval & 73.3 & 22.7 & 0.0 \\
\hline 29. is yellow & 0.0 & 20.0 & 78.7 \\
\hline 30. has green skin & 69.3 & 22.7 & 8. 0 \\
\hline 31. qrows on yines & 56.0 & 18.7 & 22.7 \\
\hline 32. is sheet & 49.3 & 49.3 & 1.3 \\
\hline 33. makes jam & 4.0 & 49.3 & 44.0 \\
\hline 34. is juicy & 65.3 & 26.7 & 3.0 \\
\hline 35. tastes bad & 1.3 & 49.3 & 48.0 \\
\hline 36. grows on bushes & 5.3 & 20.0 & 73.3 \\
\hline 37. is sour & 0.0 & 30.7 & 68.0 \\
\hline 38. has white cind & 58.7 & 20.0 & 18.7 \\
\hline 39. is small & 1.3 & 62.7 & 34.7 \\
\hline 40. is red & 48.0 & 44.0 & 6.7 \\
\hline 41. has qray skin & 0.0 & 14.7 & 85.3 \\
\hline 42. is round (almost) & 29.3 & 49.3 & 18.7 \\
\hline 43. has sferts & 90.7 & 9.3 & 0.0 \\
\hline 44. grows in summer & 69.3 & 24.0 & 2.7 \\
\hline 45. is food & 90.7 & 8.0 & 0.0 \\
\hline 46. makes dessets & 21.3 & 65.3 & 13.3 \\
\hline 47. is expensive & 2.7 & 78.7 & 17.3 \\
\hline 48. has vitamins & 70.7 & 28.0 & $1.3 *$ \\
\hline 49. is soft inside & 40.0 & 56.0 & 4.0 \\
\hline 50. is vacious sizes & 52.0 & 44.0 & 4.0 \\
\hline
\end{tabular}


51. has rind

81.3

52. has hard skin

58.7

85.3

37.3

55. grows plants

from pit 28.0

56 . is vacious shapes

42.7

31.3

57. is a fruit
12.0

36.0

10.7

58.7

21.3

44.0

10.7
4.0

5.3

4. 0

4.0

46.7

12.0

5.3

1.3 
page 121

GOOSRBFERY $(N=90)$

Fssential Non-essential Not a property
1. has many colors
6.7
42.2
$38.9 *$
2. has skin
47.8
35.6
5.6
3. grows on trees
13.3
27.8
46.7
4. is edible
22.2
24.4
2.2
5. has sour skin
6.7
63.3
16.7
6. makes salads
3.3
42.2
42.2

7. is nutritious

(healthy)

8. tastes good

9. stops thirst.

10. is pear-shaped

11. is sticky

12. is a plant

13. inany varieties

14. is a kind of berry 81.1

15. is green

16. can be cooked

17. uspa for pies

18. is mushy

19. is red inside

20. has black and white
7.8

38.9

$3 \cdot 3$

22. is a vegetable

23. contains water

21. has shiny skin

41.1
44.4

10.0

4.4

4.4

15.6

43.3

17.8

4.4

34.4

15.6

$14 \cdot 4$

30.0
43.3

1.1

70.0

8.9

56.7

26.7

28.9

54.4

60.0

13.3

2.4 .4

20.0

45.6

23.3

7.8

0.0

37.8

4.5. $6 *$

52.2

2.2

63.3

5.6

58.9

14.4

44.4

13.3
37.8

42.2

21.1

42.2
41. 1

8.9

63.3

6.7 


\begin{tabular}{|c|c|c|c|}
\hline 24. is purple & 16.7 & 45.6 & $21.1 *$ \\
\hline 25. has green rind & 7.8 & 18.9 & 64.4 \\
\hline 26. has a pit & 15.6 & $24 \cdot 4$ & 47.8 \\
\hline 27. is heavy & 1.1 & 38.9 & 51.1 \\
\hline 28. j.s oval & 12.2 & $52 \cdot 2$ & 23.3 \\
\hline 29. is yellow & 11.1 & 40.0 & 38.9 \\
\hline 30. has green skin & 8.9 & 37.8 & 40.0 \\
\hline 31. grows in vines & 43.3 & 22.2 & $24.4 *$ \\
\hline 32. is sweet & 17.8 & 61.1 & 8.9 \\
\hline 33. makes jam & 35.6 & 53.3 & 1.1 \\
\hline 34. is juicy & 26.7 & 55.6 & 3.3 \\
\hline 35. tastes bad & 5.6 & 70.0 & 15.6 \\
\hline 36. grows on bushes & 48.9 & 31.1 & 7.8 \\
\hline 37. is sour & $14 \cdot 4$ & 65.6 & 7.8 \\
\hline 38. has white rind & 2.2 & 26.7 & 58.9 \\
\hline 30. is small & 60.0 & 27.8 & 1.1 \\
\hline 40. is red & 18.9 & 47.8 & $21 \cdot 1$ \\
\hline 41. has gray skin & 7.8 & 28.9 & 54.4 \\
\hline 42. is round (almost) & 66.7 & 21.1 & 1.1 \\
\hline 43. has seeds & 42.2 & 36.7 & 7.8 \\
\hline 44. grows in summer & 37.8 & 51.1 & 11.1 \\
\hline 45. is food & 67.8 & 20.0 & 0.0 \\
\hline 46. makes desserts & 30.0 & 57.8 & 1.1 \\
\hline 47. is expensive & 0.0 & 54.4 & 32.2 \\
\hline 48. has vitamins & $41 \cdot 1$ & 40.0 & $5.6 *$ \\
\hline 49. is soft inside & $32 \cdot 2$ & 53.3 & 2.2 \\
\hline 50. is various sizes & 26.7 & 44.4 & 17.8 \\
\hline
\end{tabular}


51. has rind

52. has hard skin

10.0

21.1

6.7

11.1

3.2

54. is large (hig)

17.8

Erom pit

56. is various shapes

14.4

57. is a fiuit

75.6
34.4

48.9

35.6

32.2

42.2

11.1
57.8

$47.8 *$

26.7

51.1

38.9

31. 1

2.2 


$$
\text { AVOCADO (N=75) }
$$

Essential Non-essential Not a property

1. has many colors

2. has skin

3. grows on trees

4. is edible

5. has sour skin

6. makes salads

7. is nutritious

$$
\text { (healthy) }
$$

8. tastes good

9. stops thirst

10. is pear-shaped

11. is sticky

12. is a plant

13. many varieties

14. is a kind of berry

15. is green

16. can be cooked

17. used for pies

18. is mushy

19. is red inside

20. has black and white seeds

21. has shiny skin

22. is a vegetable

23. contains water

$$
6.7
$$

$$
70.7
$$

$$
45.2
$$

88.0

2. 7

17.3

5.3 .3

14.7

5.3

29.3

6.7

76.0

24.0

4.0

$65 \cdot 3$

30.7

2.7

22.7

4.0

4. 0

40.0

48.0

36.0
33.3

2.4 .0

22.7

8.0

65.3

56.0

56.0

1. 3

$26.7 *$

1.3

26.7

22.7

41.3

1. 3 *

47.3

5.3

38.7

49.3

29.3

14.7

58.7

30.7

18.7

2.7

26.7

41.3

24.0

62.7

28.0

2.7

49.3

16.0

44.0

49.3

58.7

13.3

24.0

66.7 
24. is purple

1.3

24.0

30.7

18.7

58.7

45.3

30.7

28.0

25.3

53.3

30.7

48.0

62.7

32.0

58.7

30.7

64.0

22.7

25.3

42.7

24.0

57.3

8.0

69.3

65.3

30.7

46.7

$49 \cdot 3$
69.3

34.7

6.7

28.0

30.7

61.3

6.7

65.3

24.0

58.7

26.7

37.0

44.0

33.3

49.3

14.7

70.7

68.0

22.7

37.3

10.7

6.7

24.0

22.7

2.7

2.7

22.7 
51. has rind

$$
24.0
$$

52. has hard skin 22.7

53. is pinkish-rod

0.0

54. is large (biq)

5.0

55. grows plants from pit

$$
70.7
$$

56. is various shapes 20.0

57. is a fruit.
28.0

44.0

28.0

50.7

44.0

29.3

65.3

41.3
18.7

48.0

14.7
6.7

26.7

48.0

30.7 
CATEGORY 3

$$
\text { BIRD ( N=167) }
$$

Fissential Non-essential Not a property

1. is a house pet.

(domesticated)

2. is ugly

3. moves fast

4. can talk

5. catch fish

6. has short wings

7. eats seeds

8. is coloctul

9. Lives in cage

10. symbol of death

11. is black

12. Found in zoo

13. Lays eggs

14. is wild (untamed)

15. chicps

16. Flies

17. has short legs

18. Iives in cold weather

(winter,snow)

7.2

19. perches in trees

(branches)

34.1

3.6

81.4

10.8

20. is large (big)

21. is bad

$$
78.4
$$$$
5.4
$$

0.0

77.8

21.6

71.9

3.6

76.0

18.6

84.4

6.0

13.8

83.8

1. 2

37.1

61.1

0.6

18.0

80.8

0.6

78.4

6.0

49.1

48.5

89.8

6.0

87.4

2.4

26.9

0.6

81.4

1.8

44.3

0.6

27.5

0.0

77.8

0.6 

(evil, mean)
1.8
58.1
38.3

22. migrates (filies

south in winter) 28.1

69.5

1.2

23. sings

31.1

65.3

3.0

24. Iives in trees

39.5

59.3

0.6

25. is multicolored 14.4

82.6

1.6

26. has feathers

81.4

18.0

0.6

27. Lives in desert

3.0

83.2

13.2

28. is domestic (tamed) 7.8

83.2

7.8

29. is small (1ittle) 7.8

89.2

1.8

30. circles around

prey (victim)

7.8

31. eats worms

32.9

32. has beak (bill)

86.2

33. Lives in tropical

areas

8.4

34. has webbed feet.

18.6

35. is powerful

3.6

36. wardiles

$$
\text { (walks Eunny) }
$$

6.6

37. can be trained

21.0

38. has claws

32.9

39. Found in Alaska

4. 2

40. is amusing

(entertaining)

$4 \cdot 6$

77.2

10.2

41. many species (types) 


\section{$\operatorname{mag} 1200$}



animals

$$
9.0
$$

81.4

7.8

43. has large beak

8.4

86.2

4. 2

44. cannot fly

6.0

52.7

$39.5 *$

45. has (two) wings

79.0

18.6

1.2

46. 1ives in nests

44.9

5.3 .3

0.5

47. is brown

3. 0

88.0

7.2

43. preys on dead (or a lmost

$$
\text { read) flesh }
$$

6.6

82.6

10.2

49. has (two) eyes

79.0

19.8

0.6

50. is black and white 4.2

87.4

7.3

51. is free

19.8

74.3

5.4

52. Iives in North

$$
\text { pole }
$$

$$
3.6
$$

74.3

5.4

53. resembles tuxedo 2.4

67.1

$29.3 *$

54. has (two) feet

69.5

26.3

1. 2

55. swims

10.2

67.7

$16.2 *$

56. is an animal

73.1

13.8

$12.0 *$

57. can mimick (imitate) 


$$
\text { page 130 }
$$

$$
\text { SPARRNA }(N=72)
$$

Fisential Non-essential Not a property

1. is a house pet.

(aomesticated)

2. is ugly

3. moves fast

4. $\operatorname{can} \operatorname{talk}$

5. catch fish

6. has short wings

7. eats seeds

8. is colorful

9. Iives in cage

10. symhol of death

11. is black

12. found in zoo

13. lays eqgs

14. is wild (untamed)

15. chirps

16. flies

17. has short legs

18. Lives in cold weather
34.7

59.7

54.2

26.4

26.4

22.2

43. 1

44.4

62.5

2.8

47.2

59.7

19.4

44.4

16.7

4.2

48.6
$59.7 *$

36.1

1. 4

66.7 *

56.9

1. 4

6.9

41.7

30.6

19.4

40.3

34.7

1.4

9.7

0.0

0.0

47.2

2.8 
22. migrates (flies

south in winter) 45.8

23. sings

24. lives in trees

25. is muticolored

26. has feathers

27. lives in desert

2.8

28. is domestic (tamed) 5.6

29. is sinall (little) 76.4

30. circles around

prey (victim)

5.6

31. eats worms

61.1

32. has beak (bill)

76.4

33. lives in tropical areas

$$
2.8
$$

34. has webbed feet.

19.4

35. is powerful

4.2

36. waddles

$$
\text { (walkes funny) }
$$

$4 \cdot 2$

9.7

47.2

2.8

4.3 .1

19.4

45.8

$58 \cdot 3 *$

4.2

5.6

13.9

51.4

56.9

47. 2*

37. can he trained

38. has claws

39. found in Alaska

40. is amusing

(entertaining)

$4 \cdot 2$

41. many species

$$
\text { (types) }
$$

47.2

42. preys on small 


$$
\text { anima } 15
$$

43. has large beak

44. cannot fly

5.7

20.8

4.2

45. has (two) wings

81.9

45. 1ives in nests

69.4

47. is brown

41.7

48. preys on dead for almost

$$
\text { dead) flesh }
$$$$
4.2
$$

26.4

66.7

49. has (two) eyes 86.1

50. is hlack and white 8.3

51. is free 36.1

52. Lives in North

$$
\text { Pole }
$$$$
5.6
$$

53. resembles tuxedo

$$
4.2
$$

79.2

54. has (two) feet

$$
5.6
$$

8. 3

41.7

48.6

$$
1.4
$$

44.4

11.1

55. swims

75.0

56. is an animal

57. can mimick (imitate)

$$
8.3
$$

37.5

22.2

22.2

6.9
51.4

47.2

84.7

5.6

1. 4

13.9
58. has (two) legs 76.4

19.4

70.8

15.3

77.8

12.5

6.9

29.2

62.5 *

53. Iives in Antartica 4.2

91.7

9.7

12.5

60. is a bird

50.0

69.4

69.4

1. 4

91.7

$6 \cdot 9$ 


\author{
PARROT ( $N=83)$ \\ Fissential Non-essential not a property
}

1. is a house pet

(domesticated)

41.0

57.8

1.2

2. is ugly

2.2

60.2

36.1

3. moves fast

14.5

65.1

20.5

4. can talk

59.0

39.8

1.2

5. catch fish

4.8

43.4

$51.8 *$

6. has short wings

$24 \cdot 1$

56.6

$18.1 *$

7. eats seeds

43.4

54.2

2.4

8. is colorful

55.4

43.4

1. 2

9. Iives in cage

26.5

72.3

1.2

10. symbol of death

3.6

16.7

79.5

11. is black

4. 8

54.2

39.8

12. Found in zoo

28.9

68.7

$2.4 *$

13. 1ays eggs

51.8

37.3

10.8

14. is wild (untamed) 10.8

74.7

14.5

15. chirps

43.3

39.8

15.7

16. flies

76.9

18.1

6.0

17. has short legs

44.6

55.4

0.0

18. lives in cold weather
(winter, snow)
7.2

21.7

71.1

19. perches in trees

(branches)

20. is large (big)

21. is had

$$
36.1
$$

9.6

2.4
60.2

3.6

56.6

33.7

(evil, mean)

47.0

50.6 
22. migrates (flies

south in winter

$$
8.4
$$

$45.8 *$

45.8 *

23. sings

33.7

59.0

6.0

24. lives in trees

45.8

51.8

2.4

25. is multicolored

57.8

39.8

2.4

26. has feathers

88.0

12.0

0.0

27. Lives in desert

2.4

34.9

62.7

28. is domestic

38.6

57.8

3.6

29. is small (little) 32.5

6.3 .9

3.6

30. circles around

prey (victim)

0.0

41.0

57.8

31. eats worms

19.3

65.1

15.7

32. has beak (bill)

96.4

2.4

0.0

33. lives in tropical

$$
\text { areas }
$$

55.4

43.4

1.2

34. has webbed feet.

9.6

31.3

56.6

35. is powerful

3.6

47.0

49.4

36. wadiles

$$
\text { (walks funny) }
$$

15.7

41.0

$37.3 *$

37.

51.8

44.6

3.6

38. has claws

59.0

32.5 .

8.4

39. found in Alaska

1.2

22.9

74.7

40. is amusing

$$
\text { (entertaining) }
$$

60.2

$$
6.0
$$

41. many species

$$
\text { (types) }
$$

41.0

47.0

12.0

42. preys on small 
animals

43. has large beak

$$
12.0
$$

47.0

0.0

25.3

7.2

66.3

60.2

4.8

47. is brown

19.3

46. 1 ives in nests

48. preys on dead (or almost

dead) flesh 4.8

42.2

$14 \cdot 5$

51.8

62.7

21.7

52. lives in North

pole

0.0

15.7

20.5

13.3

25.3

6.0

78.3

57. can mimick (imitate)

$$
65.1
$$

58. has (two) legs

79.5

59. Lives in Antartic

0.0

60. is a bigd

92.8

61. has many species

0.0

62. Iives in cage
33.7

19.3

2.0 .5

6.0

0.0

71.1
41.0 *

0.0

59.0

1. 2

13.3

34.9

51.8

2.4

44.6

13.3

$84.3 *$

77.1

2.4

68.7

15.7

1. 2

1. 2

$78.3 *$

1.2

0.0

3.6 


\section{VULTURS $(\mathrm{N}=72)$}

Essential Non-essential Not a property

1. is a house pet

(domesticated)

2.8

30.6

51.4

1.4

4. can talk

5. catch fish

6. has short wings

7. eats seeds

8. is colorful

9. Iives in cage

10. symbol of death

11. is black

12. found in zoo

13. Iays eqgs

14. is wild (untamed)

15. chirps

16. flies

17. has short legs

$$
\text { (winter, snow) }
$$

19. perches in trees

$$
\text { (branches) }
$$

20. is large (big)

21. is bad

$$
\text { (evil, mean) }
$$

36.2

4.2

48.6

70.8
13.9

56.9

41.7

16.7

38.9

34.7

43.1

41.7

45.8

37.5

62.5

68.1

29.2

25.0

33.3

9.7

52.8

37.5

2.7 .8

50.0

13.9
81.9

12.5

4. 2

80.6

45.8

61. 1

50.0

58.3

52.8

6.9

8.3

23.6

2.9

0.0

61.1

1.4

26.4

55.6

2.2. migrates (flies 
south in winter)

$$
2.8
$$

34.7

59.7

23. sings

1.4

37.5

61.1

24. Lives in trees

31.9

$58 \cdot 3$

9.7

25. is multicolored

4.2

50.0

44.4

26. has feathers

90.3

5.6

4. 2

27. 1ives in desert

41.7

36.1

20.8

28. is domestic (tamed)

$$
2.8
$$

18.1

$75.0 *$

29. is small (little) 1.4

40.3

56.9

30. circles around

prey (victim) $\quad 34.7$

13.9

0.0

31. eats worms

20.8

43.1

34.7

32. has heak (bill)

72.2

20.8

5.6

33. lives in tropical

areas

34. has webbed feet

35. is powerful

36. wadiles

$$
\text { (walks funny) }
$$

6.9

37. can he trained

9.7

38. has claws

93.1

4.2

39. found in Alaska

40. is anusing

$$
\text { (entertaining) }
$$

1.4

41. many species

$$
\text { (types) }
$$

$43 \cdot 1$

42. preys on small
30.6

66.7

47.2

38.9

20.8

68.1

18.1

1.4

34.7

55.6

43.1

47.2

6.9

0.0 *

45.8

47.2 15.3 

animals
0.0
0.0
0.0

43. has large heak

63.9

30.6

4. 2

44. cannot $f 1 Y$

1.4

9.3

90.3

45. has (t.wo) wings

91.7

6.9

1.4

46. lives in nests

62.5

30.6

6.9

47. is hrown

12.5

63.9

20.8

48. preys on dead (or almost

$$
\text { dead) flesh }
$$

49. has (two) eyes

83.3

50. has beak (bil1)

22.2

51. is free

52.8

52. 1 ives in North

Pole

$$
0.0
$$

62.5

56. is an animal

57. can mimick

$$
\text { (imitate) }
$$

0.0

58. has (two) leqs

79.2

59. Iives in Antartica

$4 \cdot 2$

60. is a hird

97.2

61. has many species
25.0

11.1

12.5

27.8

19.4

4.2

0.0

15.3

1.4

50.0

26.4

5.6

40.3

70.8

84.7

1.4

66.7

18.1

19.4

80.6

16.7

4.2

22.2

70.8

1.4

1.4

50.0

12.5 
page 139

PFNGIIIN ( N=83)

Fssential Non-essential Not a property

1. is a house pet

(lomesticated)

25.7

73.5

1.2

2. is ugly

2.4

65.1

$32.5 *$

3. moves fast

4.8

57.8

37.3

4. can talk

1.2

21.7

$77.1 *$

5. catch fish

65.1

27.7

7. 2

6. has short wings

54.2

34.9

9.6

7. eats seeds

4.8

49.4

44.6

8. is colorful

14.5

41.0

44.6

9. Iives in caqe

1.2

56.6

42.2

10. symbol of death

2.4

21.7

73.5

11. is black

32.5

49.4

16.9

12. found in 200

12.0

78.3

7.2

13. lays eggs

41.0

45.8

13.3

14. is wild (untamed)

33.7

56.6

8.4

15. chirps

16.9

30.1

51.8

16. flies

9.6

15.7

$74.7 *$

17. has short legs

74.7

25.3

0.0

18. Iives in cold weather

$$
\text { (winter, snow) } \quad 90.4
$$

5.4

0.0

19. perches in trees

(hranches)

20. is large (big)

21. is bad

(evil, mean)
2.4

10.8

1.2
12.0

81.9

68.7

20.5 
22. miqrates (filies

south in winter)

$$
2.4
$$

20.5

77.1

23. sings

0.0

33.7

66.3

24. lives in trees

1.2

10.8

88.0

25. is multicolored

19.3

43.4

37.3

26. has feathers

36.1

24.1

38.6

27. lives in desert.

10.8

86.7

28. is domestic (tamed) 4.8

59.0

36.1

29. is small (little) 34.9

51.8

13.3

30. circles around

prey (victim)

3.6

31. eats worms

4.8

32. has beak (bill)

33. Iives in tropical areas

3.4

34. has webbed feet

77.1

7.2

35. is powerful.

36. waddles

$$
\text { (walks funny) }
$$$$
77.1
$$

37. can he trained

19.3

32.5

56.6

39. found in Alaska

40. is amusing

(is entertaining) 22.9

41. many species

$$
\text { (types) }
$$$$
27.7
$$

42. preys on small
19.3

2.4

$4 \cdot 8$

86.7

15.7

6.0

62.7

30.1

68.7

12.0

30.1

37.3

32.5

10.8

69.6

7.2

25.3 

animals
0.0
0.0
0.0

43. has large beak

45.8

42.2

12.0

44. cannot fly

67.5

22.9

2. 6

45. has (two) wings

71.1

20.5

4.8

46. Iives in nests

9.6

31.3

59.0

47. is brown

$2 \cdot 4$

2.8 .9

68.7

48. preys on dead for almost

dead) flesh 12.0

41.0

45.8

49. has (two) eyes

79.5

19.3

1. 2 *

50. is black and white 88.0

10.8

0.0

51. is free

27.7

60.2

12.0

52. Iives in North

Pole

53. resembles tuxero 54.2

54. has (two) feet 88.0

55. Swims

56. is an animal

57. can minick

(imitate)

3.6

53. has (two) $\operatorname{legs}$

83.1

59. 1ives in Antartica 60.2

60. is a bird

85.5

61. has many species 24.1

$$
36.1
$$

7.2

44.6

1. 2

9.6

2.4

18.1

3.6

4.8

8.4

21.7

73.5

1.3. 3

2.4

33.7

$6.0 *$

8.4

6.0

47.0

27.7 
page 742

CATEGORY 4

FURNITURE ( N=167)

Essential Non-essential Not a property

1. made of plastic 7.8

86.8

4.8

2. found in kitchen

28.1

69.5

1.8

3. used for sitting on

(or ąt)

4. is comfortable

50.3

21.6

5. has flat surface

18.6

6. is a container

4. 8

7. holds objects

20.4

8. has tall legs

3.6

9. is short (low)

5.4

10. a vaciety of sizes 58.1

11. is expensive

10.2

12. is a type of seat

$$
\text { (small chair) }
$$

13. is gray

14. many types

15. is hard

16. has no back

17. holds (old)

18. used for writing

19. found in bars

20. made of glass

21. can be decorated

22. is cylindrical
19.2

0.6

66.5

11.4

4.8

4. 8

9.6

18.0

10.2

17.4

7. 2
47.9

76.6

77.8

76.6

70.1

90.4

90.4

40.1

82.6

$0.6 *$

1. 2

2.4

34. 1

9.0

5.4

3.6

1.2

6.0

3. 6

76.0

12.0

85.6

1. 2 .

83.2

3.0

83.8

10.8 *

71.9

22.2

70.1

18.6

78.4

3. 0

83.2

5.4

79.0

3.0

77.2

14.4 
2.3. holds objects

18.6

73.1

6.0

24. can be uncomfortable

$$
16.2
$$

81.4

1. 2

25. surrounded by chairs

7.8

26. is round

9.6

27. found in most huildings

$$
56.3
$$

28. holds waste

$$
\text { (garbage) }
$$

$$
\text { 7. } 2
$$

29. is various colors 42.5

30. used for decoration

$$
30.5
$$

31. found near (under)

desks

$$
9.0
$$

32. made of wood

24.6

33. has smooth

$$
\text { (finish) top }
$$

15.0

34. put things in

$9 \cdot 0$

35. emptied when full 1.8

36. made of cushions 14.4

37. is smelly (dirty) 1.2

38. found against

$$
\text { (close to) a wall } 4.2
$$

39. is square

10.2

40. is supportive

$$
\text { (put things on) }
$$

79.0

11.4

85.0

6.6

85.6

3.6

38.3

2.4

45.5

$45.5 *$

55.7

0.6

67.1

1.2

73.1

0.6

79.6

4.2

74.9

15.6

44.3

52. 1

78.4

6.0

45.5

57.5

88.0

7.2

83.8

5.4

62.9

3.6 
41. used as a step

(stand on)

7.8

76.0

15.0

42. found in libraries

$$
26.9
$$

69.5

1.8

43. is dack colored

5.4

44. is tall (high)

6.0

45. d variety of styles

40.1

0.6

46. made of metal

7.8

87.4

4.2

47. is oval

7.2

85.6

6.0

48. has shelves

9.0

85.6

$4 \cdot 8$

49. used for studying 11.4

82.6

5.4

50. Found in study room

$$
23.4
$$

72.5

1.8

51. has a round top

11.4

83.5

3.0

52. is functional

(useful)

67.7

29.9

3.0

53. made of (covered

$$
\text { with) cloth }
$$

10.2

82.6

4.8

54. usfd in homes

$55 \cdot 1$

40.7

1.?

55. has $(1-4)$ leqs

60.5

$37 \cdot 1$

1.8

56. Found in rooms

60.5

37.1

$1 . B$

57. is small (narrow) 3.0

91.0

5.4

58. used as a footrest 9.0

86.8

5.4

59. is rectangular

6.0

89.2

4.2

60. holds trinkets fornaments. candles)

7. 2

81.4

10.8 
61. is a variety of shapes

$$
58.7
$$

40.7

. 0.6

62. used for working

15.6

73.1

9.6 
TABLE $(N=91)$

Essential Non-essential Not a property

1. made of plastic

2. found in kitchen

3. used for sitting
8.8

36.3

47.3

4. is comfortable

5. has flat surface

6. is a container

7. holds objects

8. has tall leos

9. is short

10. a variety of sizes

9.9
5.5

86.8

52.6

40.3

58.2

26.4

30.8

60.4

85.7

81.3

53.8

11. is expensive

12. is a type of seat

$$
\text { (small chair) }
$$

13. is gray

14. many types

15. is hard

16. has no back

17. holds (old) papers

18. used for writing

19. found in bars

20. made of glass

21. can be decorated

22. is cylindrical
24.2

13.2

19.8

$$
\text { 2. } 2
$$

1.1

54.9

62.6

39.6

3.3

24.3

11.0
45. 1

86.8

27.5

87.9

44.1

36.3

39.6

65.9

67.0

71.4

86.8

74.7

69.2
1.1

8. 8

4.4

1. 1

8.8

36.3

1. 1

62.6

12. 1

4.4

7.7

69.2

11.0

1. $1 *$

1.1

19.8

30.8

7.7

3.3

0.0

$5 \cdot 5$

18.7 
23. holds objects

$$
39.5
$$

47.3

12.1

24. can be uncomfortable

$$
8.8
$$

70.3

19.8

25. surrounded by chairs

$$
29.7
$$

68.1

2.2

26. is round

15.4

82.4

2.2

27. found in most buildings

$$
47.3
$$

50.5

2.?

28. holds wast.e

$$
\text { (garbaqe) } 3.3
$$

18.7

78.3

29 . is various colors 35.2

6.3 .7

1. 1 *

30. used for decoration

$$
14.3
$$

78.0

$$
6.6
$$

31. found near

$$
\text { (under) desk } 2.2
$$

42.9

54.9

32. made of wood

30.8

68.1

0.0

33. has smooth

$$
\text { (Einish) top } \quad 48.4
$$

49.5

2.2

34. put things in

9.9

45.1

45.1

35. emptied when full 6.6

25.3

68.1

36. made of cushions

0.0

36.3

$63.7 *$

37. is smelly (dirty) 4.4

52.7

41.8

38. Eound against

$$
\text { (close to) a wall } 4.4
$$

85.7

9.9

39. is square

15.4

83.5

1.1

40. is supportive

$$
\text { (put things on) }
$$

62.6

35.2 
41. usfi as a step
(stand on)
$3 \cdot 3$
40.3
54.9

42. found in libraries

$$
27.5
$$

43. is dark-coloreã

4.4

44. is tall (high)

$3 \cdot 3$

45. a variety of styles

$$
54.9
$$

46. made of metal

7.7

47. is oval

11.0

48. has shelves

$2 \cdot 2$

49. used for studying 23.1

50. found in study Inom

$$
20.9
$$

51. has a round top

13.2

52. is functional

67.0

53. made of (covered

$$
\text { with) cloth }
$$

8.8

54. used in homes

42.9

55. has $(1-4)$ legs

71.4

56. Eound in rooms

40.7

57. is small (narrow)

6.6

58. used as a Eootrest 2.2

59. is rectangular

11.0

60. holds trinkets

(ornaments, candles) $\begin{array}{ll}71.4 & 1.1\end{array}$

$92.3 \quad 3.3$

$\begin{array}{ll}84.6 & 7.7\end{array}$

$44.0 \quad 1.1$

$87.9 \quad 3.3$

$87.9 \quad 1.1$

$\begin{array}{ll}4.5 .1 & 52.7\end{array}$

$\begin{array}{ll}73.6 & 3.3\end{array}$

$\begin{array}{ll}76.9 & 2.2\end{array}$

$83.5 \quad 3.3$

$33.0 \quad 0.0$

$\begin{array}{ll}75.8 & 15.4\end{array}$

$56.0 \quad 1.1$

$\begin{array}{ll}27.5 & 1.1\end{array}$

$59.3 \quad 0.0$

$89.0 \quad 4.4$

$56.0 \quad 41.8$

$85.7 \quad 3.3$ 
page 149

61. is a variety of shapes

$$
48.4
$$

49.5

2.2

62. used for working 26.4

71.4

2.2

63. is a furniture

89.0

9.9

1.1 
BOOKCASE $(\mathrm{N}=76)$

Essential Non-essential Not a property

1. made of plastic

2. found in kitchen

3. used for sitting

$$
\text { on (or at) }
$$

4. is comfortable

5. has flat surface

6. is a container

7. holas objects

8. has tal1 legs

9. is short

10. a variety of sizes

$$
50.0
$$

11. is expensive

12. is a type of seat

$$
\text { (small chaic) }
$$

13. is gray

14. many types

15. is hard

16. has no back

17. holas (old) papers

72.4

0.0

2.6

50.0

4.3 .4

3.9
3.9
82.9
13.2
1. 3
2.6
50.0

1.3

31.6

65.8

0.0

34.2

65.8

55.2

42.1

2.6

50.0

25.0

23.7

27.6

0.0

3.8

67.1

28.9 *

0.0

85.5

14.5

48.7

1.3

85.5

10.5

3.9

26.3

73.7

78.9

17.1

47.4

$2 \cdot 6$

48.7

6.6

76.3

19.7
15.8

18. used for writing

19. found in bars

20. made of glass

21. can be decorated
3.9

1.3

2.3

7.9
68.4

14.5

71.1

25.0

57.9

40.8

17.1

77.6

80.3

11.8 
22. is cylindrical

$$
5.3
$$

47.4

47.4

23. holds objects

24. can be uncomfortable

$$
3.9
$$

31.6

25. surrounded by chairs

26. is round

0.0

59.2

40.8

27. found in most buildings

$$
19.7
$$

69.7

10.5

28. holds waste

(garbage)

1.3

29. is various colors 28.9

30. used for decoration

$$
15.8
$$

78.9

5.3

31. found near

$$
\text { (under) desk } 7.9
$$

14.5

33. has smooth

$$
\text { (finish) top } 25.0
$$

34. put things in

63.2

35. emptied when full 3.9

36. made of cushions 1.3

37. is smelly (Airty) 2.6

38. found against

$$
\text { (close to) a wall } 26.3
$$

39. is square $10 \cdot 5$

40. is supportive
26.3

71.1

68.4 2.6 

(put things on)
86.8
13. 2
0.0

41. used as a step
(stand on)
1.3
44.7
52.6

42. Eound in 1ibraries

$\begin{array}{lll}68.4 & 30.3 & 1.3 \\ 1.3 & 89.5 & 7.9 \\ 5.3 & 89.5 & 5.3\end{array}$

45. a variety of styles

$$
50.0
$$

46.1

3.9

46. made of metal

7.9

88.2

3.9

47. is oval

0.0

52.6

43.4

48. has shelves

39.5

9. ?

1.3

49. used for studying 15.8

52.6

41.6

50. found in study coom

$$
34.2
$$

61.8

51. has a cound top

0.0

60.5

38.2

52. is functional

(usiefu 1)

68.4

27.2

3.9

53. made of

(covered with) cloth

$$
2.6
$$

59.2

38.2

54. user in homes

27.6

68.4

3.9

55. has $(1-4)$ legs

23.7

57.9

18.4

56. found in rooms

39.5

53.9

5.3

57. is small. (narcow) 5.3

80.3

14.5

58. used as a footrest 1.3

36.8

61.8

59. is rectangular

14.5

81.6

3.9 
60. holds trinkets

(ornaments, candles)
10.5
69.7
19.7

61. is a variety of shapes

42.1

62. used for working 14.5

63. is a furniture 84.2
56.6

1.3

53.9

30.3

15. 8

30.3 
STOOL $(N=91)$

Fssential Non-essential Not a property

1. made of plastic

6.6

6.6

2. found in kitchen

3. used for sitting

$$
\text { on }(o r a t)
$$

4. is comfortable

5. has flat surface

6. is a container

7. holns objects

8. has tall legs

9. is short

10. a variety of sizes

65.9

9.9

64.0

1. 1

5.5

7.7

16.5
80.2

87.9

12.1

5.5

28.6

5.5

$$
37.4
$$

11. is expensive

1. 1

12. is a type of seat

$$
\text { (sma11 chair) }
$$

67.0

2.2

13. is gray

14. many types

46.2

15. is hard

44.0

16. has no back

74.7

17. holds (old) papers

1. 1

19. used for writing

0.0

19. found in bars

26.4

20. made of glass

2.2

21. can be decorated

12.1

22. is cylindrical

78.0 20.9 8.8 1. 1 80.2 38.5 9.9

1. 1

82.4 
23. holds objects

24. can be uncomfortable

$$
8.8
$$

79.1

11.0

25. surrounded by chairs

$$
6.6
$$

59.3

33.0

26. is round

$$
11.0
$$

84.6

$4 \cdot 4$

27. Found in most buildings

$$
15.4
$$

79.1

$$
5.5
$$

28. holds waste

(qarbage)

0.0

19.8

80.2

29. is various colors 0.0

75.8

$$
3 \cdot 3 *
$$

30. used for decoration

$$
6.6
$$

78.0

31. found near

$$
\text { (under) desk } 15.4
$$

73.6

9.9

32. made of wood

30.8

67.0

2.2

33. has smooth

$$
\text { (finish) top } \quad 26.4
$$

72.5

1. 1

34. put things in

2.2

26.4

71.4

35. emptied when full

$2 \cdot 2$

13.2

84.6

36. mane of cushions

6.6

74.7

18.7

37. is smelly (dirty)

0.0

34.1

65.9

38. found against

$$
\text { (close to) a wall } 2.2
$$

86.8

11.0

39. is square

1. 1

90.1

7.7

40. is supportive 
41. usfr as a step
(stand on)
41.8
56.0
2.2

42. Found in libcaries

$$
16.5
$$

76.9

6.6

43. is dark-colored

0.0

44. is tall (high)

$4 \cdot 4$

45. a variety of styles

$$
37.4
$$

60.4

2.2

46. made of metal

6.6

47. is oval

5.5

48. has shelves

1. 1

49. used for studying

3.3

50. found in study room

$$
6.6
$$

89.0

85.7

$4 \cdot 4$

51. has a rouna top

9.9

52. is functional

(usefu1)

65.9

34.1

0.0

53. made of (covered

$$
\text { with) cloth }
$$

$4 \cdot 4$

87.9

7.7

54. used in homes

26.4

72.5

1.1

55. has $(1-4)$ legs

70.3

26.4

2. ?

56. found in rooms

30.8

69.2

0.0

57. is small (narrow)

7.7

58. used as a fontrest

$$
44.0
$$

54.9

1.1

59. is rectangular

3.3

85.7

11.0

60. holds trinkets 
(ornaments, candles)
1.1
24.2
74.7

61. is a variety of shapes

$\begin{array}{llll} & 35.2 & 59.3 & 4.4 \\ \text { 62. used for working } & 16.5 & 74.7 & 8.8 \\ \text { 6.3. is a furniture } & 85.7 & 13.2 & 1.1\end{array}$




\section{WASTEBASKET $(N=76)$}

Fssential Non-essential Not a property

1. made of plastic

10.5

89.5

0.0

2. found in kitchen

2.1. 1

43.3

55.3

3. WSEd for sitting

on (or at)

4. is comfortable

5. has flat surface

6. is a container

7. holds objects

8. has tall legs

9. is short

10. a variety of sizes

11. is expensive

12. is a type of seat.
19.7

0.0

53.9

22.4

7.9

16. has no back

17. holds (old)
56.6

17.1

75.0

40.8

89.5

77.6
55.3

73.7

25.0

1.3

9.2

71.1

5.3

42.1

35.5

19. used for writing 1.3

14.5

3.9

5.3

$14 \cdot 5$

22. is cylindrical 
23. holds objects

57.9

36.8

5.3

24. can be uncomfortahle

$$
0.0
$$

35.5

63.2

2.5. surroundad by chairs

$1 \cdot 3$

26. is round

6.6

27. found in most buildings

$$
57.9
$$

40.8

1.3

23. holds waste

$$
\text { (garbage) } \quad 94.7
$$

5.3

0.0

29. is various colors 39.5

30. usea for decoration

$$
1.3
$$

64.5

34.2

31. found near

$$
\text { (under) desk } \quad 34.2
$$

32. made of wood

5.3

33. has smooth

$$
\text { (finish) top }
$$

$$
0.0
$$

34. put things in

35. emptied when full 80.3

36. mate of cushions 0.0

37. is smelly (dirty) 14.5

38. Found against.

$$
\text { (close to) a wall } 13.2
$$

81.6

5.3

39. is square

1.3

82.9

13.2

40. is supportive

40.8

53.9 
41. Used as a step

$$
\text { (stand on) }
$$

$$
2.6
$$

60.5

36.8

42. found in libraries

$$
19.7
$$

43. is dark-colored

45. a variety of styles

$$
50.0
$$

46. made of metal

47. is oval

48. has shelves

0.0

13. 2

86.8

49. used for studying

0.0

22.4

77.6

50. found in study room

$$
32.9
$$

$64 \cdot 5$

$$
\text { 2. } 6
$$

51. has a round top

2.6

65.8

31.6

52. is functiona 1

$$
\text { (useful) } 80.3
$$

19.7

$0.0 *$

53. mate of (covered

with) cloth 1.3

42.1

54. used in homes

55. has $(1-4)$ legs

$1 \cdot 3$

55. found in rooms

38.2

57. is small (narrow)

0.0

53. used as a footrest 1.3

59. is rectangular.

3.9

60. holds trinkets fornaments.
2.6
56.6

$42.1 *$

52.6

2. 6

$26 \cdot 3$

72.4

56.6

0.5

78.9

18.4

73.7

22.4

85.5

10.5 
page 161

61. is a variety of shapes

57.9

62. used for working 11.8

63. is a Eurniture 30.3
39.5

2.6

55.3

32.9

42.1

26.3 
CATEGORY 5

VEHICLF: $(N=168)$

Fssential Non-essential Not a property

1. made of metals

48.2

50.0

0.6

2. has wheels

73.2

25.0

0.6

3. means of

transportation

91.1

$8 \cdot 3$

0.6

4. is a machine

70.2

28.0

0.6

5. is noisy

6.0

91.7

1.8

6. is vacious colors 35.1

61.9

2.4

7. is drivon

72.6

25.6

1. 2

8. has radio

8.9

86.3

4. 2

9. is expensive

12.5

82.7

3.6

10. has stecring wheel 57.7

37.5

1.8

11. Found in Eucope 11.9

79.2

$8 \cdot 3 *$

12. is various sizes 47.6

50.0

1.8

13. neers a conductor 36.9

50.6

11.3

14. is not-common

3.6

37.5

85.3

15. takes gas

37.5

58.3

1.8

16. needs a face

5.4

82.1

11.9

17. is fun

13.1

72.6

13.1

18. has seats

58.9

39.3

0.6

19. is crowded

2.4

$8.3 \cdot 9$

13.1

20. is various shapes 48.8

48.2

1.8

21. has trunk

20.8

73.8

4. 8

22. has sharp blades 4.2

48.2

47.0

23. has engine (motor) 53.6

41.7

4. 2 
2.4. is pusher with

one foot

25. has a bell

26. is dangerous

(accidents)

27. chilàren's toy

28. is black

29. has laces

30. has hrakes

31. is a pair

32. is open

33. is old-fashioned

34. has lights

35. has steering

$$
\text { hanỏle }
$$

36. Found in cities

37. is fast

38. has metalic frame

39. is a necessity

22.6

8.9

40. used for sport

41. has rubber tires 46.4

42. used on ico

1.8

4.3. is various makes

41.7

44. needs repairs

36.9

45. is shoe like

4.6

46. carries peopje
61.9

30.4

69.6

26.8

76.8

$8.3 *$

57.1

38.7

26.8

32.7

30.4

75.6

79.8

51.8

41.1

10.1

71.4

2.6

69.0

22.0

$17.9 *$

3.0

4.8

3.6

2.4

82.1

2.4

54.8

59.5

16.7

84.5

5.4

51.2

1.8

67.9

29.8

56.5

1.2

57.1

5.4

31.5

62.5

(passengers)

57.7
41.1

0.6 
47. has horn

27.8

48. can glid?

$7 \cdot 1$

49. has ( 2 to 4$)$ doors 35.1

50. is a symbol of prestige.

$$
\text { (status) }
$$

12.5

51. is white

1.2

52. has hubcaps

19.0

53. is popular in winter

$$
4.8
$$

54. is luxurious

4.5

55. used Eor hockey

0.0

56. is various stylos 45.2

57. made of leather

1.2

58. has qlass windows 33.3

59. is fun for cruising

$$
\text { 13. } 1
$$

60. goes on tracks

$$
6.0
$$

61. found in San

$$
\text { Francisco }
$$$$
7.1
$$

62. covers aistance

64.9

63. qoes on roads (hiqhways,

driveways)

53.6
75.6

79.2

$$
\text { 7. } 1
$$

76.8

16.7

67.3

2.4

20.2

2. 4

13.1

8.9

3.6

19.6

8.3

$61.9 *$

3.6

45.8

3. 0

62.5

8.9

1.8

31.5

42.9

1.8 


\section{CAR ( N $=79)$}

Essential. Non-essential Not a property

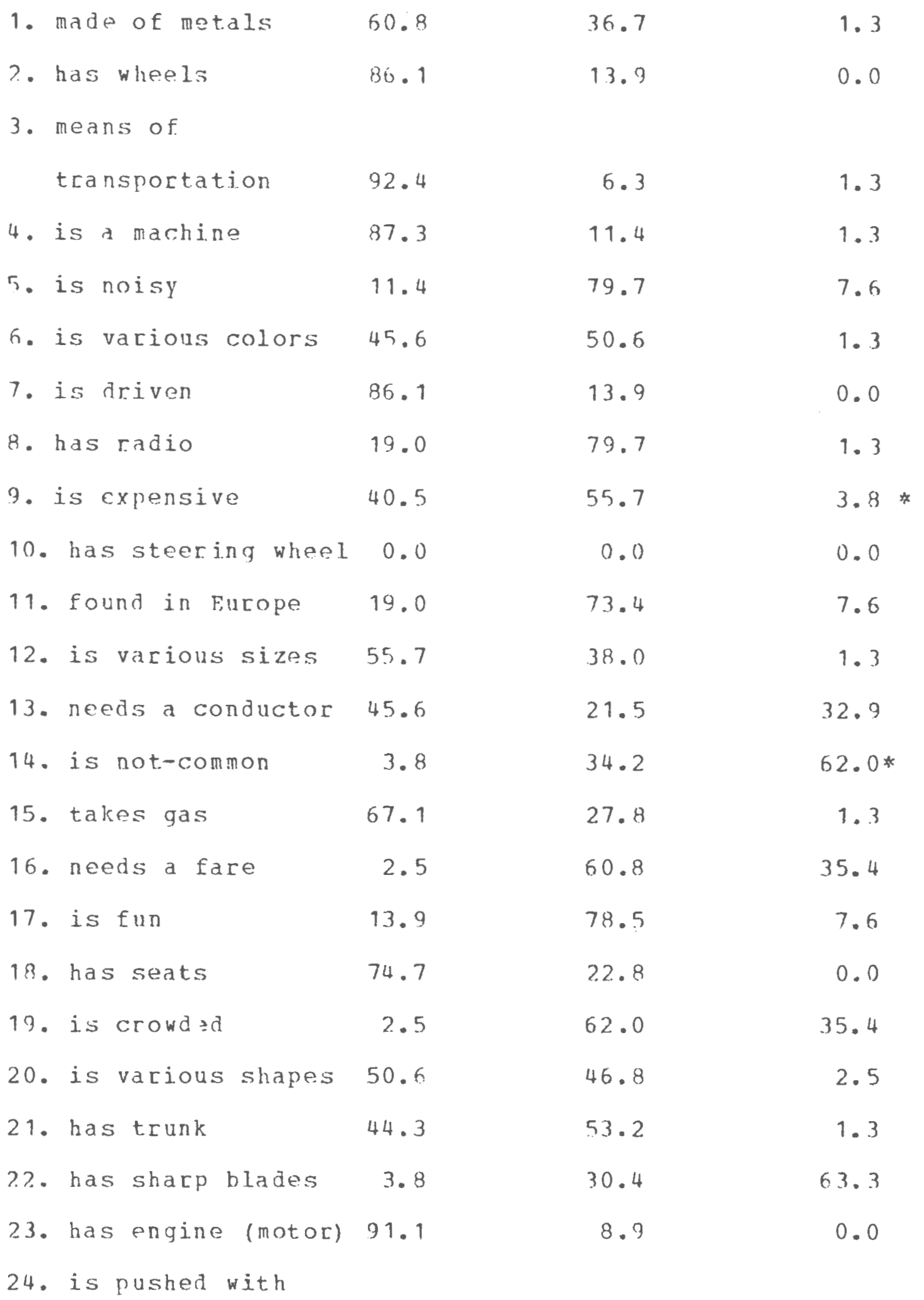


one foot

25. has a bell

26. is dangerous

(accidents)

27. children's toy

28. is hlack

29. has Iaces

30. has brakes

31. is a pair

32. is open

33. is old-fashioned

3. 8

62.0

34. has lights

35. has steering

handle

77.2

36. found in cities

37. is fast

38. has metalic frame

39. is a necessity

40. used for sport

41. has rubber tires

42. used on ice

43. is various makes

44. needs repairs

45. is shoe like

46. carries people

47. has horn

3. 8

51.9

41.8

3.8

$$
\text { (passengers) }
$$

89.9

49.4
16.5

41.8

67.1

7.6

58.2

11.4

88.6

2.5

$91.1 *$

21.5

68.4

35.4

26.6 *

2. 5

13.9

7.6

68.4

3.8

78.5

$7.6 *$

38.0

3.8

55.7

15.2

77.2

11.4

24.1

0.0

30.4

68.4 *

46.8

1.3

54.4

2.5

12.7

83.5
8.9

1.3

49.4 
48. can qlide

$$
3.8
$$

49. has (2. to 4) doors

$$
67.1
$$

31.6

$1 \cdot 3$

50. is a symbol of prestige

$$
\text { (status) }
$$

12.7

51. is white

0.0

52. has hubcaps

30.4

53. is popular in winter

$$
17.7
$$

63.3

29.0

54. is luxurious

$$
8.9
$$

58. has glass windows 50.6

59. is fun for cruising

$$
2.5
$$

60. goes on tracks

7.6

61. found in $\operatorname{san}$

Francisco

62. covers distance

$82 \cdot 3$

63. goes on roads (highways.

$$
\text { driveways) }
$$

$$
87.3
$$

64. is a vehicle

70.9

65. has steering

$$
\text { handle }
$$

$$
70.9
$$

83.5

15.2

44.3

55.7

49.4

39.2

58.2

7.6

83.5

1. 3

43.0

0.0

$\begin{array}{ll}25.3 & 2.5\end{array}$

16.5

1. 3

10.1

1.3

21.5

7.5

7.6 
TROLLF:Y ( $N=75)$

Fssential Non-essential Not a property

1. made of metals

2. has wheels

3. means of

transportation

4. is a machine

5. is noisy

6. is various colors

7. is driven

8. has radio

9. is expensive

10. has steering wheel 62.7

11. found in Europe 13.3

12. is various sizes 32.0

13. needs a conductor 80.0

14. is not-common

15. takes qas

16. nceds a fare

17. is fun

18. has seats

19. is crowded

20. is various shapes

21. has trunk

22. has sharp blades

23. has engine (motor)

89.3

65.3

36.0

25.3

32.0

57.3

9.3

68.0

25.3

4.0
60.0
40.0
0.0
8.0
5.3

9.3

1. 3

29.3

5. 3

64.0

0.0

64.0

1. 3

18.7

9.3

58.7

36.0

68.0

12.0

15.0

20.0

74.4

9.3

61.3

6.7

20.0

0.0

60.0

14.7

24.0

42.7

41.3

$1.3 *$

77.3

13.3

32.0

0.0

70.7

4. 0

48.0

20.0

37.3

57.3 *

26.7

68.0 
24. is pushed with

$$
\text { one foot }
$$

25. has a bell

26. is dangerous

(accidents)

27. children's toy

2.8. is black

29. has laces

30. has rrakes

31. is a pair

32. is open

33. is old-fashioned

34. has lights

35. has steering

handle

36. found in cities

37. is fast

38. has metalic frame

39. is a necessity

40. used for sport

41. has rubber tires

42. used on ice

43. is various makes

44. nfeds repairs

45. is shoe like

46. carries people

$$
2.7
$$

58.7

8.0

1.3

1.3

1.3

90.7

1.3

21.3

37.3

53.3

76.0

52.0

20.0

58.7

13.3

0.0

8.0

0.0

34.7

38.7

4.0
17.3

$80.0 *$

41.3

0.0

68.0

24.0 *

70.7

16.0

84.0

0.0

80.0

16.0

56.0

6.7

44.0

1. 3

14.7

8.0

45.3

2.7

68.0

12.0

37.3

4.0

61.3

25.3

30.7

69.3

25.3

65.3

14.7

85.3

62.7

2.7

52.0

$9.3 *$

8.0

86.7

0.0 

47. has horn
30.7
48.0
21.3
48. can glide
16.0
45.3
38.7
49. has ( 2 to 4$)$ doors 20.0
49.3
30.7

50. is a symbol of prestige (status)

0.0

18.7

81.3

51. is white

1.3

86.7

12.0

52. has hubcaps

2.7

42.7

54.7

53. is popular in sinter

54. is luxurious 0.0

48.0

48.0 *

55. used for hockey

58.7

41.3

56 . is various styles 34.7

13.3

84.0

57. made of leather

58. has qlass windows 26.7

61.3

12.0

59. is fun for cruising

$$
13.3
$$

60.0

26.7

60. goes on tracks

86.7

12.0

0.0

61. found in San

Francisco

62. covers distance

42.7

69.3

63. goes on roads (highways,

$$
\text { driveways) }
$$

13.3

64. is a vehicle

93.3
$29 \cdot 3$

56.0

5.3

1.3 
SCOOTER $(N=91)$

Essential Non-essential Not a property

1. made of metals

47.3

45.1

5.5

2. has wheels

33.4

$4 \cdot 4$

1. 1

3. means of

transportation

74.7

18.7

3.3

4. is a machine

51.6

27.5

20.9

5. is noisy

6.6

66.1

23.1

6. is various colors 35.2

62.6

2.2

7. is driven

75.8.

15.4

8.8

8. has radio

2.2

27.5

70.4

9. is expensive

0.0

65.9

34.1

10. has steering wheel 1.1

39.6

20.9

11. Found in Furope

7.7

82.4

8.8

12. is various sizes 47.3

49.5

2.2

13. needs a conductor 33.0

26.4

38.5

14. is not-common

2.2

73.6

24.2

15. takes gas

$14 \cdot 3$

24.2

61.5

16. needs a fare

0.0

16.5

81.3

17. is fun

30.8

65.9

3.3

19. has seats

17.6

37.4

45.1

19. is crowdad

0.0

31.9

65.9

20. is various shapes 38.5

44.0

17.6

21. has trunk

0.0

15.4

84.6

22. has sharp blades 0.0

25.3

73.6

23. has engine (motor) 14.3

33.0

52.7

24. is pusher with 


$$
\text { one foot }
$$

25. has a bell

26. is dangerous

(accidents)

27. children's toy

28. is black

29. has laces

30. has trakes

31. is a pair

32. is open

33. is old-fashioned

13.2

34. has 1ights

35. has steering

handle

36. found in cities

84.6

5.5

13.2

37. is fast

38. has metalic frame

40.7

39. is a necessity

0.0

40. used for sport

18.7

41. has rubber tires

46.2

42. used on ice

26.4

43. is various makes

34.1

44. needs repairs

45. is shoe like

46. carries people

$$
\text { (passengers) }
$$

47. has horn

26.4

5.5

50.5

9.9
23.1

$5 \cdot 6$

70.3

24.2

69.2

13.2

48.4

7.7

82.4

17.6

17.6

$81.3 *$

25.3

36.3

12.1

87.9

22.0

5.5

75.8

11.0

48.4

37.4

7.7

7.7

79.1

13.2

72.5

12. 1

51.6

7.7

54.9

41.8

62.6

18.7

45.1

7.7

71.4

1. 1

54.9

9.9

67.0

5.5

25.3

69.2

37.4

12. 1 *

67.0

23.1 
49. can glide

$$
6.3 .7
$$

24.2

16.5

12.1

49. has (2 to 4) doors 0.0

50. is a symbol of prestige (status)

$$
4 \cdot 4
$$

51.6

42.9

51. is white

0.0

79.1

19.8

52. has hubcaps

4. 4

$47 \cdot 3$

48.4

53. is popular in winter

$$
0.0
$$

31.9

67.0

54. is luxurious

1. 1

55. used for hockey

0.0

56. is various styles 28.6

57. make of leather

1.1

58. has glass windows 1.1

59. is fun for cruising

$$
39.6
$$

60. goes on tracks

1. 1

61. found in $\operatorname{san}$

Francisco

4.4

65.9

62. covers distance

63. goes on roads (highways.

driveways)

47.3

64. is a vehicle

73.6
52.7

7.7

48.4

49.5

15.4

84.6

60.4

7.7

23.1

$75.8 *$

17.6

$18 \cdot 3$

20.9

76.9

78.0

15.4

30.8

1. 1

45.1

6.6

20.9
4. 4 


\section{SKATES $(N=76)$}

Fssential Non-essential Not a property
1. made of metals
17.1
59.2
22.4
2. has wheels
23.7
26.3
48.7

3. means of

transportation

42. 1

42. 1

14.5

4. is a machine

1.3

7.9

89.5

5. is noisy

2.6

30.3

$67: 1$

6. is various colors 21.1

76.3

2.6

7. is driven

5.3

11.8

80.3

8. has radio

0.0

2.6

97.4

9. is expensive

1.3

76.3

21. 1

10. has steering wheel

1.3

1.3

97.4

11. found in Europe

7.9

82.9

7.9

12. is various sizes 59.2

36.8

2.6

13. nefds a conductor 3.9

10.5

80.3

14. is not-common

1.3

50.0

$47.4 *$

15. takes gas

1.3

0.0

98.7

16. needs a Eare

1. 3

$5 \cdot 3$

93.4

17. is fun

30.3

65.8

$3.9 *$

18. has seats

0.0

1.3

97.4

19. is crowded

2.6

1.3

96.1

20. is various shapes 26.3

48.7

25.0

21. has trunk

1.3

0.0

98.7

22. has sharp blades 64.5

34.2

1.3

23. has engine (motor) 0.0

$2 \cdot 6$

97.4

24. is pushed with 

one foot
6.6
43.4
50.0

25. has a bell

1.3

28.9

68.4

26. is dangerous

$$
\text { (accidents) }
$$

9.2

75.0

15.8

27. children's toy

11.8

71.1

14.5

23. is black

3.9

89.5

6.6

29. has laces

60.7

30.3

0.0

30. has brakes

1.3

10.5

$88.2 *$

31. is a pair

84.2

14.5

0.0

32. is open

1.3

27.6

69.7

33. is old-fashioned

3.9

50.0

44.7

34. has 1 ights

0.0

2.6

97.4

35. has steering

handle

36. found in cities

37. is fast

38. has metalic frame 21.1

39. is a necessity

1.3

40. used for sport.

81.1

41. has rubber tires 1.3

42. used on ice

64.5

43. is various makes

47.4

44. needs repairs

26.3

45. is shoe like

78.9
1.3

80.3

56.6

46.1

34.2

$28 . ?$

17. 1

35.5

50.0

56.6

21.1
97.4

17.1

1. 3

32.9

64.5

0.0

81.6

0.0

2. 6

15.8

0.0

46. carries people 
48. canglid? 56.6

49. has (2 to 4 ) doors 0.0

50. is a symbol of prestige (status)

3. 9

51. is white

5.3

52. has hubcaps

0.0

53. is popular in winter

$$
56.6
$$

54. is luxurious

2.6

55. used for hockey

7.3. 7

56. is various styles 42.1

57. made of leather

31.6

58. has glass windows

0.0

59. is fun for cruising

$$
21.1
$$

60. goes on tracks

1.3

61. found in $\operatorname{San}$

$$
\text { Francisco }
$$

62. covers distance

63.2

63. goes on roads (highways.

$$
\text { driveways) }
$$

10.5

64. is a vehicle
36.8

5. 3

0.0

98.7

26.3

69.7

86.8

7.9

2.6

97.4

43.4

0.0

46.1

51.3

2.5 .0

1.3

50.0

$5 \cdot 3 *$

61.8

6.6

3.9

96.1

52.6

26.3

14.5

84.2

76.3

19.7

34.2

2.6

47.4

42.1

9.2

68.4 
TABLE 15

Newman-Keuls Test for Instances at $F_{7} C_{1}$

$\begin{array}{lllll}I_{3} & C_{n} & I_{1} & I_{2} & I_{4}\end{array}$



$\begin{array}{lllllllll}I_{3} & 2.63 & -- & .02 & .27 & 1.18^{\star} & 2.58^{*} & 5 & 0.98\end{array}$

$\begin{array}{llllllll}C_{n} & 2.65 & - & .19 & 1.16 * & 1.56 * & 4 & 0.93\end{array}$

$\begin{array}{lllllll}\mathrm{I}_{1} & 2.84 & - & .97 & 1.37^{*} & 3 & 0.87\end{array}$

$\begin{array}{llllll}\text { I2 } & 3.81 & - & .40 & 2 & 0.77\end{array}$

$I_{4} \quad 4.21$

*indicates significant mean pairs 


\section{TABLE 16}

Newman-Keuls Test for Instances at $\mathrm{F}_{1} \mathrm{C}_{2}$

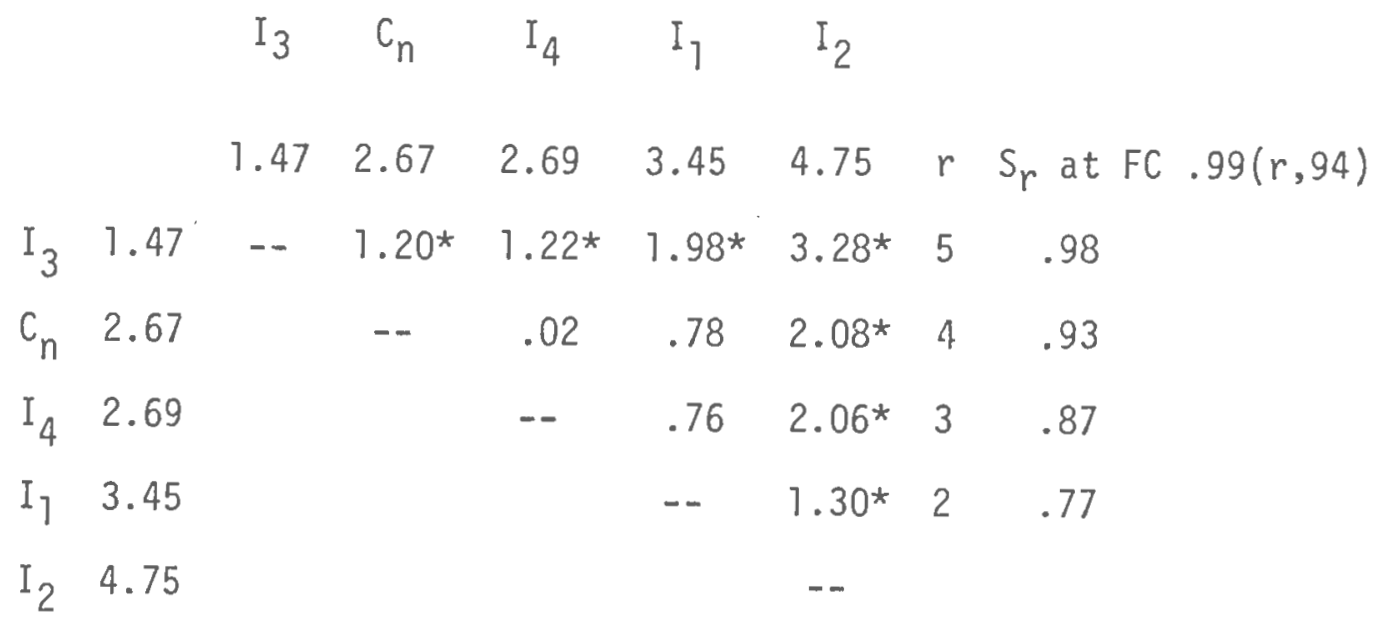

*indicates significant mean pairs 


\section{TABLE 17}



*indicates significant mean pairs 
TABLE 18

Newman-Keuls Test for Instances at $\mathrm{F}_{7} \mathrm{C}_{5}$

$$
\begin{array}{lllll}
C_{n} & I_{3} & I_{2} & I_{4} & I_{1}
\end{array}
$$

$\begin{array}{lllllll}2.62 & 2.86 & 3.19 & 3.27 & 5.18 & r & S_{r} \text { at } F C .99(r, 94)\end{array}$

$\begin{array}{lllllllll}\mathrm{C}_{n} & 2.62 & -- & .24 & .57 & .65 & 2.56 * & 5 & .98 \\ \mathrm{I}_{3} & 2.86 & & -- & .33 & .41 & 2.32 * & 4 & .93 \\ \mathrm{I}_{2} & 3.19 & & & -- & .8 & 1.99 * & 3 & .87 \\ \mathrm{I}_{4} & 3.27 & & & & -- & 1.91 * & 2 & .77 \\ \mathrm{I}_{1} & 5.18 & & & & & -- & & \end{array}$

*indicates significant mean pairs 


\section{TABLE 19}

Defining, Characteristic and Other Features Given as First Response for Categories and Instances
Defining
Characteristic
Others

Observed
Expected
$x^{2}=1,091.21$
$d f=2$
$p<.001$

1503.00

665.00

207.00

791.66

791.66

791.66

TABLE 20

Comparison of Defining, Characteristic and Other

Features Given as the First Feature for

Average of Instances and Category Names

Defining Characteristic 0thers

$\begin{array}{lrrr}\text { Instances } & 320.75 & 130.25 & 24.00 \\ \text { Categories } & 220.00 & 144.00 & 111.00 \\ & & & \\ x^{2}=115.64 & & \\ d f=2 & & \\ p<.001 & & & \end{array}$




\section{TABLE 21}

Comparison of Defining, Characteristic and Other Features Given as First Response for Levels of Relatedness and Categories

Defining Characteristic Others

Leve1 1

Instance 1

Category

Leve1 2

Instance $2 \quad 306$

Category

Level 3

Instance 3

312

Category

220

Level 4

Instance 4

306

142

144

144

159

144

120

144

Category

220
16

$\chi^{2}=182.57$

111

$p<.001$

10

$\chi^{2}=127.08$

$111 \quad p<.001$

43

$x^{2}=34.13$

$111 \quad p<.001$
27

$x^{2}=97.21$

$111 \quad p<.001$ 


\section{DISCUSSION: EXPERIMENTS 1 AND 2}

The results of this study did not support the hypothesis that the defining features of a word are more "basic" to word meaning than characteristic ones, as Smith, Shoben and Rips (1974) assume according to the criterion of total number of features generated. Defining features were significantly less rather than more frequently given than were characteristic features.

Such results can be readily interpreted through Collins and Quillian's network model, which assumes that each word has stored with it a configuration of pointers representing the word's meaning. The configuration contains superordinates and properties that uniquely characterize a word. It is assumed that under each node are stored the unique properties of the word, while properties shared with other concepts are stored under the superordinate set. Since defining features are shared with the category, it would be more difficult to retrieve them, because they are stored at a higher node than the characteristic features of a word that are stored exclusively with the word itself.

Analyzing the first feature generated by each 8 subject produced a different pattern. Defining features were more frequently given as first responses than characteristic or other idiosyncratic responses. This finding supports Smith's et al. (1974) assumption that defining features are more "basic" to word meanings. It was also found that there were more defining features given first for instances than for 
category names. Subjects showed greater difficulty in defining category names than instances and frequently they took refuge in enumerating the instances contained in a category (see Tables 20 and 21 for "other" responses). The emerging picture is that category names do not seem to function as conceptual superordinates and their representation in memory appears to be different than that of instances, at least to some extent. Additional evidence against the notion that category names function as subjective superordinates of their instances in semantic memory is discussed in Experiment 3 (page 193).

The hypothesis that the number of defining features in an item's meaning decreases with degree of abstractness was not supported in this study. For the five categories utilized in this experiment only five of the twenty instance words exhibited a significantly larger number of defining features than the corresponding category name. For the category "vegetable" two instances produced more defining features, for "bird", no instances produced more defining features and the other two categories each showed one instance producing more defining features. However, since this research utilized only two degrees of abstractness, category name and instance, the hypothesis cannot be conclusively rejected.

The total number of defining and characteristic features produced per word did not very with degree of relatedness. There appears to be something intrinsic about the words themselves that can account for the number of features produced. A cursory view of the Kučera and Francis (1967) word frequencies (see Table a) shows that they are not able to predict the total number of features produced for a word. They 
seem to be good predictors of high frequency words, when one would expect a substantial number of features, but not of low frequency words. Similarly, production frequencies, as measured by Battig and Montague (1969), cannot be hypothesized as the controlling factor of the produced features. For the most part, production frequency covaries with relatedness (see Table a) and it was found that relatedness cannot predict the number of features produced from memory. More specifically, the correlation between production frequency and number of defining features given as $r=.31\left(\omega^{2}=.09\right)$, while the correlation between production frequency and number of characteristic features given was $r=.57\left(\omega^{2}-.32\right)$.

A factor that might account for the number of features produced could be the absolute number of features required to express a word's meaning. Some words might need fewer features than others to exhaust their physical attributes. This factor rests upon the physical characteristics of what is denoted by word. If the words in a category are closely related in physical appearance (i.e., instances of the "bird" category), the features that can express their meaning should not differ greatly with degree of relatedness. On the other hand, if the words in a category are different in their physical description (i.e., table, stool, wastebasket) one would expect differing number of features. The same must be true for words between categories. Therefore, in order to test whether degree of relatedness, production frequency or any other interesting psychological variable can account for the number of defining and characteristic features produced, the number of features used to describe a word must be kept constant. Indeed, the 
methodology used in this experiment can be regarded as a procedure for identifying what features a word does describe. 


\section{EXPERIMENT 3}

Most traditions of thought, when describing subjective, semantic categories analytically, imply that categories are logical, clearly bounded entities, whose membership is defined by an item's possession of a simple set of criterial features. Category membership is, thus, an all-or-none phenomenon, as all instances possessing the criterial attributes have a full and equal degree of membership.

In contrast to such a view, it has been recently argued (Lakoff, 1972; Rosch, 1973) that many natural categories, (i.e., concepts designatable by words in natural languages) appear to possess an analog structure. Rosch $(1973,1975)$ has further characterized some natural analog categories as internally structured in terms of a prototype (clearest cases, best examples) of the category, surrounded by other instances of decreasing similarity to the prototype and of decreasing degree of membership.

Lakoff (1972) hypothesized that the structure of analog categories can be explained by incorporating not only a single criterial set of features (defining), but also a variable, incidental set (characteristic). Category membership, then, depends on the specific set of features that the category and its instances share. More specifically, it was hypothesized that best members share with the category both sets of features, while better non-prototypic members share only defining features and poorer non-prototypic members share only characteristic features, and it was this hypothesis which was 
addressed in Experiment 3.

In addition, Experiment 3 was designed to offer direct confirmation of the characteristic-features assumption. This assumption could be tested, since features were generated by subjects in the prior experiment for instances that varied in degree of relatedness to the category. However, these features were gathered through recall from memory. It has well been documented that a subject can recognize items much more easily than he can recall them (Postman and Rau, 1957; Shepard, 1967). This phenomenon could explain the fact that there were not very many features per word recalled by subjects and that the agreement among subjects about what features characterized the stimulus words was relatively low (see Table 1). In light of these results, the present experimenter decided that the characteristic features assumption could be more sensitively tested if frequencies of defining and characteristic features per word were obtained through recognition memory.

The effect of presenting the category name prior to vs. after its instances was also tested in this experiment. It was hypothesized that if category names are logical entities that share with their instances a criterial set of features and if category names function as superordinates, category names could trigger this criterial set, resulting in a greater number of features of the instances judged "essential" or "non-essential" than "not a property" after seeing the category name. However, if categories are of an analog structure, the presentation of category name prior to its instances should not effect the judgment of subjects in categorizing features. 
METHOD

\section{Subjects}

One hundred and sixty seven volunteer students from several Introductory Psychology classes at the University of Rhode Island participated in this study. The restrictions for participating were that (a) they had not participated in the previous experiments and (b) they should be native speakers of English. All subjects received extra credit towards course grade for their participation in this task. It was not expected that such reward can influence the subjects' responses in a systematic way as there are no right or wrong answers. For reasons described in the Method section of Experiment 1, age and sex of the subjects were not treated as variables.

Stimuli

Five sets of features, one set per category, were generated by pooling together the features produced by $10 \%$ or more subjects in Experiment 1 for a category name and all its related instances.

Due to the large number of features contained in each set, each subject received only two instances per category along with the category name. The instances included per category were separated by one degree of relatedness, so that a subject could either have instances of a category representing only Level 1 and 3 or 2 and 4 of relatedness. Among categories subjects could have Level 1 and 3 for some categories and 2 and 4 for others and presentation of specific instances were selected from 10 random orders.

A11 five category names were presented to each subject. For 
half of the subjects the category names were presented prior to all the instances (Group A) and for the other half were presented after all the instances (Group B). The order of category names presentation was selected from 10 random orders, each of which was represented equally in each group.

Procedure

Subjects were tested in their classes in groups. They were presented with a booklet that contained five category names and 10 instances, each with their set of features after the stimulus word. A typewritten set of instructions was given to each subject.

Subjects were asked to read each feature (carefully) and indicate with a check sign whether it belonged into one of three categories: (1) properties that are essential for the meaning of a word; (2) properties that are not absolutely necessary for the meaning and the word, but which further explain it; and (3) those that are not properties of the particular word. This third category was included to avoid forced choices especially for features of instances, since the features presented to $\underline{S}$ for each instance within a category (as well as the category name) were drawn from a common pool generated by all of those instances in the prior experiment.

Results

Chi-squares on the distribution of frequencies per feature on essential, non-essential and not-a-property categories, for category names preceding vs. succeeding instances were performed. One thousand five hundred and eighty chi-squares were computed of which 70 were significantly beyond the .05 level of confidence. Since one would ex- 
pect 79 of these chi-squares to be significant at the .05 level merely by chance, the accounted variability was deemed too small to allow a conclusion of any difference between the frequency distributions of the two groups. Thus, the frequencies of both groups were averaged and total percentages are presented in Table 14. As in Table 1 and 2 a category name is presented first followed by its instances arranged in a decreasing order of relatedness. For comparison purposes, features for instances were re-ordered to resemble the order of features of their category names. Significant chi-squares on the distributions of frequencies on essential, non-essential and not-a-property per feature, when category names were presented prior and after instances are indicated in Table 14 by an asterisk (*) next to the total percentages. Chi-squares were also performed from $3 \times 3$ contingency tables on the number of features that were judged as essential, non-essential and not-a-property for a category name vs. each of its instances. Subjects were also averaged in these analyses. A11 $20 \mathrm{chi}$-squares were found to be statistically significant and contingency co-efficients decreased with decreasing degrees of relatedness in all except four cases (see Table 22). Level 3 of relatedness for category "fruit" and furniture had a higher contingency co-efficient than Level 2, but not Level 1 . Also, relatedness Level 4 for category "fruit" was higher than Level 3 and 2 , but not from Level 1 .

Since the percentages of features judged "not-a-property" category were stable for all comparisons (being very low for the category name and more frequent but still low for instances) chi-squares were performed only on essential and non-essential categories for number of 
Chi-squares and Contingency Coefficients on the Number of Essential, Non-Essential and Not a

Property Features Shared by a Category Name and Its Instances ${ }^{* 1}$

\begin{tabular}{|c|c|c|c|c|}
\hline Variable & $x^{2}$ & $\underline{d f}$ & $p$ & $\begin{array}{l}\text { Contingen } \\
\text { Coeffici }\end{array}$ \\
\hline Vegetable by Spinach & 1609.07 & 4 & $<.001$ & 0.47 \\
\hline Vegetable by Tomato & 1286.19 & 4 & $<.001$ & 0.46 \\
\hline Vegetable by Parsley & 1005.84 & 4 & $<.001$ & 0.38 \\
\hline Vegetable by Dandelion & 127.18 & 4 & $<.001$ & 0.16 \\
\hline Fruit by Plum & 1040.15 & 4 & $<.001$ & 0.42 \\
\hline Fruit by Watermelon & 368.74 & 4 & $<.001$ & 0.29 \\
\hline Fruit by Gooseberry & 487.37 & 4 & $<.001$ & 0.32 \\
\hline Fruit by Avocado & 496.89 & 4 & $<.001$ & 0.33 \\
\hline Bird by Sparrow & 1160.97 & 4 & $<.001$ & 0.47 \\
\hline Bird by Parrot & 1285.76 & 4 & $<.001$ & 0.46 \\
\hline Bird by Vulture & 538.75 & 4 & $<.001$ & 0.34 \\
\hline Bird by Penguin & 305.98 & 4 & $<.001$ & 0.25 \\
\hline Furniture by Table & 1638.14 & 4 & $<.001$ & 0.48 \\
\hline Furniture by Bookcase & 612.04 & 4 & $<.001$ & 0.34 \\
\hline Furniture by Stool & 1090.48 & 4 & $<.001$ & 0.4 \\
\hline Furniture by Wastebasket & 439.18 & 4 & $<.001$ & 0.2 \\
\hline Vehicle by Car & 2948.11 & 4 & $<.001$ & 0.6 \\
\hline Vehicle by Trolley & 1088.64 & 4 & $<.001$ & 0.44 \\
\hline Vehicle by Scooter & 1095.79 & 4 & $<.001$ & 0.4 \\
\hline Vehicle by Skates & 346.61 & 4 & $<.001$ & 0.2 \\
\hline
\end{tabular}

${ }^{* 1}$ The set-up for each of these tables in its abstract form is:

Category

An Essential

Instance

${ }^{\star 2} C_{\max }=.82$

\begin{tabular}{|l|l|l|} 
Essential & Not-Essential & Not-a-Property \\
\hline $\begin{array}{l}\text { Number of } \\
\text { Features }\end{array}$ & & \\
\hline $\begin{array}{l}\text { Number of } \\
\text { Features }\end{array}$ & etc. & \\
\hline $\begin{array}{l}\text { Number of } \\
\text { Features }\end{array}$ & & \\
\hline
\end{tabular}


features shared by the category name and each instance, while averaging across categories for comparable levels of relatedness. Here it was even more clearly seen that degree of overlap as measured by $\phi$ coefficients decreased with decreasing levels of relatedness (see Table 23). Overall it was the very high percentages shared between features judged non-essential (characteristic) for both category name and instances that greatly accounted for the $\phi$ variance in coefficients. These percentages remain at a stable high level. The percentage of features judged essential for both category names and instances was consistently higher than the percentage of features judged essential for the category and non-essential for the instance (a critical comparison). Indeed, for instances at Level 1 of relatedness this percentage is the second highest in the table $(22.26 \%$ overa 11$)$. This percentage declines steadily, however, as the level of relatedness decreases.

\section{DISCUSSION}

In agreement with several studies performed by Rosch (Rosch, 1975; Rosch and Mervis, in press) it was found that natural categories possess some aspects of an analog structure. It was shown that category names presented prior to instances did not effect the judgment of subjects in categorizing features. This finding is congruent with the view that category names do not function as superordinates in memory, but can be represented by the best exemplars of the category. As Rosch (Rosch and Mervis, in press) well puts it:

"As speakers of our language and members of our culture, we know that a chair is a more reasonable exemplar of the category furniture than a radio, and that some chairs fit 


\section{TABLE 23}

Essential and Non-Essential Classes of Features for Category and Levels of Relatedness

\section{Category}




our idea or image of a chair better than others." (Rosch and Mervis, in press.)

The characteristic-features assumption received strong support from the fact that subjects categorized features into essential and non-essential with high agreement. Furthermore, properties attributed to specific kinds of features were also confirmed. Specifically, the hypothesis that relatedness norms reflect the structure of semantic categories which can be analyzed into specific kind(s) of features (defining and characteristic) was supported, as was the hypothes is that as relatedness measure of instance to category varies, so does the degree of overlap between feature classes. Contrary to the prediction that the correlation of defining and characteristic features of an instance with the defining and characteristic features of its category would vary with degree of relatedness of the instance to the category, it was found that the correlation held only for the defining features. Characteristic features remained constant at various degrees of relatedness, while the percentage of defining (essential) features shared between the category name and its instances decreased as relatedness decreased. It should also be noted that categories and instances share their features as essential and non-essential more than chance; that is, the overlap of essential-essential features was greater than essentialnon-essential; and the overlap of non-essential-non-essential features was greater than non-essential-essential.

The more specific predictions derived from Lakoff's theory (1972) were only partially supported. It was confirmed that highly related instances would share their defining and characteristic features 
with those of their category name. Moderately related instances shared the category name's characteristic features, but overlapped their defining features less than the percentage shared with the highly related instance, which is contrary to Lakoff's view that moderately related instances should show a correlation only between their defining features and those of the category. However, Lakoff's prediction that low related instances would show a correlation only between their characteristic features and those of their category name was supported.

In summary, the natural language categories utilized in this experiment can be described as possessing something of an analog structure. This analog structure and the degree of membership, as defined by relatedness norms, was depicted by shifts on the number of defining features shared between instances, representing different levels of relatedness, and the category name. As relatedness decreased, defining features decreased. Therefore, the characteristic-features assumption has received supporting evidence, while it only partially supported Lakoff's theory.

\section{GENERAL DISCUSSION}

The present research investigated the structure of semantic memory representations. More specifically, it addressed the question of what constitutes the cognitive representation of word meanings for category names and instances and whether such meanings can be represented by tivo sets of features, one being criterial and the other incidenta?. 
There were three basic findings from the present series of experiments. First, it appears that the psychological structure of natural language categories can be analyzed meaningfully into defining and characteristic sets of features. When subjects were asked to recall features that a word possesses they readily and reliably generated both types of features. This phenomenon offers support to the hypothesis that both sets constitute part of the meaning representation of a word. In fact, the number of characteristic features generated was greater than that of the defining features, which could be explained either by supposing that characteristic features are more basic to the meaning of a word, or that there are simply more characteristic features than defining features in the physical descriptions denoted by some words. Analyzing the first feature given, it was confirmed that defining features are more basic to word meaning than characteristic ones. Furthermore, subjects find it a meaningful task to separate features into defining and characteristic which provides face validity for such a distinction.

The second basic finding was concerned with the differential meaning representation of abstract and concrete words. It has been hypothesized that category names (perhaps all generic words) possess, for the most part, fewer defining features than concrete words like instances. This hypothesis was not supported and it was found that category names possess as many defining features as the instances of that category except for the highly related instances. Instances 1 and/or 2 possess a greater number of defining features than the category name. The mental representation of the category name seems to be 
exemplified by an ability to abstract general features from the instances of the category. The present results reveal that the category name cannot be represented by all members of the category but only through its best members. These results concur with other evidence in the field which supports the view that the mental representation of category names can best be exemplified by its best members (Rosch, 1975). However, the position supported by the present results needs further empirical evidence especially in view of the fact that only one degree of abstractness was utilized.

The third empirical finding confirmed that natural language categories possess an analog structure which defines degrees of membership through common features shared with the category name and its instances. A category name is represented by its best members and exemplars that share together a number of defining and characteristic features. As degrees of relatedness decrease, the overlap of defining features also decreases. Characteristic features, however, remain at a high level of overlap without demonstrating decreasing levels of relatedness. Since characteristic features, by definition, are not essential for the meaning of a word one would not expect them to depict fine discriminations among word meanings. Thus, while both defining and characteristic features constitute the structure of a category, only defining features depict degrees of relatedness among exemplars of that category.

It is very important that the pattern of features given by subjects and, therefore, raised to the awareness of the subjects, confirms the analog structure of categories as well as defining versus charac- 
teristic features distinction. A tendency of several experimenters has been to assume that meaning of words must reside in philosophical or linguistic primitives consisting of feature lists of that nature. To use Smith, Shoben and Rips' definition of features as an illustration, they assumed that a lexical item is represented by a set of relevant semantic dimensions (e.g., size, predacity, etc.). For each item, there is a distribution of possible values on each relevant dimension. At any given time, the meaning of a lexical item can be represented as a list of values, one value being sampled from each dimension. It is, then, these momentary values that Smith, et al. (1974) call features. Due to the hypothetical nature of these features, they were derived from raw data by multidimensional scaling techniques (Rips, Shoben and Smith, 1973). In contrast, the present research indicates that subjects can respond to the different features that define words and to the psychological reality of the relationship between whether or not a particular feature is needed for the definition of a word and whether or not it is needed to define that word's subordinate instances of varying degrees of typicality. 


\section{REFERENCES}

1. Anderson, J. R. and Reder, L. M., Negative judgments in and about semantic memory. Journal of Verbal Learning and Verbal Behavior, 1974, 13, 664-681.

2. Bierwisch, M. On classifying semantic features. In D. D. Steinberg and L. A. Jakobovits (Eds.), Semantics: An Interdisciplinary Reader in Philosophy, Linguistics and Psychology. Cambridge, England: University Press, 1971.

3. Bousfeld, W. A. The occurrence of clustering in the recall of randomly arranged associates. Journal of General Psychology, $1953,49,229-240$.

4. Brown, R. W. and D. McNeill. The "tip-of-the-tongue" phenomenon. Journal of Verbal Learning and Verbal Behavior, 1966, $\underline{5}$, 325-337.

5. Clark, H. H. Word Associations and Linguistic Theory. In J. Lyons (Ed.) New Horizons in Linguistics. Baltimore: Penguin, 1970.

6. Cofer, C. N. and Musgrave, B. S. (Eds.) Verbal Behavior and Learning: Problems and Processes. New York: McGraw-Hi 11 Book Company, 1963.

7. Collins, A. M. and Quillian, M. R. Retrieval time for semantic memory. Journal of Verbal Learning and Verbal Behavior, $1969,8,240-248$.

8. Collins, A. M. and Quillian, M. R. Facilitating retrieval from semantic memory: The effect of repeating part of an inference. Acta Psychologica, 1970a, $\underline{3}, 304-314$.

9. Collins, A. M. and Quillian, M. R. Experiments on semantic memory and language comprehension. In L.W. Gregg (Ed.) Cognition in Learning and Memory. Hew York: Wiley, 1970b.

10. Collins, A. M. and Quillian, M. R. Categories and subcategories in semantic memory. Paper presented at the annual meeting of Psychonomic Society, St. Louis, MO, 1971.

11. Conrad, C. Cognitive economy in semantic memory. Journal of Experimental Psychology, 1972, 92, 149-154.

12. Deese, J. The structure of associations in language and thought. Baltimore: Johns Hopkins Press, 1965. 
13. Deese, J. On the structure of associative meaning. Psychological Review, 1962, 69, 161-175.

14. Fillenbaum, S. and A. Rapoport. Structure in subjective lexicon. New York: Academic Press, 1971.

15. Freedman, J. L. and E. F. Loftus. Retrieval of words from Tong-term memory. Journal of Verbal Learning and Verbal Behavior, 1971, 10, 107-115.

16. Freedman, J. L. and E. F. Loftus. Retrieval of words from welllearned sets: The effect of category size. Journal of Experimental Psychology, 1974, 102, 1085-1091.

17. Glass, A. L. and K. J. Holyoak. The effect of "some" and "all" on reaction time for semantic decisions. Memory and Cognition, $1974,2,436-440$.

18. Glass, A. L. and Holyoak, K. J. Alternative Conceptions of Semantic Memory. Cognition, 1974/1975, 3(4), 313-319.

19. Glass, A. L., Holyoak, K. J. and O'De11, C. Production frequency and the verification of quantified statements. Journal of Verbal Learning and Verbal Behavior, 1969, $\underline{8}, 176-184$.

20. Grober, E. and Loftus, E. F. Semantic memory: Searching for attributes versus searching for names. Memory and Cognition, $1974, \underline{2}, 413-416$.

21. Holyoak, K. J. and GIass, A. L. The role of contradictions and counter-examples in the rejection of false sentences. Journal of Verbal Learning and Verbal Behavior, 1975, 14, 215-254.

22. Homa, D. Organization and long-term memory search. Memory and Cognition, 1973, I, 369-379.

23. Jenkins, J. J. and Palermo, D. S. Mediation processes and the acquisition of linguistic structure. In U. Bellugi and R. Brown (Eds.) The Acquisition of Language. Monographs of the Society for Research in Child Development, 1964, 29, (7), 141-169.

24. Johnson-Laird, P. N. Experimental psycholinguistics. Annual Review of Psychology, 1974, 25, 135-160.

25. Katz, J. J. Semantic Theory. New York: Harper and Row, 1972.

26. Kintsch, $w$. Notes on the structure of meaning in memory. In Tulving, E. and Donalson, W. (Eds.) Organization of Memory. New York and London: Academic Press, 1972. 
27. Kintsch, W. The representation of meaning in memary. Hillsdale, N. J.: L. E. Erlbaum Associates, 1974.

28. Lakoff, G. Hedges: A study in meaning criteria and the logic of fuzzy concepts. Papers from the eighth regional meeting, Chicago Linguistic Society, Chicago: University of Chicago Linguistics Department, 1972.

29. Landauer, T. K. and Freedman, J. L. Information retrieval from long-term memory: Category size and recognition time. Journal of Verbal Learning and Verbal Behavior, 1968, 7, 291295.

30. Loftus, E. F. Category dominance, instance dominance and categorization time. Journal of Experimental Psychology, 1973, 97, 70-74.

31. Loftus, E. F. and Bolton, M. Retrieval of superordinates and subordinates. Journal of Experimental Psychology, 1974, 102, $121-124$.

32. Loftus, E. F. and Cole, W. Retrieval attribute and name information from semantic memory. Journal of Experimental Psychology, $1974,102,1116-1122$.

33. Loftus, E. F. and Freedman, J. L. Effects of category-name frequency on the speed of naming an instance of the category. Journal of Verball Learning and Verbal Behavior, 1972, 11 , 343-347.

34. Loftus, E. F., Freedman, J. L. and Loftus, G. R. Retrieval of words from subordinate and superordinate categories in semantic hierarchies. Psychonomic Science, 1970, 21, 235-236.

35. Loftus, E. F. and Suppes, P. Structural Variables that Determine the Speed of Retrieving Words from Long-term Memory. Journal of Verbal Learning and Verbal Behavior, 1972, 11, 770-777.

36. Meyer, D. E. On the representation and retrieval of stored semantic information. Cognitive Psychology, 1970, I, 242-300.

37. Meyer, D. E. Correlated operations in searching stored semantic categories. Journal of Experimental Psychology, 1973, 99, 124-133.

38. Miller, G. A. A psychological method to investigate verbal concepts. Journal of Mathematical Psychology, 1969, ․․ 169-191.

39. Miller, G. A. English verbs of motion: A case study in semantic and lexical memory. In A. W. Melton and E. Martin (Eds.) Coding Processes in Human Memory. Washington, D.C.: Winston, 
1972.

40. Osgood, C. E. The nature and measurement of meaning. Psychological Bulletin, 1952, 49, 197-237.

41. Postman, L., and Rau, L. Retention as a function of the method of measurement. University of California Publications in

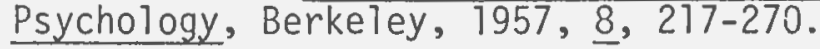

42. Quillian, M. R. Word concepts: A theory and simulation of some basic semantic capabilities. Behavioral Science, 1967, 12, 410-430.

43. Rips, L. J., Shoben, E. J. and Smith, E. E. Semantic distance and the verification of semantic relations. Journal of Verbal Learning and Verbal Behavior, 1973, 12, 1-20.

44. Rosch, E. On the internal structure of perceptual and semantic categories. In T. E. Moore (Ed.) Cognitive Development and the Acquisition of Language. New York: Academic Press, 1973.

45. Rosch, E. Linguistic relativity. In A. Silverstein (Ed.) Human Communication: Theoretical Perspectives. New York: HaTsted Press, 1974.

46. Rosch, E. Cognitive representations of semantic categories. Journal of Experimenta1 Psychology: General, 1975, 104, 192233.

47. Rosch, E. and Mervis, C. B. Family resemblances: Studies in the internal structure of categories. Cognitive Psychology, in press.

48. Sanford, A. J. and Seymour, P. H. K. The influence of response compatibility on a semantic classification task. Acta Psychologica, 1974, 38, 405-412.

49. Sapir, E. Grading: A study in semantics. Philosophy of Science, $1944,11,93-116$.

50. Schaeffer, B. and Wallace, R. The comparison of word meanings. Journal of Experimental Psychology, 1970, 86, 144-152.

51. Shepard, R. N. Recognition memory for words, sentences and pictures. Journal of Verbal Learning and Verbal Behavior, 1967, $\underline{6}, 156-163$.

52. Smith, E. E. and Haviland, S. E., Buckley, P. B. and Sack, M. Retrieval of artificial facts from long-term memory. Journal of Verbal Learning and Verbal Behavior, 1972, 11, 583-593. 
53. Smith, E. E., Shoben, E. J. and Rips, L. J. Structure and process in semantic memory: A featural model for semantic decisions. Psychological Review, 1974, 81, 214-241.

54. Tulving, E. Episodic and semantic memory. In E. Tulving and W. Donaldson (Eds.) Organization of Memory. New York: Academic Press, 1972.

55. Wilkins, A. T. Conjoint frequency, category size and categorization time. Journal of Verbal Learning and Verbal Behavior, $1971,10,38 \overline{2-385}$. 
APPENDIX I

REVIEW OF THE MAJOR SEMANTIC MEMORY MODELS

The Collins and Quillian Model

The major proponents and investigators of semantic, psychological network models are Collins and Quillian (1969). The model of semantic memory structure that they introduced was adopted from Quillian's $(1967,1969)$ computer simulation model, in which each word has stored with it a configuration of pointers to other words in memory. This configuration represents the word's meaning. Two general types of words are in a configuration: superordinates $(S)$ of a word (e.g. bird is a superordinate of canary) and properties ( $P$ ) which uniquely characterize the word (e.g. yellow is a property of canary). According to this model, memory is organized hierarchically and the amount of space needed for storage is minimized, because under each node are stored only the intrinsic properties of the concept, while properties shared with other concepts are stored under the superordinate set. Connections between the nodes specify particular relations that hold between the concepts. As Landauer and Meyer (1972) showed, two concepts like "collie" and "dog" would be represented at distinct nodes in the network and a link between these nodes would specify that "collies" are a subset of "dogs".

The model further proposes that semantic memory retrieval involves entering this structure and searching for connections between concepts. Retrieval time depends on the distance separating concepts 
and on the number of connections between them. For example, suppose that a person is asked to judge whether "a collie is a dog". The theory assumes that he would search the memory for an appropriate connection between "collie" and "dog". The search would take less than one to judge whether "a collie is an animal", because the semantic distance between "collie" and "dog" is less than between "collie" and "animal".

The supporting evidence for the greater semantic distance between "collie" and "animal" than "collie" and "dog" came from reaction time experiments. To verify that "a canary is a canary" took less time than to verify "a canary is a bird" which in turn took less time to verify than a "canary is an animal". The assumption was made that reaction time is an index of retrieval from long-term memory and measures semantic distance, which is the number of nodes, or concepts, separating the two concepts linked together in a given statement. The hierarchial retrieval of facts has received additional support by Collins and Quillian (1970a, 1972) and Smith and Haviland, Buckley and Sack (1972). One unexpected finding was that, although the closeness of items in the semantic network facilitated positive judgments about them, it impeded negative judgments. For example, it was more difficult to evaluate "a canary is an ostrich" than to evaluate "a canary is a fish". As Johnson-Laird (1974) reports, this difference has been repeatedly replicated (Schaeffer and Wallace, 1969, 1970; Wilkins, 1971) and perhaps constitutes the central regularity of semantic judgment. The greater the similarity in meaning between words the easier it is to make a positive judgment and the harder it is to make a 
negative judgment about a semantic relation between them. The network model provides a natural account for the facilitation of positive judgments, but to account for the negative judgments it is necessary to suppose that are impeded by extraneous links between neighboring words (Collins and Quillian, 1972).

The property of hierarchial model of memory was supported by differential reaction times to various feature or property statements. Collins and Quillian (1969) found that the mean reaction time for statements like "a canary has wings" to be greater than the mean reaction time for statements like "a canary is yellow". The property of wings is common to all birds and it is not unique to canary, so it takes more time to retrieve the properties of a higher node than of the same node.

However, this assumption of cognitive economy has been attacked by Conrad (1972). In her experiment, Conrad did not simply generate a system of properties as Collins and Quillian had done, but collected normative data for properties (Conrad, 1971). Replicating Collins and Quillian's experiment she confirmed that property (P) sentences with a low and moderate frequency took longer to retrieve from memory than those of high frequency, with the exception of the high frequency sentences pertaining to a property of a superset (e.g. a canary can fly). The high frequency $P$ sentences did not show a regular increase in reaction time with an increase in $P$, suggesting that these properties are stored with the words which they uniquely define and also with every word with which they are frequentiy associated. 
The Landauer and Freedman Model

Another model of semantic memory is presented by Landauer and Freedman (1968) and is based on the principle of a stored 1ist. Each category word in memory is assumed to have stored with it a "list" of al1 instances, or subordinates, known to the subject. If the statement "a collie is a dog" is presented for evaluation, the subject must search the "list" stored with "dog" as a concept name to discover the presence of "collie" as an instance. Similarly, if the statement "a collie is an animal" is presented for evaluation, the subject must search the "list" stored with "animal" as a concept name. This model differs from that of Collins and Quillian's in that it does not hypotesize a hierarchial network of interrelated concepts or nodes rather each word brings together its own list of superordinates and instances known to the subject.

Landauer and Freedman (1968) found that when common English words were asked to be classified as belonging or not belonging to well-known verbal categories, reaction time increased slightly as a function of category size, but only sometimes. Based on such results it was inferred that the search of list content involves parallel processing; that is, different entries in memory store are consulted simultaneously. Category size was manipulated by employing sets of nested categories, such as "word", "noun" or "living thing", "animal", "dog". The category size effect refers to the phenomenon of increases in category size, defined by the manipulation of category members, producing reaction time increases.

This category size effect was explained by Collins and Quillian 
(1970c) in terms of an inference process. For instance, people take less time to confirm a sentence like "a daisy is a flower" than to confirm a sentence like "a daisy is a plant". This difference is predicted by a theory that people confirm sentences like "a daisy is a plant" by inference from their knowledge that daisies are flowers and flowers are plants. Thus, the controversy between the two modeis is reduced to a category size effect. If such an effect exists, then, Landauer and Freedman's model is correct, while Collins and Quillian's mode 1 can be disputed.

\section{Category Size}

As was previously mentioned, category size effect was supported by Landauer and Freedman's results (1968) using a series of nested categories. Collins and Quillian (1969), Meyer (1970), and Landauer and Meyer (1972) using somewhat different procedures report that categorization took somewhat longer for large categories. In contrast, Neisser (1964) compared categorization time for "animal" and "first names" and found no difference in latency. Although most of these studies did find a small difference favoring the small category, the magnitude of the difference was very small relative to the size of the categories involved. Also Collins and Quillian (1970c) demonstrated the categorization time for instances of a certain category (positive instances) did not increase consistently across the "dog", "bird", and "animal" distinction as was expected. The results for negative instances were more complicated. They found that only sometimes took longer to reject a negative instance as belonging to a larger category. They explained their findings in terms of the confusability of concepts 
close together in semantic relatedness.

This relatedness effect has been documented by collins and Quillian (1970), Meyer (1970), Wilkins (1971) and Rips, Shoben and Smith (1973). However, Wilkins (1971) has presented additional data which suggest a standard category size effect even when relatedness is held constant, though flaws in his experimental design, as for example the way he equated similarity across categories, call his conclusions into question. Landauer and Meyer (1972) have attempted to hold relatedness constant and have also shown a category size effect. Thus, relatedness appears not to account for all of the category size effect. Freedman and Loftus (1971) departed from the previous work on categorization time and instead measured the speed with which subject could actually produce a word himself/herself. Subjects were presented with a noun category paired with either a letter (i.e. country-A) or an adjective (i.e. country-sma11) and were asked to provide a word belonging in the overlap by the pair. They repeatedly found that the speed of producing a word was independent of the size of the category to which the word belonged (Freedman and Loftus, 1971; Loftus, Freedman and Loftus, 1970). Furthermore, Freedman and Loftus (1974) demonstrated that reaction time increased as a function of size with small categories (less than 7 words), but at some point levelled off with no additional increase even with categories as large as 32 members. However, category size was manipulated through various lists of instances of newly constructed categories. The subjects were required to learn these 1 ists well and free recall as well as production of an instance while given another instance as a cue were tested. Due to their 
manipulation of category size effect and the fact that category size has not been confirmed as a psychological variable, Freedman and Loftus' (1974) results cannot be directly compared to the rest of the category size experiments in this area.

Smith and his associates (Smith and Haviland, Buckley and Sack, 1972) sought to solve the controversy between hierarchial and category size effect. In two experiments subject judged the truth or falsity of previously learned artificial facts, each fact consisting of a noun from a natural language hierarchy paired with an arbitrary digit, e.g. Bird-2. Experiment 1 used a two-level hierarchy and the results were consistent with Collins and Quillian's (1969) model. Experiment 2 used a three-level hierarchy and the results were inconsistent with Collins and Quillian's model, but rather supported a model in which all properties of a noun are stored with and retrieved from that noun (Landauer and Freedman's model, 1968). Comparable results were found by Conrad (1972) who found that when associative frequency among properties was held constant hierarchial effects were present for statements about supersets but absent for statements about the properties of items. In summary, these findings support that portion of the Collins and Quillian model of semantic memory which suggests that words are organized hierarchially in memory. On the other hand, the hypothes is of cognitive economy of storage has received little or no support. And an additional problem for a hierarchial model, as was previously mentioned, is that it cannot explain the reaction times for refuting negative statements.

Finally, Smith, Shoben and Rips (1974) found that category size, 
as manipulated by nested categories, had no significant effect on categorization time. However, when an index of the degree of similarity between the nested categories was obtained through production frequency norms, it was found that reaction time varied inversely with the degree of similarity, that is, when overall similarity increased, reaction time decreased while when overall similarity decreased, reaction time increased.

The Smith, Shoben and Rips Model

This mode1 emerges from a set-theoretic tradition in semantic memory research (Meyer, 1970; Schaeffer and Wallace, 1970). The first assumption about the meaning of a word is based on a semantic theory outlined by Lakoff (1972) which proposes that meaning is not an unanalyzable unit but rather can be represented as a set of semantic features. Smith et al. suggest that the features associated with a given category vary in the extent to which they define that category. In other words, there is a continuum along which some features will be more incidental or "characteristic" features. This variation in definingness plays a role in a categorization situation if accurate judgments are to be made. Specifically, the defining features must be given greater emphasis than the characteristic features. While the features for each item are presumed to be ordered from top to bottom in degree of definingness, there is presumed to be an explicit boundary distinguishing defining from characteristic features. The number of defining features contained in an item's meaning are assumed to decrease as the item becomes increasingly abstract.

Based on these assumptions the mechanisms of a categorization 
task, "a subject $(S)$ is a predicate $(P)$ " sentence were outlined. A semantic categorization is believed to require two distinct comparison stages. In the first stage, lists of features for the instance and category are retrieved, including features drawn from characteristic as well as defining dimensions, yielding a measure, $x$, of overall similarity. This measure takes into account the proportions of the category's dimensions which are shared by the test iten and the proximity of values (features) on each of these common dimensions. But as Smith et al. pointed out, it is difficult at this point to be more specific about the exact combination rule used to compute overall similarity. The $x$ value of overall similarity is compared to two preset criterial levels, one, a high level called $c_{1}$ and the other, a low level called $c_{0}$. If $x$ exceeds $c_{1}$, then a positive response (true) can be made, while if $x$ is less than $c_{0}$, a negative response (false) is executed. When $x$ falls in the intermediate range between $c_{0}$ and $c_{7}, a$ second comparison stage is necessary before a response can be determined. In this stage, only the defining features of instance and category are retrieved and compared. A positive response can be made if (a) each defining dimension of the category is also a defining dimension of the instance, and (b) the particular features on these dimensions which the instance possesses are within the range of allowable values for the category.

A number of predictions follow from this model: First, for any target category, "true" reaction time should decrease as the typicality of the test instance increases. Typicality, assumed to measure overall similarity, is a term introduced by Rosch (1973) who asked subjects to 
judge how good an example of a certain category various instances of that category were. This prediction has been partially supported by Smith (1967), Hilkins (1971), Loftus (1973), Rips, Shoben and Smith (1973) and Rosch (1973).

Second, for correct "false" response reaction time is predicted to decrease as relatedness decreases. Relatedness is hypothesized as measuring overall semantic similarity for "false" responses. However, the exact mechanism of measuring relatedness is not specified. This term, semantic relatedness, was introduced by Collins and Quillian (1970c) in an attempt to explain why some negative instances (i.e. tulip vs. dog or bird) took longer to be rejected than others (i.e. magnisium vs. dog or bird). He hypothesized that relatedness was a major variable. Therefore, instances that are closely related require more time than instances that are not closely related. Ratings of relatedness were obtained by Rips et al. (1973) who asked subjects to indicate the degree of relatedness of a standard word to a group of comparison words on a 4-point scale. A relatedness effect using this criterion has been partially documented by Collins and Quillian (1970c), Meyer (1970), Wilkins (1971) and Rips, Shoben and Smith (1973).

Considering the issue of category size, Smith et al. (1974) propose that in terms of the feature-comparison model, an increase in category size should affect both stages of their model, because of changes in the number of defining and characteristic features. Defining features are supposed to decrease as category size increases, because the terms become more abstract. Hence, if only the second stage is considered, an increase in category size should lead to a decrease in 
"true" reaction time. But as one increases category size, there might be a substantial change in the overall similarity which determines whether the second stage will need to be executed. An increase in overall similarity decreases "true" reaction time, while a decrease in overall similarity increases "true" reaction time. Moreover, since "false" reaction time is known to increase with relatedness, it follows that an increase in category size can lead to an increase in "false" reaction times.

These predictions for a category size effect were supported by Smith et al. (1974). In that study category size was manipulated through nested categories and a measure of the category's overall semantic similarity to the instance was indexed by production frequency as measured by Loftus and Scheff (1971). Loftus and Scheff obtained a set of norms by instructing a group of subjects to list three superordinates for each of 50 instances. To support the assumption that production frequency (as measured by Loftus and Scheff, 1971) is a measure of the category's overall semantic similarity to the instance, Smith et al. reported a correlation $r=.85(\mathrm{df}=10, p<.01)$ obtained by Rips et al. (1973) between ratings and production frequencies. However, that correlation was inappropriately obtained between Battig and Montague's norms (1969) of category-instance frequency and ratings of degree of relatedness on a 4-point scale between the standard word and a comparison word. Battig and Montague's norms were collected by reading category names to subjects and asking them to write down in 30 seconds as many members of each category as they could. Thus, Battig and Montague's norms measure instance dominance (the fre- 
quency with which an instance is given to a particular category) while Loftus and Scheff's norms measure category dominance (the frequency with which a superordinate is given to a particular instance). Loftus (1973) offered evidence that supported the differential nature of these measures. In a verification task requiring whether the instance was a member of a category, when the instance preceded the category (e.g. robin-bird) category dominance determined reaction time, but when the category preceded the instance (e.g. bird-robin) the instance dominance determined reaction time.

Smith et al. (1974) offered further evidence to support the assumption that production frequency measures semantic relatedness of the instance-category pair. Correlation was obtained for the difference score for ratings between statement pairs of the form "an $S$ is a $P_{L}$ " and "an $S$ is a $P_{S}$ " (where $P_{L}$ is the larger category of $S$ and $P_{S}$ is the smaller category) and production frequencies. However, the type of production frequency utilized was not specified. The resulting correlation was $r=.47(d f=24, p<.05)$ which is rather low. Clearly, the category size predictions of the feature-comparison model need more convincing evidence.

Evaluation of the Category Size Effect

Having reviewed the major findings regarding category size, it should be clear that the category size effect is a variable phenomenon and such evidence is not adequate to verify any particular model. More methodologically rigorous experiments are needed that will include a proper procedure for estimating category size. Category size has usually being manipulated by nested categories such as "word", "noun", 
"living thing", "animal" and "bird". Every category includes by definition everything that belonged in all smaller categories. Thus, it is assumed that "word" includes "noun", "living thing", "animal" and so on. Another commonly used manipulation of category size is by enumerating the instances of a category as they appear in a popular thesaurus. Neither procedure offers evidence on whether category size is indeed a psychological variable. One possible procedure would be to have subjects give lists of words that they can produce as members of a category. However, as Landauer and Meyer (1972) pointed out, we cannot be sure that the number of different members that a subject or group of subjects emit in a production task would be necessarily related to the number that they can recognize. Therefore, the most promising procedure is, as Johnson-Laird (1974) remarked, to utilize the number of items individual subjects actually recognize as members of a category. The Kintsch Model

A different approach to the structure of memory is offered by Kintsch $(1972,1974)$. His model is a psycholinguistic one that attempts to connect distinct meanings of words with sentence derivations. The model assumes that the basic units of meaning are propositions. Propositions are $n$-tuples of word concepts ( $n$-tuple is a set of $n$ elements where the order of elements is important) one of which serves as a predicator and the remaining cues as arguments, each fulfilling a unique semantic role. The predicator specifies a relationship among the arguments of a proposition. To use Kintsch's (1975) example, in the proposition (LOVE, Experiencer: GREEK, Object: ART) ${ }^{\top}$ there are two letters and enclosed in round brackets. 
arguments, GREEK and ART, and the predicator LOVE; in English this proposition could be realized with the sentence "the Greeks love art". The arguments of a proposition are concepts rather than words. Word concepts are stored in a person's semantic memory, which indicates the combinations of word concepts that form acceptable propositions. Word concepts are defined by the propositions in which they are used.

Kintsch used the linguistic theory of Fillmore $(1968,1969)$ to transform propositional expressions into English sentences. Though the lexical entries in the structure of lexical memory may be phrases, they are characterized by a list of propositions. The appropriate propositions are connected through the operations of conjunction $(\Lambda)$ and disjunction (V). For instance, suppose one wants to represent a person's concept of APPLE by means of the following combination of propositions: (FRUIT, APPLE)^(DELICIOUS, APPLE)^(RED, APPLE)V(YELLOW, APPLE)V (GREEN, APPLE). Conjunction has its usual meaning (and). Disjunction is to be understood as exclusive disjunction; that is, it asserts one, but not both, or the disjoint elements.

After discussing the structure of the set of all propositions, Kintsch presents a classification system for nouns and verbs. The structure of the noun system is a set-inclusion hierarchy that cannot be represented as a tree, because each element may have two immediate successors rather than a unique successor required for tree representation. Furthermore, it is not a lattice, because any pair of elements $A$ and $B$ have at least upper bound, but not a 11 pairs have greatest lower bounds. Furthermore, special classification rules of the following form are required to partition a category in more than one 
way:

$$
\left\{N_{1} V N_{2} V \ldots V N_{n}\right\} \rightarrow C
$$

where the $\mathrm{N}_{\mathrm{i}}$ 's are the sets of nouns that partition the category $\mathrm{C}$.

Kintsch describes the organization of the verb system as being more complex. While for the noun hierarchy nominal propositions are the building blocks of memory, for the verb structure there are all kinds of propositions. The structure of the verb system is again a partially-ordered set with a unique maximal element and many minimal ones. Similarly, it is not a lattice, because not all pairs of elements have greatest lower bounds. Verb-propositions participate in a large network of relationships merely by virtue of the fact that the nouns that they take as arguments are themselves members of various lexical structures. In addition, complex dependencies may exist among the arguments of a verb, so that a given verb may be characterized not just by its obligatory or deletable case, but by certain alternative patterns of cases (such as human or non-human object, or the object may be combined with an agent in some cases but not in others, etc.). Kintsch further considers that classification of propositions through the arguments they take is insufficient in iteself. Special rules to establish antonymous $n$-tuples are needed. These rules are of the form:

$$
\left[\left(v_{1}, x\right) v\left(v_{2}, x\right) \vee \ldots v\left(v_{n}, x\right)\right] \rightarrow(c, x)
$$

where $X$ is a noun (or more precisely, a class of nouns, since nouns in general possess various subordinate terms) and $(C, X)$. is a superordinate proposition. The set of propositions $\left(V_{1}, X\right) \vee \ldots V\left(V_{n}, X\right)$ forms a 
partition of $(C, X)$ in much the same way that the set of nouns forms a partition of a superordinate noun class.

Since propositions are a central assumption of this model, Kintsch (1972) attempted to obtain evidence concerning their status as basic lexical elements. He gave subjects simple sentences and asked them to tell if the sentences conveyed information not explicitly stated. Two types of sentences were selected. One type of sentence was incomplete, in the sense that cases that could and often would be part of such a sentence were omitted. An example of the first type sentence was "the secretary types" which could be completed with "on the paper" or "with a typewriter" etc. For the other type of sentences no additional cases could be part of these sentences, but for each sentence there were some more or less specific semantic implications. Such sentences were "it rains" which implies wet, clouds, water falling, etc.

The results showed that there were more idiosyncratic responses $\left(\bar{X}_{1}=4.4\right)$ than common responses $\left.\bar{x}_{2}=3.2\right)$. That is, al though no single response occurred with high frequency, for every sentence one or more dominating responses existed. Based on these results, Kintsch concluded that propositions are the building blocks of memory. However, to the present author it seems obvious that if "missing" sentences or sentences with many semantic implications have a dominant response, this only signifies that we speak in propositions and that we tend to fill in incomplete sentences when given the opportunity to do so. Furthermore, the dominant response was not overwhelmingly "dominant". Therefore, this evidence is not conclusive for Kintsch's model. 
Support for Kintsch's model was sought through an experiment conducted by Kintsch and Keenan (1973). They demonstrated that the number of propositions in a text base increased monotonically with reading time even if the length of the corresponding texts was controlled. However, this variable only accounted for $21 \%$ of the variance. In a later experiment Kintsch, Kozminsky, Streby, McKoon and Keenan (1975) found that reading times were longer and recall was less for texts with many different word concepts than for texts with fewer word concepts. The number of words and number of propositions were controlled. Superordinate propositions were recalled better than subordinate propositions and forgotten less when recall was delayed. The probability that a word concept was recalled increased as a function of both the number of repetitions of that concept in the text base and the number of repetitions of the corresponding word in the actual text. Whether propositions which contain word concepts are stored in a person's semantic memory is still questionable. Kintsch has shown that reading time is affected by number of propositions and word concepts, but such an effect could be due to an interaction of short-term memory and semantic memory. Presenting subjects with paragraphs and asking them to recall immediately involves both short-term memory and semantic retrieval. Further, whether reading time can be equated with semantic retrieval is questionable, if one takes into account the fact that the subject knows that he will be immediately tested for recall. The semantic retrieval is confounded with the fact that material is to be recalled and the subject could reread some parts of the paragraph but not others. Determining if propositions are the building blocks of 
memory requires more well-controlled experiments that involve only semantic retrieval. But even more important will be a clear delineation of his model from the existing psychological models; that is, a clear specification of what it means for propositions rather than a word or a concept to be stored. 
APPENDIX II

TABLE a

Stimuli Words

\begin{tabular}{|c|c|c|c|}
\hline Words & $\begin{array}{c}\text { Rosch's } \\
\text { Relatedness } \\
\text { Norms } \\
\end{array}$ & $\begin{array}{l}\text { Kǔcera- } \\
\text { Francis } \\
\text { Frequency }\end{array}$ & $\begin{array}{c}\text { Battig \& } \\
\text { Montague } \\
\text { Production } \\
\text { Frequency } \\
\end{array}$ \\
\hline Vegetable & -- & 10 & - \\
\hline $\begin{array}{l}\text { Spinach } \\
\text { Tomato } \\
\text { Parsley } \\
\text { Dandelion }\end{array}$ & $\begin{array}{l}1.22 \\
2.23 \\
3.32 \\
5.20\end{array}$ & $\begin{array}{l}2 \\
4 \\
1 \\
1\end{array}$ & $\begin{array}{r}163 \\
215 \\
15 \\
1\end{array}$ \\
\hline Fruit & -- & 35 & - \\
\hline $\begin{array}{l}\text { Plum } \\
\text { Watermelon } \\
\text { Gooseberry } \\
\text { Avocado }\end{array}$ & $\begin{array}{l}1.37 \\
2.39 \\
3.33 \\
5.37\end{array}$ & $\begin{array}{r}1 \\
1 \\
11\end{array}$ & $\begin{array}{r}167 \\
47 \\
1 \\
17\end{array}$ \\
\hline Bird & -- & 31 & - \\
\hline $\begin{array}{l}\text { Sparrow } \\
\text { Parrot } \\
\text { Vulture } \\
\text { Penguin }\end{array}$ & $\begin{array}{l}1.78 \\
2.07 \\
3.05 \\
4.53\end{array}$ & $\begin{array}{l}1 \\
1 \\
4 \\
-\end{array}$ & $\begin{array}{r}237 \\
72 \\
44 \\
14\end{array}$ \\
\hline Furniture & -- & 39 & - \\
\hline $\begin{array}{l}\text { Table } \\
\text { Bookcase } \\
\text { Stool } \\
\text { Wastebasket }\end{array}$ & $\begin{array}{l}1.10 \\
2.15 \\
3.13 \\
5.34\end{array}$ & $\begin{array}{r}198 \\
2 \\
8 \\
2\end{array}$ & $\begin{array}{r}408 \\
43 \\
72 \\
1\end{array}$ \\
\hline Vehicle & -- & 35 & - \\
\hline $\begin{array}{l}\text { Car } \\
\text { Trolley } \\
\text { Scooter } \\
\text { Skates }\end{array}$ & $\begin{array}{l}1.24 \\
2.19 \\
3.24 \\
4.99\end{array}$ & $\begin{array}{r}274 \\
5 \\
- \\
1\end{array}$ & $\begin{array}{r}407 \\
30 \\
44 \\
10\end{array}$ \\
\hline
\end{tabular}




\section{BIBL IOGRAPHY}

1. Anderson, J. R. and Reder, L. M. Negative judgments in and about semantic memory. Journal of Verbal Learning and Verbal Behavior, 1974, 13, 664-681.

2. Battig, W. F. and Montague, W. E. Category norms for verbal items in 56 categories: A replication and extension of the Connecticut norms. Journal of Experimental Psychology Monograph, 1969 , 80(3) Pt. 2.

3. Bierwisch, M. On classifying semantic features. In D. D. Steinberg and L. A. Jakobovits (Eds.) Semantics: An Interdisciplinary Reader in Philosophy, Linguistics and Psychology, Cambridge, England: University Press, 1971.

4. Bousfield, W. A. The occurrence of clustering in the recall of randomly arranged associates. Journal of General Psychology, $1953,49,229-240$.

5. Brown, R. W. and D. McNei11. The "tip-of-the-tongue" phenomenon. Journal of Verbal Learning and Verbal Behavior, 1966, $\underline{5}$, 325-337.

6. Clark, H. H. Word Associations and Linguistic Theory. In J. Lyons (Ed.) New Horizons in Linguistics. Baltimore: Penguin, 1970.

7. Cofer, C. N. and Musgrave, B. S. (Eds.) Verbal Behavior and Learning: Problems and Processes. New York: McGraw-Hi11 Book Company, 1963.

8. Collins, A. M. and Quillian, M. R. Retrieval time for semantic memory. Journal of Verbal Learning and Verbal Behavior, 1969, $\underline{8}, 240-24 \overline{8}$.

9. Collins, A. M. and Quillian, M. R. Facilitating retrieval from semantic memory: The effect of repeating part of an inference. Acta Psychologica, 1970a, 3, 304-314.

10. Collins, A. M. and Quillian, M. R. Experiments on semantic memory and language comprehension. In L.W. Gregg (Ed.) Cognition in Learning and Memory. New York: Wiley, 1970b.

11. Collins, A. M. and Quillian, M. R. Categories and semantic subcategories in semantic memory. Paper presented at the annual meeting of Psychonomic Society, St. Louis, M0, 1971.

12. Conrad, C. An analysis of a hierarchial model of semantic memory 
organization. Unpublished master's thesis, University of Oregon, 1971.

13. Conrad, C. Cognitive economy in semantic memory. Journal of Experimental Psychology, 1972, 92, 149-154.

14. Deese, J. The structure of associations in language and thought. Baltimore: Johns Hopkins Press.

15. Deese, J. On the structure of associative meaning. Psychological Review, 1962, 69, 161-175.

16. Fillenbaum, S. and Rapoport, A. Structure in subjective lexicon. New York: Academic Press, 1971.

17. Fillmore, C. J. The case for case. In E. Bach and R. T. Harms (Eds.) Universals in linguistic theory. New York: Holt, Rinehart and Winston, 1968, pp. 1-90.

18. Fillmore, C. J. Types of lexical information. In F. Kiefer (Ed.) Studies in syntax and semantics. Dodrecht, Holland: Reidel, 1969, pp.109-137.

19. Freedman, J. L. and Loftus, E. F. Retrieval of words from longterm memory. Journal of Verbal Learning and Verbal Behavior, $1971,10,107-115$.

20. Freedman, J. L. and Loftus, E. F. Retrieval of words from welllearned sets: The effect of category size. Journal of Experimental Psychology, 1974, 102, 1085-1091.

21. Glass, A. L. and Holyoak, K. J. The effect of "some" and "all" on reaction time for semantic decisions. Memory and Cognition, $1974, \underline{2}, 413-416$.

22. Glass, A. L. and Holyoak, K. J. Alternative Conceptions of Semantic Memory. Cognition, 1974/1975, 3(4), p.313-319.

23. Glass, A. L., Holyoak, K. J. and 0'Dell, C. Production frequency and the verification of quantified statements. Journal of Verbal Learning and Verbal Behavior, 1969,8 , 176-184.

24. Grober, E. and Loftus, E. F. Semantic memory: Searching for attributes versus searching for names. Memory and Cognition, $1974,2,413-416$.

25. Holyoak, K. J. and Glass, A. L. The role of contradictions and counter-examples in the rejection of false sentences. Journal of Verbal Learning and Verbal Behavior, 1975, 14, 215-254.

26. Homa, D. Organization and long-term memory search. Memory and 
Cognition, 1973, I, 369-379.

27. Jenkins, J. J. and Palermo, D. S. Mediation processes and the acquisition of linguistic structure. in U. Bellugi and R. Brown (Eds.) The Acquisition of Language. Monographs of the Society for Research in Child Deyelopment, 1964, 29, (1), 147169.

28. Johnson-Laird, P. N. Experimental psycholinguistics. Annual Review of Psychology, 1974, 25, 135-160.

29. Katz, J. J. Semantic Theory. New York: Harper and Row, 1972.

30. Kintsch, $w$. Notes on the structure of meaning in memory. In Tulving, E. and Donaldson, W. (Eds.) Organization of Memory, New York and London: Academic Press, 1972.

31. Kintsch, $W$. The representation of meaning in memory, Hillsdale, NJ: L. E. Eribaum Associates, 1974.

32. Kintsch, W. and Keenan, J. M. Reading rate as a function of the number of propositions in the base structure of sentences. Cognitive Psychology, 1973, 5, 257-274.

33. Kintsch, W., Kozminsky, W. J., Streby, W. J., McKoon, G. and Keenan, J. M. Comprehension and recall of text as a function of content variables. Journal of Verbal Learning and Verbal Behavior, $1975,14,196-214$.

34. Lakoff, G. Hedges: A study in meaning criteria and the logic of fuzzy concepts. Papers from the eighth regional meeting, Chicago Linguistic Society, Chicago: University of Chicago Linguistics Department, 1972.

35. Landauer, T. K. and Freedman, J. L. Information retrieval from long-term memory: Category size and recognition time. Journal of Verbal Learning and Verbal Behavior, 1968, 7, 291295.

36. Landauer, T. K. and Meyer, D. E. Category size and semantic memory retrieval. Journal of Verbal Learning and Verbal Behavior, $1972,11,529-549$.

37. Loftus, E. F. Category dominance, instance dominance and categorization time, Journal of Experimental Psychology, 1972, 97, $70-74$.

38. Loftus, E. F. and Bolton, M. Retrieval of superordinates and subordinates. Journal of Experimental Psychology, 1974, 102, 121-124. 
39. Loftus, E. F. and Cole, W. Retrieval attribute and name information from semantic memory. Journal of Experimental Psychology, 1974, 102, 1116-1122.

40. Loftus, E. F. and Freedman, J. L. Effects of category name frequency on the speed of naming an instance of the category. Journal of Verbal Learning and Verbal Behavior, 1972, 11, 343347.

41. Loftus, E. F., Freedman, J. L. and Loftus, G. R. Retrieval of words from subordinate and superordinate categories in semantic hierarchies. Psychonomic Science, 1970, 21, 235-236.

42. Loftus, E. F. and Scheff, R. W. Categorization norms for 50 representative instances. Journal of Experimental Psychology, 1971, 91, 355-364.

43. Loftus, E. F. and Suppes, P. Structural variables that determine the speed of retrieving words from long-term memory. Journal of Verbal Learning and Verbal Behavior, 1972, 11, 770-777.

44. Meyer, D. E. On the representation and retrieval of stored semantic information. Cognitive Psychology, 1970, 1, 242-300.

45. Meyer, D. E. Correlated operations in searching stored semantic categories. Journal of Experimental Psychology, 1973, 99, 124-133.

46. Miller, G. A. A psychological method to investigate verbal concepts. Journal of Mathematical Psychology, 1969, $\underline{6}, 169-191$.

47. Miller, G. A. English verbs of motion: A case study in semantic and lexical memory. In A. W. Melton and E. Martin (Eds.) Coding processes in human memory, Washington, DC: Winston, 1972.

48. Osgood, C. E. The nature and measurement of meaning. Psychological Bulletin, 1952, 49, 197-237.

49. Quillian, M. R. Word concepts: A theory and simulation of some basic semantic capabilities. Behavioral Science, 1967, 12, 410-430.

50. Rips, L. J., Shoben, E. J., and Smith, E. E. Semantic distance and the verification of semantic relations. Journal of Verbal Learning and Verbal Behavior, 1973, 12, 1-20.

51. Rosch, E. On the internal structure of perceptual and semantic cateogires. In T. E. Moore (Ed.) Cognitive development and the acquisition of language. New York: Academic Press, 1973. 
52. Rosch, E. Cognitive representations of semantic categories. Journal of Experimental Psychology: General, 1975, 104, 192233.

53. Rosch, E. Linguistic relativity. In A. Silverstein (Ed.) Human Communication: Theoretical Perspectives. New York: HaTsted Press, 1974.

54. Sanford, A. J. and Seymour, P. H. K. The influence of response compatibility on a semantic classification task. Acta Psychologica, 1974, 38, 405-412.

55. Sapir, E. Grading: A study in semantics. Philosophy of Science, $1944,11,93-116$.

56. Schaeffer, B. and Wallace, R. The comparison of word meanings. Journal of Experimental Psychology, 1970, 86, 144-152.

57. Smith, E. E. Effects of familiarity on stimulus recognition and categorization. Journal of Experimental Psychology, 1967, 74, 324-332.

58. Smith, E. E. and Haviland, S. E., Buckley, P. B. and Sack, M. Retrieval of artificial facts from long-term memory. Journal of Verbal Learning and Verbal Behavior, 1972, 11, 583-593.

59. Smith, E. E., Shoben, E. J. and Rips, L. J. Structure and process in semantic memory: A featural model for semantic decisions. Psychological Review, 1974, 81, 214-241.

60. Tulving, E. Episodic and semantic memory. In E. Tulving and $W$. Donaldson (Eds.) Organization of Memory. New York: Academic Press, 1972.

61. Wilkins, A. T. Conjoint frequency, category size, and categorization time. Journal of Verbal Learning and Verbal Behavior, $1971,10,382-385$. 